Pontifícia Universidade $C_{\text {atólica }}$

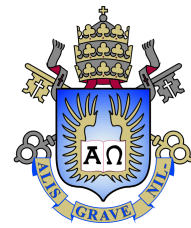

Fernando Abreu Rocha de Souza

\title{
The Clockwork Approach to Natural Fermion Hierarchies
}

Dissertação de Mestrado

Dissertation presented to the Programa de Pós-graduação em Física of PUC-Rio in partial fulfillment of the requirements for the degree of Mestre em Ciências - Física.

Advisor: Prof. Gero Arthur Hubertus Thilo Freiherr Von Gersdorff 


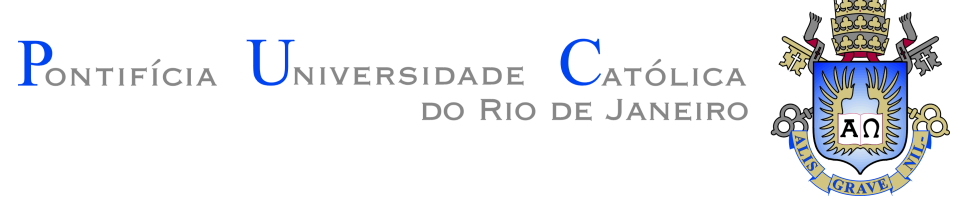

Fernando Abreu Rocha de Souza

\section{The Clockwork Approach to Natural Fermion \\ Hierarchies}

Dissertation presented to the Programa de Pós-graduação em Física of PUC-Rio in partial fulfillment of the requirements for the degree of Mestre em Ciências - Física. Approved by the Examination Committee.

Prof. Gero Arthur Hubertus Thilo Freiherr Von Gersdorff

Advisor

Departamento de Física - PUC-Rio

Prof. Rogerio Rosenfeld

Universidade Estadual Paulista Júlio de Mesquita Filho

Prof. Hiroshi Nunokawa

Departamento de Física - PUC-Rio

Rio de Janeiro, April 5th, 2019 
All rights reserved.

\section{Fernando Abreu Rocha de Souza}

The author graduated in Civil Engineering from Universidade Federal do Rio de Janeiro in 2016.

Bibliographic data

Abreu Rocha de Souza, Fernando

The Clockwork Approach to Natural Fermion Hierarchies / Fernando Abreu Rocha de Souza; advisor: Gero Arthur Hubertus Thilo Freiherr Von Gersdorff. - Rio de janeiro: PUCRio, Departamento de Física, 2019.

v., 113 f: il. color. ; $30 \mathrm{~cm}$

Dissertação (mestrado) - Pontifícia Universidade Católica do Rio de Janeiro, Departamento de Física.

Inclui bibliografia

1. Física - Teses. 2. Modelo Padrão - Teses. 3. Modelo Padrão;. 4. Física Além do Modelo Padrão;. 5. Hierarquia de Férmions;. 6. Física de Sabores;. 7. Mecanismo Clockwork.. I. Freiherr Von Gersdorff, Gero Arthur Hubertus Thilo. II. Pontifícia Universidade Católica do Rio de Janeiro. Departamento de Física. III. Título. 


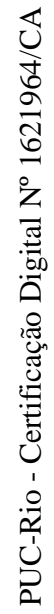

To my family and friends. 


\section{Acknowledgments}

I would like to thank my advisor for always being available for important weekly discussions and for patiently guiding me through the development of this work.

I would like to thank my parents and my grandparents for all the love and support.

I would like to thank my graduate professors for all the crucial teachings that have opened my eyes to the fascinating world of particle physics.

I would like to thank the funding agencies CNPq and CAPES. I am very grateful for the provided financial support.

This study was financed in part by the Coordenação de Aperfeiçoamento de Pessoal de Nível Superior - Brasil (CAPES) - Finance Code 001.

Finally, I would like to give a special thank to Lorenna for all the encouragement and for always believing in my potential. I would not be able to pursuit a career in Physics without your constant incentive and for that, I am eternally grateful. 


\section{Abstract}

Abreu Rocha de Souza, Fernando; Freiherr Von Gersdorff, Gero Arthur Hubertus Thilo (Advisor). The Clockwork Approach to Natural Fermion Hierarchies. Rio de Janeiro, 2019. 113p. Dissertação de mestrado - Departamento de Física, Pontifícia Universidade Católica do Rio de Janeiro.

The Standard Model of particle physics is one of the most well established theories in the field of physics and is able to make predictions correctly measured and verified up to twelve significant figures. However, the theory leaves some unanswered questions that have been bothering physicist for many years. One of those questions is the hierarchical structure of the fermion sector, where Yukawa matrices have eigenvalues that differ from each other by several orders of magnitude. Another aspect concerns the CKM matrix, which dictates the mixing between fermions of distinct flavours: why is this matrix almost diagonal, and why are the mixing angles so small? Why is the electron so much lighter than its cousins from different generations? The same question could be made for the quarks and the Standard Model would not be able to answer neither of these. In this work, an explanation is proposed by employing a novel model, called Clockwork Mechanism, which assumes the existence of new heavy fermion particles, named Clockwork Gears, which are able to naturally generate exponentially suppressed couplings out of order-one Yukawas, after spontaneously symmetry breaking occurs. In addition, simulations were run in order to optimize the free parameters of the model, as well as to confirm its efficiency at fitting with experimental data. Lastly, a few processes involving Flavour Changing Neutral Currents were considered in the effective field theory regime as a means to stipulate a typical mass scale for these new particles.

\section{Keywords}

Standard Model; Physics Beyond the Standard Model; ; Fermion Hierarchies; Flavour Physics; Clockwork Mechanism. 


\section{Resumo}

Abreu Rocha de Souza, Fernando; Freiherr Von Gersdorff, Gero Arthur Hubertus Thilo. O formalismo Clockwork para Hierarquias Naturais de Férmions. Rio de Janeiro, 2019. 113p. Dissertação de Mestrado - Departamento de Física, Pontifícia Universidade Católica do Rio de Janeiro.

O Modelo Padrão de física de partículas é uma das teorias mais bem estabelecidas no campo da física, sendo capaz de fazer previsões verificadas experimentalmente até doze algoritmos significativos. No entanto, o Modelo deixa algumas perguntas sem resposta, o que vem perturbando os físicos por muitos anos. Uma dessas questões é a estrutura hierárquica presente no setor dos férmions, onde matrizes Yukawas possuem autovalores que diferem um do outro por várias ordens de magnitude. Outro aspecto cabível de investigação é relacionado com a matriz CKM, responsável pela mistura entre férmions de sabores distintos. Por que tal matriz é aproximadamente diagonal e por que os ângulos de mistura são tão pequenos? Por que o elétron é muito mais leve que seus primos de outras gerações? A mesma pergunta pode ser feita para os quarks e o Modelo Padrão não seria capaz de responder nenhuma delas. Nesse trabalho, uma explicação proposta vem da utilização de um novo modelo, chamado de Mecanismo Clockwork, que pressupõe a existência de novos férmions pesados, nomeados Clockwork Gears, que são capazes de naturalmente gerar acoplamentos exponencialmente suprimidos a partir de Yukawas de ordem um, após a ocorrência de quebra espontânea de simetria. Além disso, simulações foram feitas com o objetivo de otimizar os parâmetros livres do modelo, assim como confirmar sua eficiência em acomodar os dados experimentais. Por fim, foi feita uma análise de alguns processos, envolvendo correntes neutras que trocam sabor, no regime de teoria efetiva de campo, para poder-se estipular um limite para a escala típica de massa para essas novas partículas.

\section{Palavras-chave}

Modelo Padrão; Física Além do Modelo Padrão; Hierarquia de Férmions; Física de Sabores; Mecanismo Clockwork. 


\section{Table of contents}

$\begin{array}{llr}1 & \text { Introduction } & 15\end{array}$

2 The Standard Model $\quad 20$

2.1 Spontaneous symmetry breaking 20

2.1.1 The linear sigma model $\quad 22$

2.1.2 The Higgs mechanism 24

2.2 Weak interactions 26

$\begin{array}{ll}2.2 .1 & \text { The gauge sector } \\ 2.2 .2 & 27\end{array}$

$\begin{array}{ll}2.2 .2 & \text { The fermion sector }\end{array}$

2.2.2.1 The quark sector 31

2.2.2.2 The lepton sector and the see-saw mechanism 35

2.3 Simulations for the Standard Model free parameters in the fermion sector 37

2.4 The path that leads to physics beyond the Standard Model 44

$3 \quad$ Froggatt-Nielsen mechanism for mass hierarchy 48

3.1 Two family model 49

3.2 Three family model $\quad 52$

3.2.1 Simulations for the three family model 53

4 Clockwork Model $\quad 58$

4.1 Clockwork fermion $\quad 59$

4.2 Hierarchy from product of random matrices 67

5 Simulations $\quad 73$

5.1 The clockwork mechanism applied to the Standard Model 73

5.2 Computational analysis $\quad 76$

5.2.1 Assumptions and procedures $\quad 76$

$\begin{array}{ll}\text { 5.2.2 Results for the quark sector } & 78\end{array}$

5.2.3 Results for the lepton sector 82

$6 \quad$ Flavour changing neutral currents $\quad \mathbf{8 6}$

$\begin{array}{lll}6.1 & \text { Building an effective field theory } & 86\end{array}$

6.2 The $\mathcal{L}_{6}$ operators $\quad 92$

6.2.1 $\mu \rightarrow e e e \quad 92$

6.2.2 $\mu \rightarrow e \gamma \quad 94$

$\begin{array}{ll}6.2 .3 \quad b \rightarrow s \gamma & 95\end{array}$

6.2.4 $K-\bar{K}$ mixing $\quad 96$

6.2.5 Choosing the right process for computational analysis 97

6.3 Muon decay 97

$\begin{array}{ll}\text { 6.3.1 Muon decay simulation } & 100\end{array}$

7 Conclusion 105

$\begin{array}{ll}\text { Bibliography } & 107\end{array}$ 


\section{List of figures}

Figure 1.1 Feynman diagram for the 1-loop correction for the electron mass in QED. The external momenta are set to zero to exclude the kinetic term. The fermion propagator contains the electron mass $m_{e}$ causing the whole diagram to be proportional to $m_{e}$ after one integrates over $q$.

Figure 2.1 Plots for the potential $V(\phi)$ from Eq. (2-1) for the two possible situations: (a) $\mu^{2}<0$, there is only one minimum value for the potential and (b) $\mu^{2}>0$, there are two minimum values for the potential and the vacuum state is degenerated.

Figure 2.2 Potential for two scalar fields, $\phi_{1}$ and $\phi_{2}$ (often called mexican hat potential). The minima of the potential are contained in a circumference (dashed line), where oscillations around the tangential directions are described by the massless fields $\pi$ (Goldstone bosons).

Figure 2.3 Best fit values of the Higgs boson coupling to different particles as a function of particle mass for the combination of ATLAS and CMS data (28) .

Figure 2.4 Uniform distribution of the entries in the Yukawa matrices. The distribution is contained within a region in the shape of a square with side 1 . This distribution garantees that the simulation will reproduce a natural configuration containing only $\mathcal{O}(1)$ Yukawas.

Figure 2.5 Distributions for the quark masses under the SM, while considering $\mathcal{O}(1)$ Yukawas. Here it becomes clear how such measured experimental values for the down sector masses are very unlikely to randomly be generated under the SM configuration.

Figure 2.6 Distributions for the quark mixing parameters under the SM, while considering $\mathcal{O}(1)$ Yukawas. Here it is clear that the small nature of the CKM mixing angles are very hard to come by according to the distribution for the SM

Figure 2.7 Distributions for the lepton masses under the SM, while considering $\mathcal{O}(1)$ Yukawas. Here it is clear how the charged lepton masses are very unlikely to randomly fall near the experimental lines for the SM configuration.

Figure 2.8 Distributions for the lepton mixing parameters under the $\mathrm{SM}$, while considering $\mathcal{O}(1)$ Yukawas. Here, since $\theta_{13}$ is the only small angle, its experimental line ends up falling way off on the distribution curve.

Figure 2.9 Feynman diagrams for the muon decay as given by: (a) The SM electroweak theory, where the muon decay is mediated by the $W$ boson and (b) Low energy regime, where the $W$ propagator can be broken down to a single vertex and constitute a dimension- 6 four-fermion interaction. 
Figure 3.1 Quark masses distributions for the Froggatt-Nielsen mechanism. One can clearly see that the distributions match perfectly with the experimental values, when the optimized value of $n=2$ is used.

Figure 3.2 Quark mixing distributions for the Froggatt-Nielsen mechanism. The experimental values seem to fit greatly within the distributions. As it was expected, from Eq. (3-36), the distributions for $\theta_{12}$ and $\theta_{23}$ are very similar.

Figure 4.1 Lattice configuration of the clockwork mechanism for fermions. Here the parameters $m$ and $k$ are taken as universal in order to simplify the model.

Figure 4.2 Distribution of the eigenvalues of product of random matrices. One can undoubtly see a hierarchical distribution for the eigenvalues.

Figure 4.3 Distribution of the eigenvalues of $Q^{\dagger^{N}} Q^{N}$. Once again, a hierarchical structure is clearly manifest under the distribution of the eigenvalues.

Figure 4.4 Distribution of the eigenvalues of $Z$. In this case, the mid eigenvalue seems to be closer to the greater one than to the smaller one. This behaviour agrees with Eq. (4-29).

Figure 4.5 Distribution of the eigenvalues of $Z^{-1 / 2}$. The hieararchical structure presented here is the responsible for generating the flavour hierarchy in the Clockwork fermion model.

Figure 5.1 Quark masses distributions for $N_{q}=9, N_{u}=9, N_{d}=6$, $c_{u}=1.70$ and $c_{d}=0.03$. The experimental values fall well within the reach of the distributions, especially for $y_{t}$ and $y_{b}$.

Figure 5.2 Quark mixing distributions for $N_{q}=9, N_{u}=9, N_{d}=6$, $c_{u}=1.70$ and $c_{d}=0.03$. The experimentally measured values fit greatly with the distributions obtained.

Figure 5.3 Lepton masses distributions for $N_{l}=2, N_{e}=9, N_{\nu}=0$, $c_{d}=0.02$ and $m_{R}=4.26 \times 10^{14} \mathrm{GeV}$. The experimental values fall well within the reach of the distributions.

Figure 5.4 Lepton mixing distributions for $N_{l}=2, N_{e}=9, N_{\nu}=0$, $c_{d}=0.02$ and $m_{R}=4.26 \times 10^{14} \mathrm{GeV}$. The experimentally measured values fit greatly with the distributions obtained.

Figure 6.1 Feynman diagram for $\mu \rightarrow$ eee. This a diagram is composed by a dimension- 6 and dimension- 4 vertices.

Figure 6.2 Feynman diagram for $\mu \rightarrow e \gamma$. This diagram is heavily suppressed by the loop correction factor.

Figure 6.3 Feynman diagram for $b \rightarrow s \gamma$. This decay mode is heavily suppressed due to the 1-loop quantum corrections.

Figure 6.4 Feynman diagram for $K-\bar{K}$ mixing. This diagram is build from the insertion of two dimension- 6 operators which configurates two flavour changings $(\Delta F=2)$.

Figure 6.5 Feynman diagrams for the muon decay. There are two possible diagrams. 
Figure 6.6 EFT Feynman diagram. Here the $Z$ propagator was broken down to a single 4-fermion interaction vertex

Figure 6.7 BR distributions. Here the parameter $\epsilon^{4}$ is responsible for shifting the distribution to the point where $95 \%$ of the values are below the experimental value for the BR.

Figure 6.8 Distributions for $B R^{\prime}$ and $\left|\Delta_{12}^{i}\right|^{2}$. Here it becomes clear that $B R^{\prime}$ is heavily dependent on $\left|\Delta_{12}^{l}\right|^{2}$ and much less to $\left|\Delta_{12}^{e}\right|^{2} .104$

Figure 6.9 Correlation between $\frac{m_{e}}{m_{\mu}}$ and BR. Here one can see a small correlation between them. 


\section{List of tables}

Table 2.1 Hypercharge and multiplicity of fields under SU(2). The notation here can be clarified by the example $2_{-\frac{1}{2}}$, which means that the field transforms as a doublet under $S U(2)$ and has hypercharge $-\frac{1}{2}$.

Table 2.2 Experimentally measured values for the quark masses (7). The hierarchy across families and between the up and down sector is clear. The experimental errors involved are ommited due to the fact that their contribution is neglectable for the development of the present work.

Table 2.3 Experimentally measured values for the mixing angles and the Jarlskog invariant in the quark sector (7). The mixing angles are clearly very small, and the CKM matrix can be taken as almost diagonal. The experimental errors involved are ommited due to the fact that their contribution is neglectable for the development of the present work.

Table 2.4 Experimentally measured values for the mixing angles and the $J_{C P}$ invariant in the lepton sector (7). The mixing angles are not small, and the CKM matrix can not be taken as almost diagonal. Here, normal ordering was considered and $\theta_{23}$ was taken in the first octant. The experimental errors involved are ommited due to the fact that their contribution is neglectable for the development of the present work.

Table 2.5 Experimentally measured values for the lepton masses (7). The hierarchy across families and between the up sector and the charged leptons is clear (see Table 2.2 for comparison). Note that, at the time of this work, neutrino masses have not yet been measured, only the difference between squared masses have been experimentally verified, that is $\Delta m_{i j}^{2} \equiv\left|m_{i}^{2}-m_{j}^{2}\right|$. The experimental errors involved are ommited due to the fact that their contribution is neglectable for the development of the present work.

Table 2.6 Best fit free parameters for the SM distribution, in order to attempt to account for the hierarchical structure of fermion masses. The values computed for $\chi_{\min }^{2}$ are way too high, which can be interpreted as a extremely low likelihood of obtaining the experimental values from the simulated distributions.

Table 2.7 Operators that constitute building blocks for theoretical models and their related dimensions in powers of $\hbar$ and length $L$ (46), (47), (48), (49). Here $M, g, \phi, A_{\mu}$ and $\psi$ are a mass scale, a coupling, a scalar field, a vector field and a spinor field, respectively. 
Table 3.1 Quarks charges assigned for the $U(1)$ flavor symmetry (50). Here, the parameter $n$ is introduced to allow an optimization of the mass hierarchy via computer simulation.

Table 3.2 Quark sector variables and their experimental values taken from (7).

Table 3.3 Quark sector best fit free parameters for the FroggattNielsen mechanism. The value for $\epsilon$ corresponds to the initial expectation, as seen in (50), while the low value for $\chi_{\min }^{2}$ (taking into account the six degrees of freedom considered) is clear evidence for the fact that the model agrees very well with the experimental data.

Table 4.1 Charges for the clockwork fermion under different symmetries. The charges are assigned in such a way that one can build invariant terms as $m_{j} \bar{\psi}_{L}^{j} \psi_{R}^{j}$ and $k_{j} \bar{\psi}_{L}^{j} \psi_{R}^{j+1}$.

Table 4.2 Zero mode localization for $\psi_{R}^{1}$ interpolating field. The localization of the zero mode and the strength of the coupling to $\psi_{R}^{1}$ are related to the magnitude of the spurion $q$.

Table 5.1 Vector-like clockwork fermions introduced. Apart from the neutrinos, there are two chiral fields introduced for each SM field.

Table 5.2 Quark sector variables and their experimental values (7). Once again, the experimental uncertainties were not taken into account as their contribution are neglectable.

Table 5.3 Quark sector best fit free parameters obtained through simulations. The values for $c_{u}$ and $c_{d}$ agrees with the prediction from Eq. (5-21), while the small value for $\chi_{\min }^{2}$ indicates that the distributions obtained match the experimental data. The 10 degrees are freedom involved in the computation of $\chi_{\min }^{2}$ are the ones related to the parameters presented in Table 5.2.

Table 5.4 Lepton sector variables and their experimental values (7). Once again, the experimental uncertainties were not taken into account as their contribution are neglectable.

Table 5.5 Lepton sector best fit free parameters obtained through simulations. The values for $c_{e}$ agrees with the prediction from Eq. (5-21), while the small value for $\chi_{\min }^{2}$ indicates that the distributions obtained match the experimental data. The 9 degrees are freedom involved in the computation of $\chi_{\min }^{2}$ are the ones related to the parameters presented in Table 5.2.

Table 6.1 FCNC processes in the effective Clockwork model. $\quad 92$

Table 6.2 Upper bounds for FCNC Branching Ratios.

Table 7.1 Summarized results for the quark sector best fit free parameters obtained through simulations.

Table 7.2 Summarized results for the lepton sector best fit free parameters obtained through simulations. 
At heart, science is the quest for awesome - the literal awe that you feel when you understand something profound for the first time. It's a feeling we are all born with, although it often gets lost as we grow up and more mundane concerns take over our lives.

Sean Carroll, The Particle at the End of the Universe: How the Hunt for the Higgs Boson Leads Us to the Edge of a New World. 


\section{Introduction}

The Standard Model (SM) has been repeatedly proving, in the last couple of decades, to be an astounding theory that can be considered the most successful theoretical ground available for particle physicists. its triumph lies in a series of predictions that have been confirmed by precise experiments (1), which ultimately led to the detection of the Higgs boson (2). This vast aggregation of evidence confirming its validity allows one to reliably assure that, up to an energy scale, all the effective underlying physical structure which guides the behaviour of the particles around us, is well accounted for by the SM.

However, in spite of all the predictive power of the model and its overall success in describing the particles and forces in our universe, there are still several uncharted territories to explore in the field of particle physics that remain unexplained by the SM. Among those, one can mention the existence of dark matter (3), a proper theory of quantum gravity (4), the Higgs hierarchy problem (5) and, the one that will be the main focus of the present work, the fermion hierarchy problem (6).

As it will be shown in greater depth later, the symmetries the SM is based upon allow the existence of a total of 17 free parameters (among those are the fermion masses and mixing angles, excluding neutrino masses and Majorana phases). These free parameters are not predicted in any way by the theory, but rather only determined by experimental measurements. This way, even before the collection of experimental data, one should naturally expect that these parameter assume values that are not extremely high nor extremely low, but instead within a reasonable range of the axis origin.

The problem arises from the fact that the fermions in the SM constitute a family (often called generation or flavour) and one interesting aspect that was not predicted by the mathematical structure that underlies the SM, but rather incorporated in the model after its experimental discovery, is that there are two additional families with particles that are identical as the ones from the first generation in every possible way (same quantum numbers), except for the fact that their masses are heavier by several orders of magnitude, that is

$$
m_{\psi_{1}} \ll m_{\psi_{2}} \ll m_{\psi_{3}},
$$


where $m_{\psi_{i}}$ is the mass of the $i$-th generation fermion $\psi_{i}$. The expression in Eq. (1-1) can be interpreted as a manifestation of a hierarchical structure between masses of fermions across different flavours and is the reason why the problem got its denomination. The explicit hierarchy can be seen in the following relations $(7)$ :

$$
\begin{aligned}
\frac{m_{c}}{m_{u}} & \sim 6 \times 10^{2}, & \frac{m_{t}}{m_{u}} & \sim 8 \times 10^{4}, \\
\frac{m_{s}}{m_{d}} & \sim 2 \times 10, & \frac{m_{b}}{m_{d}} & \sim 2 \times 10^{2}, \\
\frac{m_{\mu}}{m_{e}} & \sim 2 \times 10^{2}, & \frac{m_{\tau}}{m_{e}} & \sim 3 \times 10^{3},
\end{aligned}
$$

As one can see from Eq. (1-2), the ratio between the masses across different flavours seems to be surprisingly large and our initial expectation of reasonable natural values for the free parameters in the theory is proven wrong by a large margin.

Another important feature that comes from experimental data, and is also considered part of the fermion hierarchy problem, is that the CKM (Cabibbo-Kobayashi-Maskawa) matrix, which encapsulates the flavour changing processes in the quark sector, is measured to be almost diagonal, with very small mixing angles between different flavours, whereas the PMNS (Pontecorvo-Maki-Nakagawa-Sakata) mixing matrix in the neutrino sector contains reasonably large mixing angles, that is

$$
V_{C K M} \sim\left(\begin{array}{ccc}
1 & & \\
& 1 & \\
& & 1
\end{array}\right), \quad V_{P M N S} \sim\left(\begin{array}{ccc}
1 & 1 & 1 \\
1 & 1 & 1 \\
1 & 1 & 1
\end{array}\right) .
$$

However, here one might feel inclined to make the question as to why does this hierarchy between families and between mixing angles in different sectors is regarded as a problem? Can't we just accept that the universe is like that and be done with it? In order to be able to answer those questions, one must first uncover the meaning of naturalness in the context of theoretical physics.

The quest for naturalness, in the sense of mathematical elegance, which encapsulates criteria of simplicity and beauty, has always been a powerful guiding tool for physicists trying to build new theories and, being so, has exerted a strong influence in the field of particle physics. Under this scope, namely structural naturalness (8), the concept acquires a more subjective meaning and, even though can be viewed as a powerful compass pointing in right direction, it can not be solely used to validate a theory and, in fact, can 
sometimes be misleading due to the limited knowledge of the time. It is easy to see now why the heliocentric model is more natural than the geocentric one, as in the first one the motion of planets is described by simple elliptic orbits, while the latter requires a more complicated motion with epicyclic orbits within orbits. However, at the time the heliocentric model was postulated, it was most probably considered much less natural than the usual geocentric universe, due to philosophical and religious reasons.

A different facet of the natural criterion, deemed more precise and coined as numerical naturalness (8), was developed in the field of particle physics and has been playing a fundamental role in the formulation of theoretical predictions for new phenomena to be observed in the Large Hadron Collider (LHC) as well as in future particle accelerators. This criterion can be formulated in the following way: a free parameter in a theory is allowed to be much smaller than unity only if setting it to zero increases the symmetry of the theory (9). When this happens, one says that such small parameter is technically natural (10). This is precisely the case for the fermion hierarchy problem, as if one sets the Yukawas to zero in

$$
\begin{aligned}
\mathcal{L}=i \bar{q}_{L} \not D q_{L}+i \bar{d}_{R} \not D d_{R}+i \bar{u}_{R} \not D u_{R}+i \bar{l}_{L} \not D l_{L}+i \bar{e}_{R} \not D e_{R} \\
\\
+\bar{q}_{L} Y_{d} H d_{R}+\bar{q}_{L} Y_{u} \tilde{H} d_{R}+\bar{l}_{L} Y_{e} \tilde{H} e_{R}+\text { h.c. }
\end{aligned}
$$

which gives

$$
\mathcal{L}_{Y \rightarrow 0}=i \bar{q}_{L} \not D q_{L}+i \bar{d}_{R} \not D d_{R}+i \bar{u}_{R} \not D u_{R}+i \bar{l}_{L} \not D l_{L}+i \bar{e}_{R} \not D e_{R}
$$

and the SM Lagrangian acquires a flavour symmetry $[U(3)]^{5}$, as every matter field is now allowed to rotate freely between different flavours, while keeping the Lagrangian in Eq. (1-5) invariant, via the transformations

$$
q_{L} \rightarrow U_{q} q_{L}, \quad d_{R} \rightarrow U_{d} d_{R}, \quad u_{R} \rightarrow U_{u} u_{R}, \quad l_{L} \rightarrow U_{l} l_{L}, \quad e_{R} \rightarrow U_{e} e_{R}
$$

where $U$ is a $3 \times 3$ unitary matrix in flavour space. And, since even small Yukawas are still able to forbid the existence of a large $[S U(3)]^{5}$ flavour symmetry, these parameters are considered technically natural.

Another feature of the fermion masses is that their quantum corrections are proportional to themselves, this way if a fermion mass has a small value then the quantum corrections for such mass will be even smaller, and so such parameter will stably remain within very close range of its initial tiny value. This effect can be explained by taking the case of Quantum Electrodynamics (QED), where the electron mass term can be writen as

$$
\mathcal{L}_{\text {mass }}^{Q E D}=m_{e} \bar{e}_{L} e_{R}
$$


If one wishes to calculate the quantum corrections for the electron mass here, one should compute a loop diagram as in Fig. 1.1. Since we want a propagator to connect left-handed electrons to right-handed electrons, as in Eq. (1-7), and the photon propagator is chirality diagonal, the only possible solution is using a fermion propagator, which depends on the fermion mass, in this case the electron mass $m_{e}$, and so the diagram must be proportional to $m_{e}$, and, since the electron mass is known to be small, so are the related quantum corrections.

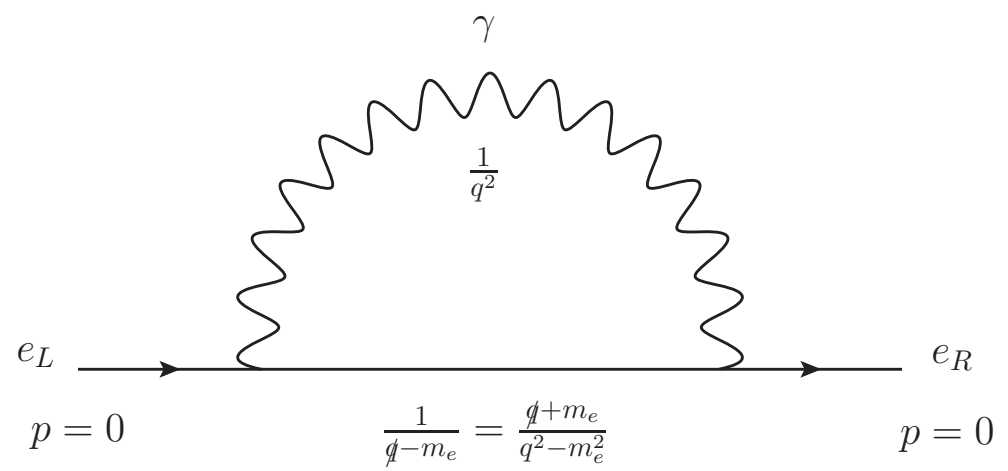

Figure 1.1: Feynman diagram for the 1-loop correction for the electron mass in QED. The external momenta are set to zero to exclude the kinetic term. The fermion propagator contains the electron mass $m_{e}$ causing the whole diagram to be proportional to $m_{e}$ after one integrates over $q$.

From this perspective, the fermion hierarchy problem is usually considered to be not as huge of a issue as the Higgs hierarchy (10), since the quantum corrections for the Higgs mass are proportional to the maximum energy scale available by virtual particles, yielding huge corrections to the former and a very high level of fine-tuning is necessary to make sense of the measured value for the mass of the Higgs boson $\left(m_{H}=125 \mathrm{GeV}\right.$ (2), while the corrections must be proportional to the energy scale $\Lambda \sim 10^{19} \mathrm{GeV}$ ).

Nonetheless, the hierarchy in the fermion sector is still a serious issue, since it seems rather suspicious that 17 arbitrary free parameters would be so fine-tuned in a way to produce such particular hierarchical spectra. Being so, is widely accepted in the literature (11) that this apparent fantastic numerical coincidence must have a hidden reason. Moreover, there is a reductionist belief among members of the theoretical physics community in the existence of an underlying theory of everything, under which all dimensionless parameters are determined and computable. By this view, seeking theories with fewer degrees of freedom seems to be a good guiding point for elaborating new models. In that sense, the model proposed in this thesis can be considered more natural and an improvement over the SM, as, it will be shown later, it requires the use of 8 free parameters, that are optimized via simulation, in addition to 15 parameters that can be regarded as random $\mathcal{O} 1$ complex numbers. 
Many models were developed as valid attempts to tackle this problem by giving possible natural explanations for such hierarchical phenomena. Here we investigate two interesting models, one is the well known Froggatt-Nielsen mechanism (6) while the other, which is the main subject of this thesis, is the novel clockwork mechanism (12), (13) and (14). The latter, as it will be shown in great detail later, revolves around a spontaneous symmetry breaking (SSB) of all but one symmetry in a lattice system with a symmetry $\mathcal{G}$ for each site. After SSB, one ends up with a single massless particle, called zero mode, which can be written as a linear combination of the former particles in the lattice, called clockwork gears. The strength of the model comes from the fact that the zero mode happens to be localised towards one of the boundaries, this way one could couple the SM particles to the opposite boundary and automatically yield strongly suppressed interactions.

When applied to the flavour hierarchy problem, the clockwork model allows to naturally obtain, out of order-one random complex matrices (15), fermion masses that follow a hierarchical structure. That is the main success of the mechanism, as it is able to naturally replicate, without any bold assumptions, a very specific flavour hierarchy found in nature.

This work is divided in the following fashion: In Chapter 2, a review of the Standard Model is presented, focusing on the fermion sector and the free parameters that it contains, while also providing a bridge to usual techniques used in Beyond the Standard Model (BSM) physics. Then, we proceed to the introduction of the Froggatt-Nielsen mechanism, in Chapter 3, presenting the simulated distribution for the fermion sector parameters in this mechanism. Chapter 4 uncovers the clockwork mechanism and its application to flavour hierarchy, while Chapter 5 exhibits the results of the computational simulations for the distributions of the free parameters in the fermion sector in the scope of the clockwork model. In Chapter 6, an analysis of the processes containing Flavour Changing Neutral Currents (FCNC), in the clockwork theory, is presented and a simulation is ran in order to estimate a typical mass scale for the clockwork gears. Finally, Chapter 7 gives a conclusion of the subject, judging the results obtained via simulation, and propose future related works. 


\section{2}

\section{The Standard Model}

The Standard Model (SM) is the result of decades of scientific efforts, via the accumulation of experimental evidences, in order to build a successful description of all the current known fundamental particles and forces (16). As it is well known across the scientific community, the mathematical tools that make such description possible comes from the development of Quantum Field Theory (QFT), which brings together the physics of Special Relativity and Quantum Mechanics. Under QFT, particles are considered to be fields in spacetime and among the different kind of fields possible in the theory, one can highlight the following: scalar fields $(\phi$, spin 0$)$, vector fields $\left(A_{\mu}\right.$, spin 1$)$ and spinor fields $\left(\psi\right.$, spin $\left.\frac{1}{2}\right)$.

An important observation that is encompassed by the SM, is that the fermion sector, which is composed by spinor fields, vector fields that mediate their interactions and a scalar field (Higgs) which gives rise to their masses, is divided into three generations (often called families or flavours) where the corresponding particles across the generations have identical charges and quantum numbers. Although not predicted by the SM, experimental evidence show that fermions across different families possess different masses that obey the following relation:

$$
m_{\psi_{1}} \ll m_{\psi_{2}} \ll m_{\psi_{3}},
$$

where $m_{\psi_{i}}$ is the mass of the $i$-th generation fermion $\psi_{i}$.

In this chapter, we present a brief review of the Standard Model with focus on the fermion sector (17), (18).

\section{1}

\section{Spontaneous symmetry breaking}

In order to understand how the matter particles acquire its mass, under the mathematical structure of QFT, one has to first comprehend how the Higgs mechanism works, in particular the concept of Spontaneous Symmetry Breaking (SSB).

SSB occurs when, even though a Lagrangian is invariant under a particular symmetry, the ground state of the theory happens to not be invariant under such symmetry. Let us see a simple example to better illustrate the point. The 
Lagrangian for a real scalar field $\phi$ is given by:

$$
\mathcal{L}_{\phi}=\frac{1}{2}\left(\partial_{\mu} \phi\right)\left(\partial^{\mu} \phi\right)-V(\phi), \quad \text { where } V(\phi)=-\frac{1}{2} \mu^{2} \phi^{2}+\frac{1}{4 !} \lambda \phi^{4},
$$

where a discrete transformation

$$
\phi \rightarrow-\phi
$$

is a global symmetry ( $\mathbb{Z}_{2}$ group) under this toy model. One can compute the expectation value of the vacuum by minimizing the potential $V$, which can be achieved by taking

$$
\frac{\partial}{\partial \phi} V(\phi)=0
$$

where the solution to Eq. (2-3) are the following:

$$
\begin{gathered}
\langle\phi\rangle_{\Omega} \equiv\langle\Omega|\phi| \Omega\rangle=0, \quad \text { if } \mu^{2}<0, \\
\langle\phi\rangle_{\Omega}= \pm \sqrt{\frac{6 \mu^{2}}{\lambda}}, \quad \text { if } \mu^{2}>0
\end{gathered}
$$

where $|\Omega\rangle$ is the vacuum state and both situations are illustrated in Fig. 2.1. Here one can see in Eq. (2-5) that, in the case where $\mu^{2}>0$, the vacuum state is degenerate, as it has two possible expectation values.

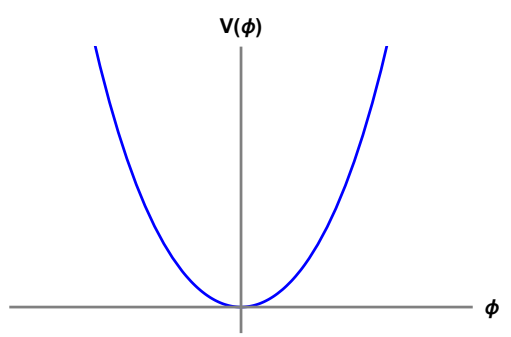

(a) $\mu^{2}<0, \quad\langle\phi\rangle_{\Omega}=0$

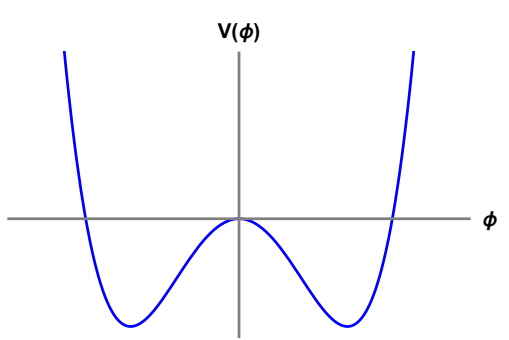

(b) $\mu^{2}>0, \quad\langle\phi\rangle_{\Omega}= \pm \sqrt{\frac{6 \mu^{2}}{\lambda}}$

Figure 2.1: Plots for the potential $V(\phi)$ from Eq. (2-1) for the two possible situations: (a) $\mu^{2}<0$, there is only one minimum value for the potential and (b) $\mu^{2}>0$, there are two minimum values for the potential and the vacuum state is degenerated.

An interesting result can be obtained when one performs an expansion of the scalar field around one of the minima (supposedly the positive one):

$$
\phi(x)=v+\sigma(x), \quad \text { where } v=\sqrt{\frac{6 \mu^{2}}{\lambda}}
$$

is the vacuum expectation value of $\phi$. Taking the expansion in Eq. (2-6) and plugging it in the Lagrangian in Eq. (2-1) allow us to rewrite the Lagrangian in terms of $\sigma(x)$ as 


$$
\mathcal{L}=\frac{1}{2}\left(\partial_{\mu} \sigma\right)\left(\partial^{\mu} \sigma\right)-\frac{1}{2}\left(2 \mu^{2}\right) \sigma^{2}-\sqrt{\frac{\lambda}{6}} \mu \sigma^{3}-\frac{1}{4 !} \sigma^{4},
$$

where the new Lagrangian, describing a scalar field with mass

$$
m_{\sigma}=\sqrt{2} \mu
$$

has picked up a cubic interaction $\sqrt{\frac{\lambda}{6}} \mu \sigma^{3}$ that was not present in the original Lagrangian, explicitly breaking the symmetry from Eq. (2-2). Therefore, due to the manifestation of two degenerate vacuum states, the expanded Lagrangian is no longer invariant under $\mathbb{Z}_{2}$. This is the most simple case of Spontaneous Symmetry Breaking, where the broken symmetry is a discrete one.

\subsection{1}

\section{The linear sigma model}

From here, one can investigate the case of a system with multiple scalar fields, as such Lagrangian will have continuous symmetry, rather than discrete ones, and a more interesting result is obtained. Here we take $N$ scalar fields and write the Lagrangian as:

$$
\mathcal{L}=\frac{1}{2}\left(\partial_{\mu} \phi^{i}\right)\left(\partial_{\mu} \phi^{i}\right)-V\left(\phi^{i}\right), \quad \text { where } V\left(\phi^{i}\right)=-\frac{1}{2} \mu^{2}\left(\phi^{i}\right)^{2}+\frac{1}{4} \lambda\left[\left(\phi^{i}\right)^{2}\right]^{2},
$$

and there is an implicit sum over $i$. This is called the linear sigma model. It is easy to see that the Lagrangian from Eq. (2-9) is invariant under the transformation

$$
\phi^{i} \rightarrow R_{i j} \phi^{j}
$$

where $R_{i j}$ can be any $N \times N$ orthogonal matrix (that is, $R^{T} R=\mathbb{1}$ ) and the set of all the orthogonal matrices forms the symmetry group $O(N)$, which describes a rotation in $N$ dimensions. Now, if one would minimize the potential $V$, while taking $\mu^{2}>0$, one would find that the vacuum state would also be degenerated, as any set of scalar fields $\phi^{i}$ that satisfies the relation

$$
\left(\phi_{0}^{i}\right)^{2}=\frac{\mu^{2}}{\lambda}
$$

would constitute a minimum energy state for the system. One can interpret Eq. (2-11) as a vector length in the scalar fields space and see that the vacua solutions form a sphere in a $N$-dimensional space, while the direction for such vector remains arbitrary. The simple case for $N=2$ can be seen in Fig. (2.2).

Without loss of generality, one can choose the solution to Eq. (2-11) to be the vector

$$
\phi_{0}^{i}=(0,0, \ldots, 0, v), \quad \text { where } v=\frac{\mu}{\sqrt{\lambda}}
$$

and, in order to access the behaviour of the system around the minima, perform an oscillation around the vacua by means of a shift in the vector coordinates 
in Eq. (2-12) by

$$
\phi^{i}(x)=\left(\pi^{1}(x), \pi^{2}(x), \ldots, \pi^{N-1}(x), v+\sigma(x)\right),
$$

where $\pi^{k}(x)(k=\{1, \ldots, N\})$ and $\sigma(x)$ are scalar fields that represent the aforementioned shift in the $k^{\text {th }}$ coordinate (The notation comes from the context of pions, as it was originally used). Now, take Eq. (2-13) and plug it into the Lagrangian in Eq. (2-9), rewriting it as

$$
\begin{aligned}
\mathcal{L}=\frac{1}{2}\left(\partial_{\mu} \pi^{k}\right)^{2}+\frac{1}{2} & \left(\partial_{\mu} \sigma\right)^{2}-\frac{1}{2}\left(2 \mu^{2}\right) \sigma^{2}-\sqrt{\lambda} \mu \sigma^{3} \\
& \quad-\sqrt{\lambda} \mu\left(\pi^{k}\right)^{2} \sigma-\frac{\lambda}{4} \sigma^{4}-\frac{\lambda}{2}\left(\pi^{k}\right)^{2} \sigma^{2}--\frac{\lambda}{4}\left[\left(\pi^{k}\right)^{2}\right]^{2} .
\end{aligned}
$$

Just like in Eq. (2-7), here we obtained a massive scalar field $\sigma$, while also acquiring a set of $N-1$ massless fields $\pi$. Moreover, it is easy to see that the original $O(N)$ symmetry is broken, while only a subgroup $O(N-1)$ survived the breaking. The appearance of these massless particles in the linear sigma model is a particular case of a more general result known as the Goldstone's theorem (19). The theorem states that for each continuous symmetry spontaneously broken, there must be a massless particle associated and these massless particles are called Goldstone bosons.

Let us count the number of symmetries broken by the linear sigma model to confirm that the theorem holds in this theory. We started with Eq. (2-9) which is invariant under $O(N)$, a rotation in $N$ dimensions. When performing a rotation in $N$ dimensional space, one has $N(N-1) / 2$ directions to choose from, which implies that the symmetry group $O(N)$ contains $N(N-1) / 2$ continuous symmetries. Whereas, in Eq. (2-14) the related symmetry group is $O(N-1)$ and so it contains $(N-1)(N-2) / 2$ continuous symmetries. The number of symmetries broken is simply the difference $N-1$, which is precisely the number of Goldstone bosons in the theory. 


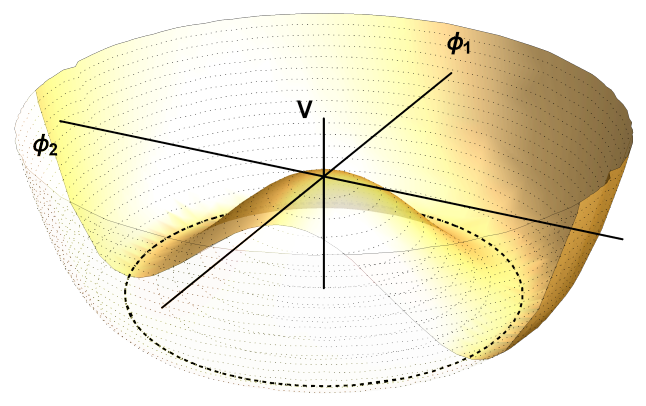

Figure 2.2: Potential for two scalar fields, $\phi_{1}$ and $\phi_{2}$ (often called mexican hat potential). The minima of the potential are contained in a circumference (dashed line), where oscillations around the tangential directions are described by the massless fields $\pi$ (Goldstone bosons).

\subsection{2}

\section{The Higgs mechanism}

Having seen how SSB works in practice, we are now ready to dive into the Higgs mechanism and see how vector fields and spinor fields acquire mass from SSB. Let us first introduce a masless vector field coupled to a complex scalar field in an Abelian theory and see how it affects the model. The corresponding Lagrangian is given by:

$$
\mathcal{L}=-\frac{1}{4} F^{\mu \nu} F_{\mu \nu}+\left|D_{\mu} \phi\right|^{2}-V(\phi),
$$

where $F_{\mu \nu}=\partial_{\nu} A_{\mu}-\partial_{\mu} A_{\nu}$ and $D_{\mu}=\partial_{\mu}+i g A_{\mu}$. The Lagrangian in Eq. (2-15) is locally invariant under the $U(1)$ group, which corresponds to the following transformations:

$$
\phi(x) \rightarrow e^{i \alpha(x)} \phi(x), \quad A_{\mu}(x) \rightarrow A_{\mu}(x)-\frac{1}{g} \partial_{\mu} \alpha(x) .
$$

Taking the potential to be

$$
V(\phi)=-\mu^{2}|\phi|^{2}+\frac{\lambda}{2}|\phi|^{4}
$$

where $\mu^{2}>0$, the $U(1)$ symmetry will be spontaneously broken by a vacuum expectation value of

$$
\langle\phi\rangle=\phi_{0}=\sqrt{\frac{\mu^{2}}{\lambda}}
$$

or any other value related by the rotation $e^{i \alpha(x)} \phi_{0}$. Once again, we proceed by making an expansion around the vacuum as

$$
\phi(x)=\phi_{0}+\frac{1}{\sqrt{2}}\left(\phi_{1}(x)+i \phi_{2}(x)\right)
$$

and plugging Eq. (2-19) in Eq. (2-17) gives 


$$
V(\phi)=-\frac{1}{2 \lambda} \mu^{4}+\frac{1}{2}\left(2 \mu^{2}\right) \phi_{1}^{2}+\mathcal{O}\left(\phi_{i}^{3}\right),
$$

where, once again, the $\phi_{1}$ acquired a $2 \mu^{2}$ mass while $\phi_{2}$ remained massless and is the Goldstone boson of the theory. However, when looking at the kinetic term

$$
\left|D_{\mu} \phi\right|^{2}=\frac{1}{2}\left(\partial_{\mu} \phi_{1}\right)+\frac{1}{2}\left(\partial_{\mu} \phi_{2}\right)+\frac{1}{2}\left(2 g^{2} \phi_{0}^{2}\right) A_{\mu} A^{\mu}+\ldots
$$

one can see that the gauge boson $A_{\mu}$ also acquired a mass term

$$
m_{A}=\sqrt{\frac{2}{\lambda}} g \mu .
$$

This mechanism, under which spontaneous symmetry breaking generates a mass for a gauge boson, was discovered almost simultaneously by different groups (20), (21), (22), (23), (24) and generalized for non-Abelian theories. This mechanism is mostly known as the Higgs mechanism.

One can make an extension of the mechanism to the non-Abelian case in a straightforward fashion. Here we will work out the most general case, and apply it to the weak interactions in the following section. Suppose a system with a set of scalar fields $\phi_{i}$ which is invariant under a symmetry $\mathcal{G}$, represented by the following transformation:

$$
\phi_{i} \rightarrow e^{\left(i \alpha^{a} t^{a}\right)_{i j}} \phi_{j} \approx\left(1+i \alpha^{a} t^{a}\right)_{i j} \phi_{j},
$$

where $t^{a}$ are the representation matrices of $\mathcal{G}$. The covariant derivative of the theory will be given by

$$
D_{\mu}=\partial_{\mu}-i g A_{\mu}^{a} t^{a}
$$

and the kinetic term can be written as

$$
\frac{1}{2}\left|D_{\mu} \phi_{i}\right|^{2}=\frac{1}{2}\left(\partial_{\mu} \phi_{i}\right)+i g A_{\mu}^{a}\left(\partial_{\mu} \phi_{i} t_{i j}^{a} \phi_{j}\right)-\frac{1}{2} g^{2} A_{\mu}^{a} A^{\mu b}\left(t^{a} \phi\right)_{i}\left(t^{b} \phi\right)_{j} .
$$

Now, letting $\phi_{i}$ acquire vacuum expectation values

$$
\left\langle\phi_{i}\right\rangle=\left(\phi_{0}\right)_{i}
$$

and expanding $\phi_{i}$ around those values, by plugging Eq. (2-26) into Eq. (2-25) gives a mass matrix

$$
m_{a b}^{2}=g^{2}\left(t^{a} \phi_{0}\right)_{i}\left(t^{b} \phi_{0}\right)_{i}
$$

and, with the exception of the gauge bosons related to a generator that coincidentally satisfies $\left(t^{a} \phi_{0}\right)_{i}=0$, all the gauge bosons acquire masses under this mechanism. 


\section{2}

\section{Weak interactions}

After studying the effects of Spontaneous Symmetry Breaking, we are now ready to apply it to the Standard Model in order to understand how the weak interactions work (25), (26), (27). In the Standard Model, the electroweak interactions, which mediates the radioactive decays in nature and is the highenergy unification between the weak force and electromagnetism, is based on the symmetry group

$$
S U(2) \times U(1)_{Y},
$$

where the charge associated with the $U(1)_{Y}$ symmetry is called the Hypercharge $\left(Q_{Y}\right)$ and is related to the electric charge by the following expression

$$
Q=T^{3}+Q_{Y}
$$

where $T^{3}$ is the third representation matrix of $S U(2)$ and $Q$ is the operator that, when acting on a field, gives the electric charge related to this field.

At low energies, $S U(2) \times U(1)_{Y}$ is spontaneously broken down to the electric force when a complex doublet $H$, with hypercharge

$$
Q_{Y}(H)=\frac{1}{2}
$$

called the Higgs doublet, acquires a vacuum expectation value, that is

$$
S U(2) \times U(1)_{Y} \rightarrow U(1)_{e m}
$$

where $U(1)_{e m}$ is the symmetry group of Quantum Electrodynamics (QED) and the related conserved charge is the electric charge $(e)$.

The Lagrangian before SSB can be written as

$$
\mathcal{L}=-\frac{1}{4}\left(W_{\mu}^{a b}\right)^{2}-\frac{1}{4}\left(B_{\mu \nu}\right)^{2}+\left(D_{\mu} H\right)^{\dagger}\left(D_{\mu} H\right)+m^{2} H^{\dagger} H-\lambda\left(H^{\dagger} H\right)^{2},
$$

where $W_{\mu}^{a}$ are the $S U(2)$ gauge bosons, $W_{\mu \nu}^{a b}$ are the related field strengths, $B_{\mu}$ is the hypercharge gauge boson and $B_{\mu \nu}=\partial_{\nu} B_{\mu}-\partial_{\mu} B_{\nu}$ is the hypercharge field strength. The covariant derivative is given by

$$
D_{\mu}=\partial_{\mu}+i g_{1} Q_{Y} B_{\mu}+i g_{2} T^{a} W_{\mu}^{a}
$$

where $g_{1}$ and $g_{2}$ are the couplings for $U(1)_{Y}$ and $S U(2)$, respectively, $Q_{Y}$ is the hypercharge, which is the conserved charge of the symmetry group $U(1)_{Y}$ and $T^{a}$ are the representation matrices of $S U(2)$. Usually the representation of $S U(2)$ chosen under the context of the SM is the fundamental representation, under which

$$
T^{a}=\frac{1}{2} \sigma^{a}
$$


where $\sigma^{a}$ are the Pauli matrices

$$
\sigma^{1}=\left(\begin{array}{cc}
0 & 1 \\
1 & 0
\end{array}\right), \quad \sigma^{2}=\left(\begin{array}{cc}
0 & -i \\
i & 0
\end{array}\right), \quad \sigma^{3}=\left(\begin{array}{cc}
1 & 0 \\
0 & -1
\end{array}\right) .
$$

So one can write, in the fundamental representation of $S U(2)$

$$
D_{\mu}=\partial_{\mu}+i\left(\begin{array}{cc}
g_{1} Q_{Y} B_{\mu}+\frac{g_{2}}{2} W_{\mu}^{3} & \frac{g_{2}}{2}\left(W_{\mu}^{1}-i W_{\mu}^{2}\right) \\
\frac{g_{2}}{2}\left(W_{\mu}^{1}+i W_{\mu}^{2}\right) & g_{1} Q_{Y} B_{\mu}-\frac{g_{2}}{2} W_{\mu}^{3}
\end{array}\right) .
$$

\subsection{1}

\section{The gauge sector}

For the time being, the gauge bosons in the theory are still massless, as can be seen from Eq. (2-31), there are no mass terms for $B_{\mu}$ and $W_{\mu}^{a}$. It has been shown, in the previous section, that a convenient way of giving mass to gauge bosons is via the Higgs mechanism and it is precisely what we aim to do here. So, we wish to minimize the potential in Eq. (2-31)

$$
V(H)=-m^{2}|H|^{2}+\lambda|H|^{4}
$$

which is called the Higgs potential. One can obtain the vacuum expectation value of the Higgs by minimizing Eq. (2-36), which can be taken real without the loss of generality, since the Lagrangian in Eq. (2-31) is invariant under a unitary transformation of the $H$ field. Being so, one can take the VEV for the Higgs doublet as

$$
\langle H\rangle=\frac{1}{\sqrt{2}}\left(\begin{array}{l}
0 \\
v
\end{array}\right)
$$

and, similarly as done previously in Eq. (2-13), perform an expansion around such VEV as

$$
H(x)=\frac{1}{\sqrt{2}}\left(\begin{array}{c}
\phi^{+}(x) \\
v+\rho(x)+i \xi(x)
\end{array}\right)
$$

which can be taken in the unitary gauge as

$$
H(x)=\frac{1}{\sqrt{2}}\left(\begin{array}{c}
0 \\
v+h(x)
\end{array}\right)
$$

where $h$ is the only physical particle, while $\phi, \rho$ and $\xi$ are scalar fields that can be gauged away.

Now, one can investigate what happens with the kinetic terms in Eq. (2-31) after we take the expansion in Eq. (2-39), while also using Eq. (2-35). 
The result is given by

$$
\begin{aligned}
\left|D_{\mu} H\right|^{2} & =\left(\partial_{\mu} H\right)^{2}+\frac{1}{8}\left[\left(\begin{array}{ll}
0 & v+h
\end{array}\right)\left(\begin{array}{cc}
g_{1} B_{\mu}+g_{2} W_{\mu}^{3} & g_{2}\left(W_{\mu}^{1}-i W_{\mu}^{2}\right) \\
g_{2}\left(W_{\mu}^{1}+i W_{\mu}^{2}\right) & g_{1} B_{\mu}-g_{2} W_{\mu}^{3}
\end{array}\right)\right]^{2} \\
& =\left(\partial_{\mu} H\right)^{2}+\frac{(v+h)^{2}}{8}\left[\left(g_{2} W_{\mu}^{1}\right)^{2}+\left(g_{2} W_{\mu}^{2}\right)^{2}+\left(g_{1} B_{\mu}-g_{2} W_{\mu}^{3}\right)^{2}\right] .(2-40)
\end{aligned}
$$

One can note that Eq. (2-40) contains three massive gauge bosons which are coupled to the scalar field $h$ (which is the physical Higgs boson). However, the last term in Eq. (2-40) seems to contain a mass mixing between $B$ and $W$ and, in order to diagonalize the masses one can take the following rotations

$$
\left(\begin{array}{c}
B_{\mu} \\
W_{\mu}^{3}
\end{array}\right)=\left(\begin{array}{cc}
\cos \theta & -\sin \theta \\
\sin \theta & \cos \theta
\end{array}\right)\left(\begin{array}{c}
A_{\mu} \\
Z_{\mu}
\end{array}\right),
$$

where

$$
\tan \theta \equiv \frac{g_{1}}{g_{2}} \Rightarrow g_{1} \cos \theta=g_{2} \sin \theta
$$

$A_{\mu}$ is the photon vector field and $Z_{\mu}$ is one of the the gauge bosons responsible for mediating the weak interactions. By taking the transformations in Eq.(241) and plugging it into Eq. (2-40), one can obtain the masses of the gauge bosons

$$
m_{W}=\frac{g}{2} v, \quad m_{Z}=\frac{g}{2 \cos \theta} v .
$$

And it becomes clear that the masses of the gauge bosons were generated by the VEV of the Higgs doublet.

An important result of the mechanism, which has been successfully confirmed experimentally, is that it predicts that the coupling of the Higgs boson to the other Standard Model fields is proportional to the mass of the field that the Higgs is coupled to. The success of this prediction is illustrated by Fig. 2.3. This behaviour can be seen explicitly by looking at the mass term for the $Z$ boson taken from Eq. (2-40), after the change of basis in Eq. (2-41):

$$
\left|D_{\mu} H\right|^{2}=\ldots+\frac{(v+h)^{2}}{v^{2}} m_{Z}^{2} Z_{\mu} Z^{\mu}=m_{Z}^{2} Z_{\mu} Z^{\mu}+2 \frac{m_{Z}^{2}}{v} h Z_{\mu} Z^{\mu}+\frac{m_{Z}^{2}}{v^{2}} h^{2} Z_{\mu} Z^{\mu},
$$

which clearly shows that the interaction between $h$ and $Z_{\mu}$ is proportional to $m_{Z}^{2}$.

It is worth noting that $W^{1}$ and $W^{2}$ represent the same particle, since one can define

$$
\sigma^{ \pm} \equiv \frac{1}{\sqrt{2}}\left(\sigma^{1} \pm i \sigma^{2}\right), \quad W_{\mu}^{ \pm} \equiv \frac{1}{\sqrt{2}}\left(W_{\mu}^{1} \mp i W_{\mu}^{2}\right),
$$

this way, the following relation arises

$$
\sigma^{1} W_{\mu}^{1}+\sigma^{2} W_{\mu}^{2}=\sigma^{+} W_{\mu}^{+}+\sigma^{-} W_{\mu}^{-} .
$$




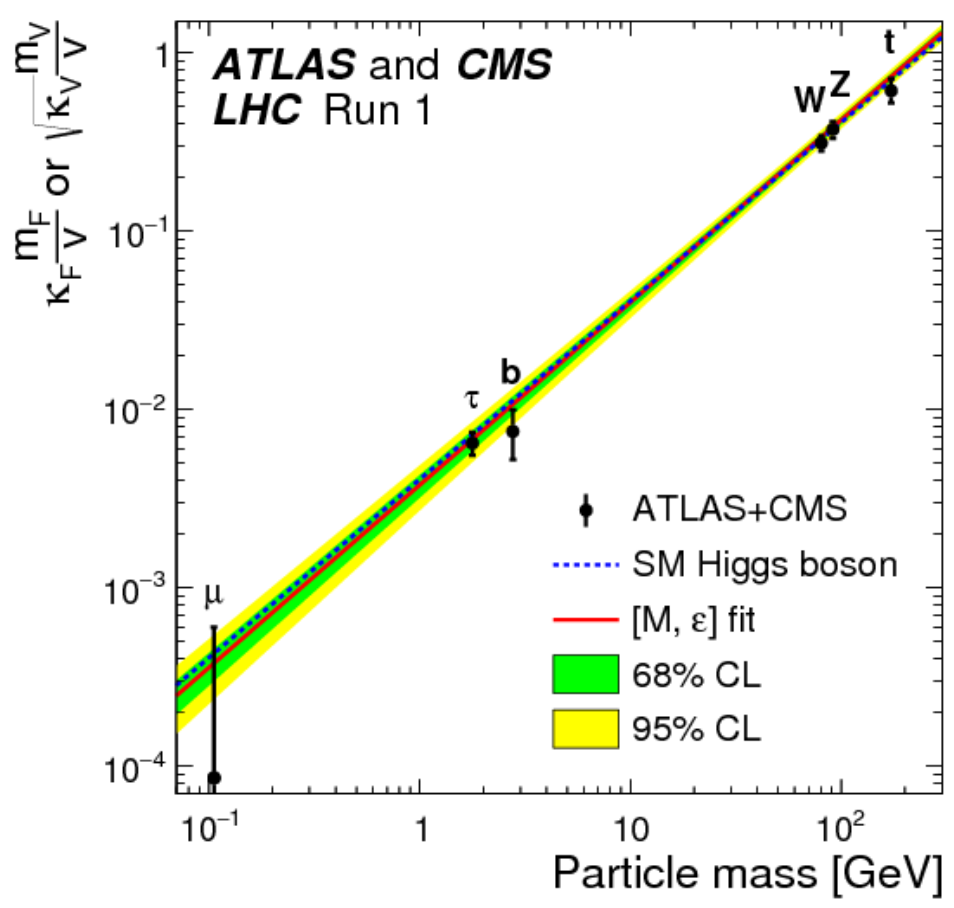

Figure 2.3: Best fit values of the Higgs boson coupling to different particles as a function of particle mass for the combination of ATLAS and CMS data (28)

One can obtain a relation between the electric charge $e$ and the angle $\theta$ by taking the transformation in Eq. (2-41) and plug it into the covariant derivative in Eq. (2-32)

$$
\begin{aligned}
D_{\mu}=\partial_{\mu}+\left(i g_{1} Q_{Y} \cos \theta+i g_{2} T^{3} \sin \theta\right) A_{\mu}+ & \left(i g_{2} T^{3} \cos \theta-i g_{1} Q_{Y} \sin \theta\right) Z_{\mu} \\
& +i g_{2} T^{1} W_{\mu}^{1}+i g_{2} T^{2} W_{\mu}^{2}
\end{aligned}
$$

One can see in Eq. (2-47) that the $A_{\mu}$ term can be rewriten as

$$
g_{1} \cos \theta\left(Q_{Y}+T^{3}\right) A_{\mu}=g_{1} \cos \theta Q A_{\mu},
$$

where we have used Eq. (2-29). Now, since $Q$ is the electric charge operator, one should expect a term like $e Q A_{\mu}$ in the the covariant derivative, this way, one can safely conclude that

$$
e=g_{1} \cos \theta=g_{2} \sin \theta
$$

\subsection{2}

\section{The fermion sector}

The theory of weak interactions is unique in the sense that it happens to be a chiral symmetry, which means that only left-handed fermions couple 
to the $S U(2)$ gauge bosons. Such property was only observed experimentally and incorporated in the SM thereafter. This chiral characteristic of the theory is the main source of parity violation in nature.

The left-handed fermions are paired up in doublets in the fundamental representation of $S U(2)$, while the right-handed ones are singlets under such symmetry. Their representation can be writen as:

$$
q_{L}^{i}=\left(\begin{array}{c}
u_{L} \\
d_{L}
\end{array}\right),\left(\begin{array}{c}
c_{L} \\
s_{L}
\end{array}\right),\left(\begin{array}{c}
t_{L} \\
b_{L}
\end{array}\right), \quad l_{L}^{i}=\left(\begin{array}{c}
\nu_{e L} \\
e_{L}
\end{array}\right),\left(\begin{array}{c}
\nu_{\mu_{L}} \\
\mu_{L}
\end{array}\right),\left(\begin{array}{c}
\nu_{\tau L} \\
\tau_{L}
\end{array}\right),
$$

where $i=\{1,2,3\}$ is a flavour index. For the right-handed singlets:

$$
\begin{gathered}
u_{R}^{i}=\left\{u_{R}, c_{R}, t_{R}\right\}, \quad d_{R}^{i}=\left\{d_{R}, s_{R}, b_{R}\right\}, \\
e_{R}^{i}=\left\{e_{R}, \mu_{R}, \tau_{R}\right\}, \quad \nu_{R}^{i}=\left\{\nu_{e R}, \nu_{\mu_{R}}, \nu_{\tau R}\right\} .
\end{gathered}
$$

Here, even though it is not really part of the SM as it has not yet been detected, we have introduced a right-handed neutrino field, as it will be needed later to construct the see-saw mechanism in order to give small masses to the neutrinos.

Now, in possession of the fields from Eq. (2-51) and Eq. (2-52), one can write the following Lagrangian for the fermion interactions with the gauge bosons:

$$
\begin{gathered}
\mathcal{L}=i \bar{l}_{L}^{i}\left(\not \partial+i g_{1} Q_{Y}^{l} \not B+i g_{2} W^{a} T^{a}\right) l_{L}^{i}+i \bar{q}_{L}^{i}\left(\not \partial+i g_{1} Q_{Y}^{q} \not B+i g_{2} W^{a} T^{a}\right) q_{L}^{i} \\
+i \bar{u}_{R}^{i}\left(\not \partial+i g_{1} Q_{Y}^{u} \not B\right) u_{R}^{i}+i \bar{d}_{R}^{i}\left(\not \partial+i g_{1} Q_{Y}^{d} \not B\right) d_{R}^{i} \\
+i \bar{e}_{R}^{i}\left(\not \partial+i g_{1} Q_{Y}^{e} \not B\right) e_{R}^{i}+i \bar{\nu}_{R}^{i}\left(\not \partial+i g_{1} Q_{Y}^{\nu} \not B\right) \nu_{R}^{i},
\end{gathered}
$$

where $Q_{Y}$ is the hypercharge and $T^{a}$ are the $S U(2)$ generators. The hypercharge adopted for the fermions fields and the corresponding multiplicity under $S U(2)$ are displayed in Table 2.1. This way, when acting with the operator

$$
Q=T^{3}+Q_{Y}
$$

on the fermion fields, one can consistently reproduce the correct electric charges for such fermions.

Now, the only thing missing in the Lagrangian from Eq. (2-53) is the fermion mass term. One might be tempted to simply write mass terms such as $m_{e} \bar{e}_{L} e_{R}$ however, as one can see from the charge assignment in Table 2.1, these terms are not invariant under $S U(2)$. The solution to this problem is to write Yukawa interactions mediated by the Higgs doublet $(H)$, such as:

$$
\mathcal{L}_{Y u k}=\bar{\psi}_{L}^{i} Y_{i j} H \chi_{R}^{j}
$$


Table 2.1: Hypercharge and multiplicity of fields under SU(2). The notation here can be clarified by the example $2_{-\frac{1}{2}}$, which means that the field transforms as a doublet under $S U(2)$ and has hypercharge $-\frac{1}{2}$.

\begin{tabular}{cc}
\hline Field & $S U(2) \times U(1)_{Y}$ \\
\hline$q_{L}$ & $2_{\frac{1}{6}}$ \\
$l_{L}$ & $2_{-\frac{1}{2}}$ \\
\hline$u_{R}$ & $1_{\frac{2}{3}}$ \\
$d_{R}$ & $1_{-\frac{1}{3}}$ \\
$e_{R}$ & $1_{-1}$ \\
$\nu_{R}$ & $1_{0}$ \\
\hline$H$ & $2_{\frac{1}{2}}$ \\
\hline
\end{tabular}

where $\psi_{L}$ is a left-handed doublet, $\chi$ is right-handed singlet and $Y$ is the Yukawa interaction between them. For example, if one takes $\psi_{L}=q_{L}$ and $\chi_{R}=d_{R}$ in Eq. (2-55), the hypercharge of the result would be:

$$
Q_{Y}=-\frac{1}{6}+\frac{1}{2}-\frac{1}{3}=0
$$

however, the same procedure does not yield a null hypercharge if one takes $\left(\psi_{L}=q_{L}, \chi_{R}=u_{R}\right)$ or $\left(\psi_{L}=l_{L}, \chi_{R}=\nu_{R}\right)$ in Eq. (2-55), in that case, in order to get proper invariant mass terms, one needs to couple the Yukawa interactions with a transformation of the Higgs doublet:

$$
\tilde{H} \equiv i \sigma_{2} H^{*}, \quad\langle\tilde{H}\rangle=\left(\begin{array}{c}
\frac{v}{\sqrt{2}} \\
0
\end{array}\right)
$$

which also transforms as a doublet of $S U(2)$ but with $Q_{Y}=-\frac{1}{2}$.

\subsubsection{1}

\section{The quark sector}

We are now ready to write the full Lagrangian for the quark sector:

$$
\mathcal{L}_{\text {quarks }}=i \bar{q}_{L} \not D q_{L}+i \bar{d}_{R} \not D d_{R}+i \bar{u}_{R} \not D u_{R}+\bar{q}_{L} Y_{d} H d_{R}+\bar{q}_{L} Y_{u} \tilde{H} u_{R}+\text { h.c. . }
$$

Here, if it were not for the gauge interactions, one could diagonalize the Yukawa matrices and the masses obtained would be the only physical parameters. In any case, one can find physical masses in addition to other parameters, by going to the mass base, in which the Yukawas are diagonal. To accomplish 
such task, one can use singular value decomposition to write:

$$
Y_{d}=U_{d} \tilde{Y}_{d} K_{d}^{\dagger}, \quad Y_{u}=U_{u} \tilde{Y}_{u} K_{u}^{\dagger}
$$

where $U$ and $K$ are unitary matrices and

$$
\tilde{Y}_{d}=\left(\begin{array}{ccc}
y_{d} & & \\
& y_{s} & \\
& & y_{b}
\end{array}\right), \quad \tilde{Y}_{u}=\left(\begin{array}{ccc}
y_{u} & & \\
& y_{c} & \\
& & y_{t}
\end{array}\right),
$$

where $y$ represents the Yukawa eigenvalues. Plugging Eq. (2-58) into Eq. (257 ), while also taking the symmetry breaking of the Higgs doublet, gives

$$
\begin{aligned}
\mathcal{L}_{\text {quarks }}=i \bar{q}_{L} \not D q_{L}+i \bar{d}_{R} \not D d_{R}+i \bar{u}_{R} \not D u_{R} & +\frac{v^{2}}{2} \bar{d}_{L}\left(U_{d} \tilde{Y}_{d} K_{d}^{\dagger}\right) d_{R} \\
& +\frac{v^{2}}{2} \bar{u}_{L}\left(U_{u} \tilde{Y}_{u} K_{u}^{\dagger}\right) u_{R}+\text { h.c. }
\end{aligned}
$$

Now one can perform the following transformations:

$$
\begin{gathered}
d_{L} \rightarrow U_{d} d_{L}, \quad u_{L} \rightarrow U_{u} u_{L}, \\
d_{R} \rightarrow K_{d} d_{R}, \quad u_{R} \rightarrow K_{u} u_{R},
\end{gathered}
$$

in order to remove the unitary matrices from the Yukawa terms and obtain quark masses as

$$
m_{d}^{j}=\frac{v}{\sqrt{2}} y_{d}^{j}, \quad m_{u}^{j}=\frac{v}{\sqrt{2}} y_{u}^{j},
$$

where $j$ is a flavour index. However these transformations will cause the unitary matrices $U$ to move to the gauge interaction terms, which will have the effect of mixing the flavours under such interactions. Expanding the kinetic terms in Eq. (2-57), using

$$
\not D=\not D+i \frac{g_{2}}{2} W^{+} \sigma^{+}+i \frac{g_{2}}{2} W^{-} \sigma^{+}+i \frac{g_{2}}{2} W^{3} \sigma^{3}+i g_{1} Q_{Y} \not B
$$

gives:

$$
\begin{aligned}
\mathcal{L}_{k i n}=\left(\begin{array}{ll}
\bar{u}_{L} \quad \bar{d}_{L}
\end{array}\right)^{i}[i \not \partial & \left.+\left(\begin{array}{cc}
\frac{g_{1}}{6} \not B+\frac{g_{2}}{2} \not^{3} & \frac{g_{2}}{\sqrt{2}} W^{+} \\
\frac{g_{2}}{\sqrt{2}} \mathscr{W}^{-} & \frac{g_{1}}{6} \not B-\frac{g_{2}}{2} W^{3}
\end{array}\right)\right]\left(\begin{array}{c}
u_{L} \\
d_{L}
\end{array}\right)^{i} \\
& +\bar{u}_{R}^{i}\left(i \not \partial+g_{1} \frac{2}{3} \not B\right) u_{R}^{i}+\bar{d}_{R}^{i}\left(i \not \partial-g_{1} \frac{1}{3} \not B\right) d_{R}^{i}
\end{aligned}
$$

Applying the flavour rotations from Eq. (2-61) and Eq. (2-62) in Eq. (2-65) would not affect the right-handed kinetic terms, since $\not B$ is flavour diagonal, on the other hand, the left-handed gauge interactions will be modified, since $W^{ \pm}$ are not diagonal in flavour space, and so the resulting Lagrangian will contain a quark mixing term like: 


$$
\mathcal{L}_{\text {mixing }}=\frac{g}{\sqrt{2}} W^{+} \bar{u}_{L}^{i} V_{i j} d_{L}^{j}+\text { h.c. }
$$

where

$$
V \equiv V_{C K M}=U_{u}^{\dagger} U_{d}=\left(\begin{array}{ccc}
V_{11} & V_{12} & V_{13} \\
V_{21} & V_{22} & V_{23} \\
V_{31} & V_{32} & V_{33}
\end{array}\right)=\left(\begin{array}{ccc}
V_{u d} & V_{u s} & V_{u b} \\
V_{c d} & V_{c s} & V_{c b} \\
V_{t d} & V_{t s} & V_{t b}
\end{array}\right)
$$

This effect causes flavour changing processes mediated by a $W$ boson, and the strength of such processes is dictated by the entries in the mixing matrix $V_{C K M}$, called CKM matrix, after Cabibbo, Kobayashi and Maskawa (29), (30).

It is worth noting, that even after the flavour rotations performed via the transformations in Eq. (2-61) and Eq. (2-62), the resulting mass Lagrangian still maintains a residual $U(1)^{6}$ symmetry, as individual rotations for each generation, such as:

$$
\begin{aligned}
& d_{L}^{j} \rightarrow e^{i \alpha_{j}} d_{L}^{j}, \quad d_{R}^{j} \rightarrow e^{i \alpha_{j}} d_{R}^{j}, \\
& u_{L}^{j} \rightarrow e^{i \beta_{j}} d_{L}^{j}, \quad u_{R}^{j} \rightarrow e^{i \beta_{j}} u_{R}^{j} .
\end{aligned}
$$

Since the CKM Matrix is a $3 \times 3$ unitary matrix, it has $3^{2}=9$ degrees of freedom, three of those being angles and six phases. Using the aforementioned $U(1)^{6}$ symmetry, one can remove some phases, while transforming the mixing matrix in the process. However, if one takes all the rotations to be the same, the matrix will remain unchanged, since an overall phase is irrelevant for all purposes. Therefore, only up to five phases can be removed this way. After this operation, four real degrees of freedom will remain, three angles $\left(\theta_{12}, \theta_{13}\right.$ and $\left.\theta_{23}\right)$ and one phase $\left(\delta_{13}\right)$. The standard way to write the CKM in terms of these parameters is the following:

$$
\begin{gathered}
V_{C K M}=\left(\begin{array}{ccc}
1 & & \\
& c_{23} & s_{23} \\
-s_{23} & c_{23}
\end{array}\right)\left(\begin{array}{ccc}
c_{13} & & s_{13} e^{-i \delta_{13}} \\
& 1 & \\
-s_{13} e^{i \delta_{13}} & c_{13}
\end{array}\right)\left(\begin{array}{ccc}
c_{12} & s_{12} & \\
-s_{12} & c_{12} & \\
& & 1
\end{array}\right) \\
=\left(\begin{array}{ccc}
c_{12} c_{13} & s_{12} c_{13} & s_{13} e^{-i \delta_{13}} \\
-s_{12} c_{23}-c_{12} s_{23} s_{13} e^{i \delta_{13}} & c_{12} c_{23}-s_{12} s_{23} s_{13} e^{i \delta_{13}} & s_{23} c_{13} \\
s_{12} s_{23}-c_{12} c_{23} s_{13} e^{i \delta_{13}} & -c_{12} s_{23}-s_{12} c_{23} s_{13} e^{i \delta_{13}} & c_{23} c_{13}
\end{array}\right), \quad(2-70)
\end{gathered}
$$

where $s_{i j} \equiv \sin \theta_{i j}$ and $c_{i j} \equiv \cos \theta_{i j}$. These mixing angles have been measured to be noticeably small and the mixing in the quark sector can be regarded as weak. This way, flavour changing process are bound to occur seldomly. The experimentally measured values for the mixing angles in the quark sector are shown in Table $2.3(7)$. 
It is also worth noting that, since one can show that if the CKM matrix were real the Lagrangian in Eq. (2-57) would be $C P$ invariant, the phase $\delta_{13}$ is the only source of $C P$ violation in the quark sector. A usual way of quantifying the $C P$ violation in the quark sector is via a parameter $J$ known as the Jarlskog invariant and it can be expressed in terms of the standard parametrization as

$$
J=s_{12} s_{23} s_{31} c_{12} c_{23} c_{31}^{2} \sin \delta_{13} .
$$

This parameter was measured to be small $\left(J \sim 10^{-5}\right)$ and it is the source of weak $C P$ violation in the Standard Model. The experimental value for $J$ is shown in Table $2.3(7)$.

The measured quark masses are presented in Table 2.2 (7), which constitute undeniable evidence for the hierarchical spectra across the generations as well as between the up and down sectors and is the main motivation for this work.

Table 2.2: Experimentally measured values for the quark masses (7). The hierarchy across families and between the up and down sector is clear. The experimental errors involved are ommited due to the fact that their contribution is neglectable for the development of the present work.

\begin{tabular}{cc}
\hline Quark & Mass $(\mathrm{MeV})$ \\
\hline$u$ & 2.2 \\
$c$ & 1275 \\
$t$ & 173000 \\
\hline$d$ & 4.7 \\
$s$ & 95 \\
$b$ & 4180 \\
\hline
\end{tabular}

Table 2.3: Experimentally measured values for the mixing angles and the Jarlskog invariant in the quark sector (7). The mixing angles are clearly very small, and the CKM matrix can be taken as almost diagonal. The experimental errors involved are ommited due to the fact that their contribution is neglectable for the development of the present work.

\begin{tabular}{cc}
\hline Parameter & Value \\
\hline$\theta_{12}$ & $12.98^{\circ}$ \\
$\theta_{23}$ & $2.42^{\circ}$ \\
$\theta_{13}$ & $0.20^{\circ}$ \\
\hline$J$ & $(3.18 \pm 0.15) \times 10^{-5}$ \\
\hline
\end{tabular}




\subsubsection{2}

\section{The lepton sector and the see-saw mechanism}

The lepton sector is built in a similar configuration as the quark sector, the only difference behaviour comes from the existence of light particles, called neutrinos, which have the particular property of oscillating between different flavours $\left(\nu_{e}, \nu_{\mu}\right.$ and $\left.\nu_{\tau}\right)$ which are written as linear combinations of three possible mass eigenstates $\nu_{1}, \nu_{2}$ and $\nu_{3}$.

One must recall that there is no right-handed neutrino field $\left(\nu_{R}\right.$, often referred as sterile neutrinos) in the SM per se, as its existence has not yet been confirmed experimentally, however, more generally, since there is no detected symmetry that forbids its existence and introducing sterile neutrinos is a very useful way of generating small neutrinos masses via the see-saw mechanism (31), (32), (33), (34) one can assume the presence of right-handed neutrinos fields in a theoretical model.

Since it is not forbidden by electroweak symmetry, one can include Majorana mass terms for sterile neutrinos (35), (36) (this can only be done because, unlike the other SM fields, $\nu_{R}$ is a chargeless singlet in $\left.S U(2) \times U(1)_{Y}\right)$ in addition to the Dirac mass terms in the Lagrangian, such as

$\mathcal{L}_{\text {leptons }}=i \bar{l}_{L} \not D l_{L}+i \bar{e}_{R} \not D e_{R}+i \overline{\nu_{R}} \not D \nu_{R}+\bar{l}_{L} Y_{e} H e_{R}+\bar{l}_{L} Y_{\nu} \tilde{H} \nu_{R}+\bar{\nu}_{R} M_{\nu_{R}} \nu_{R}^{c}+$ h.c.,

where $M_{\nu_{R}}$ is a Majorana mass matrix for right-handed neutrinos and $\nu_{R}^{c}$ is the charge conjugate Weyl spinor

$$
\nu_{R}^{c}=i \sigma_{2} \nu_{R}^{T} .
$$

One can use Weyl spinors to make a uniform notation and unify the Dirac mass term with the Majorana mass term in a single matrix (35) as

$$
M_{\nu}=\left(\begin{array}{ll}
\bar{\nu}_{L} & \bar{\nu}_{R}^{c}
\end{array}\right)\left(\begin{array}{cc}
0 & \frac{v}{\sqrt{2}} Y_{\nu} \\
\frac{v}{\sqrt{2}} Y_{\nu}^{T} & M_{\nu_{R}}
\end{array}\right)\left(\begin{array}{c}
\nu_{L}^{c} \\
\nu_{R}
\end{array}\right)
$$

where it is taken as an assumption that there is no left-handed neutrino Majorana mass term, however, it can be generated effectively by diagonalizing the mass matrix in Eq. (2-74) and yielding

$$
M_{\nu_{L}}=\frac{v^{2}}{2} Y_{\nu} M_{\nu_{R}}^{-1} Y_{\nu}^{T}
$$

where the approximation

$$
M_{\nu_{R}} \gg \frac{v^{2}}{2} Y_{\nu}
$$

was used, since the right-handed neutrinos are electroweak singlets and the Majorana masses of the right-handed neutrinos $M_{\nu_{R}}$ may be orders of magnitude 
larger than the electroweak scale. This way, the Majorana mass in Eq. (2-75) will contain naturally suppressed mass eingenvalues, while $M_{\nu_{R}}$ will contain heavy mass eigenvalues (a bit below the GUT scale, usually taken as $\sim 10^{16}$ $\mathrm{GeV}$ ) and one can see that as $M_{\nu_{R}}$ goes up $M_{\nu_{L}}$ goes down. This effect can explain the lightness of the neutrino masses $(<0.1 \mathrm{eV})$ and is given the name of see-saw mechanism.

Now, if one goes through the same basis change procedure and move to the mass base, as we did in the quark sector, once again a mixing matrix coupled to the $W$ boson would appear via the transformations in the gauge sector. This matrix is called PMNS matrix, after Pontecorvo, Maki, Nakagawa and Sakata (37), (38), and it is the cause of neutrino oscillations, as it relates the flavoured neutrinos as linear combinations of the eigenstates $\nu_{1}, \nu_{2}$ and $\nu_{3}$, that is

The PMNS matrix is given by

$$
\left(\begin{array}{c}
\nu_{e} \\
\nu_{\mu} \\
\nu_{\tau}
\end{array}\right)=V_{P M N S}\left(\begin{array}{l}
\nu_{1} \\
\nu_{2} \\
\nu_{3}
\end{array}\right) .
$$

$$
V_{P M N S}=U_{\nu}^{\dagger} U_{e}
$$

where $U_{\nu}$ and $U_{e}$ are unitary matrices that diagonalize $M_{\nu_{L}}$ and $Y_{e}$, respectively, that is

$$
\begin{gathered}
\tilde{M}_{\nu_{L}}=U_{\nu} M_{\nu_{L}} U_{\nu}^{T}, \\
\tilde{Y}_{e}=U_{e} Y_{e} K_{e}^{\dagger},
\end{gathered}
$$

where $U$ and $K$ are unitary matrices and $\tilde{M}_{\nu_{L}}$ and $\tilde{Y}_{e}$ are diagonal matrices. The standard parametrization of the PMNS matrix is given by

$$
\begin{array}{r}
c_{12} c_{13} \\
V_{P M N S}=\left(\begin{array}{ccc}
s_{12} c_{13} & s_{13} e^{-i \delta_{13}} \\
-s_{12} c_{23}-c_{12} s_{23} s_{13} e^{i \delta_{13}} & c_{12} c_{23}-s_{12} s_{23} s_{13} e^{i \delta_{13}} & s_{23} c_{13} \\
s_{12} s_{23}-c_{12} c_{23} s_{13} e^{i \delta_{13}} & -c_{12} s_{23}-s_{12} c_{23} s_{13} e^{i \delta_{13}} & c_{23} c_{13}
\end{array}\right) \\
\times\left(\begin{array}{cc}
1 & \\
& e^{i \frac{\alpha_{12}}{2}} \\
& \\
& e^{i \frac{\alpha_{31}}{2}}
\end{array}\right), \quad(2-80)
\end{array}
$$

where $s_{i j} \equiv \sin \theta_{i j}$ and $c_{i j} \equiv \cos \theta_{i j}$. Additional phases, $\alpha_{12}$ and $\alpha_{31}$, are possible in the lepton sector if Majorana masses are present (for Dirac neutrinos these additional phases can be excluded).

Unlike the mixing angles from the quark sector, the mixing angles from the PMNS matrix are not generally small, and the matrix can not be taken as almost diagonal. The experimental values for the neutrino mixing angles as well as a quantity analogous to the Jarlskog invariant (7) defined as 


$$
J_{C P} \equiv \operatorname{Im}\left(V_{\mu_{3}} V_{e_{3}}^{*} V_{e_{2}} V_{\mu_{2}}^{*}\right)=\frac{1}{8} \cos \theta_{13} \sin 2 \theta_{12} \sin 2 \theta_{23} \sin 2 \theta_{13} \sin \delta,
$$

are shown is Table 2.4, while the measured values for the lepton masses are shown in Table 2.5 .

Table 2.4: Experimentally measured values for the mixing angles and the $J_{C P}$ invariant in the lepton sector (7). The mixing angles are not small, and the CKM matrix can not be taken as almost diagonal. Here, normal ordering was considered and $\theta_{23}$ was taken in the first octant. The experimental errors involved are ommited due to the fact that their contribution is neglectable for the development of the present work.

\begin{tabular}{cc}
\hline Parameter & Value \\
\hline$\theta_{12}$ & $33.65^{\circ}$ \\
$\theta_{23}$ & $40.22^{\circ}$ \\
$\theta_{13}$ & $8.37^{\circ}$ \\
\hline$J_{C P}$ & -0.030 \\
\hline
\end{tabular}

Table 2.5: Experimentally measured values for the lepton masses (7). The hierarchy across families and between the up sector and the charged leptons is clear (see Table 2.2 for comparison). Note that, at the time of this work, neutrino masses have not yet been measured, only the difference between squared masses have been experimentally verified, that is $\Delta m_{i j}^{2} \equiv\left|m_{i}^{2}-m_{j}^{2}\right|$. The experimental errors involved are ommited due to the fact that their contribution is neglectable for the development of the present work.

\begin{tabular}{cc}
\hline Lepton & Mass \\
\hline$e$ & $0.51 \mathrm{MeV}$ \\
$\mu$ & $105.66 \mathrm{MeV}$ \\
$\tau$ & $1776.86 \mathrm{MeV}$ \\
\hline$\Delta m_{12}^{2}$ & $7.53 \times 10^{-5} \mathrm{eV}^{2}$ \\
$\Delta m_{23}^{2}$ & $2.51 \times 10^{-3} \mathrm{eV}^{2}$ \\
\hline
\end{tabular}

\section{3}

\section{Simulations for the Standard Model free parameters in the fermion sector}

One can count the number of free parameters in the fermion sector and see that, in the quark sector, there are 6 quark masses plus 3 mixing angles plus 1 mixing phase, while in the lepton sector, there are 3 charged lepton masses (as we discussed before, in the SM there are no mass terms for the neutrinos) plus 3 mixing angles plus 1 mixing phase. These brings up to a total of 17 free parameters in the Standard Model and, as the values exposed in Table 
2.2, Table 2.3, Table 2.5, and Table 2.4 can confirm, these parameters seems to have acquire very specific values that obey several hierarchical structures, even though there is no mathematical structure of the symmetry groups that can predict such behaviour.

To give an understanding to how these values feel very unnatural in the Standard Model, one can run a simulation with order-one Yukawas, and see how the experimentally measured values fall under the simulated distributions (similar to what have been done in (39)). With the intention of trying to reproduce a natural configuration, the entries in the Yukawa matrices were taken as random complex numbers with a uniform distribution contained within the square of side 1 in the complex plane as seen in Fig. 2.4, this proceeding was repeated for all the subsequent simulations performed. In order to provide a fair comparison with the Clockwork Model, as it will be shown later, here we introduced the parameters $c_{u}, c_{d}$ and $c_{e}$, which are factors that multiply the up, down and charged lepton Yukawas, respectively. These parameters were determined in the simulation via a best fit with the experimental data, according to the $\chi^{2}$ test. According to this test, one finds the best fitted parameters by minimizing the expression

$$
\chi^{2}\left(c_{i}\right)=\sum_{i, j} \frac{\left(x_{i}^{e x p}-c_{i}\left\langle x_{i}\right\rangle\right)}{\sigma_{x_{i}}} C_{i j} \frac{\left(x_{j}^{e x p}-c_{j}\left\langle x_{j}\right\rangle\right)}{\sigma_{x_{j}}},
$$

where $x_{i}^{e x p}$ is the experimental value for the physical quantity at hand, $x_{i}$ is the corresponding simulated distribution, $\sigma_{x_{i}}$ is the standard deviation of such distribution, $C_{i j}$ is the correlation matrix between $x_{i}$ and $x_{j}$ and $c_{i}$ is the parameter introduced to account for the hierarchy between different sectors. Then the parameters $c_{i}$ are determined by solving the system of equations

$$
\frac{\partial}{\partial c_{i}} \chi^{2}\left(c_{i}\right)=0
$$

which guarantees the minimum value for $\chi^{2}$, by taking

$$
\chi_{\min }^{2}=\chi^{2}\left(c_{i}^{o p t}\right),
$$

where $c_{i}^{\text {opt }}$ are the solutions for Eq. (2-83). It is important to note that the experimental errors related to the measurement of the parameters of the SM were not taken into account in any of the expressions for the $\chi^{2}$ test considered in the scope of this work, as their contribution for the calculation of the best fit for the simulations performed is neglectable. The values found for the free parameters in the simulation as displayed in Table 2.6.

The results can be seen in Fig. 2.5, Fig. 2.6, Fig. 2.7 and Fig. 2.8 and almost all of the distributions seem to have fallen way off the experimental 


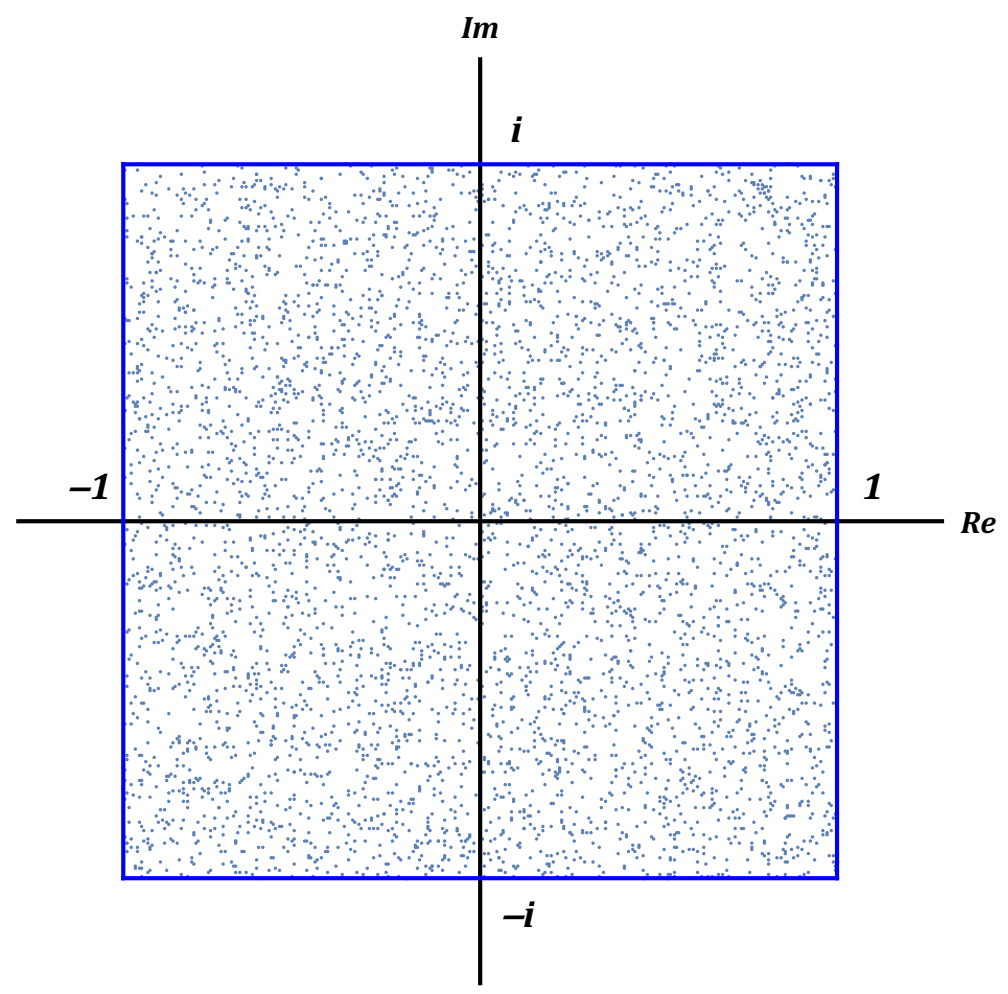

Figure 2.4: Uniform distribution of the entries in the Yukawa matrices. The distribution is contained within a region in the shape of a square with side 1. This distribution garantees that the simulation will reproduce a natural configuration containing only $\mathcal{O}(1)$ Yukawas.

Table 2.6: Best fit free parameters for the SM distribution, in order to attempt to account for the hierarchical structure of fermion masses. The values computed for $\chi_{\min }^{2}$ are way too high, which can be interpreted as a extremely low likelihood of obtaining the experimental values from the simulated distributions.

\begin{tabular}{cc}
\hline Quantity & Value \\
\hline$c_{u}$ & 0.03 \\
$c_{d}$ & $1.5 \times 10^{-3}$ \\
$c_{e}$ & $1.5 \times 10^{-3}$ \\
\hline$\left(\chi_{\text {min }}^{2}\right)^{\text {quark }}$ & 1442 \\
$\left(\chi_{\text {min }}^{2}\right)^{\text {lepton }}$ & 359 \\
\hline
\end{tabular}

data, which confirms that the measured data are highly unnatural under the Standard Model. If one accepts the applied distributions as the most natural ones, it is here that lies the fermion hierarchy problem, which is visually explicit in the plots in this section, that this work attempt to overcome by the later introduction of the Clockwork model. 


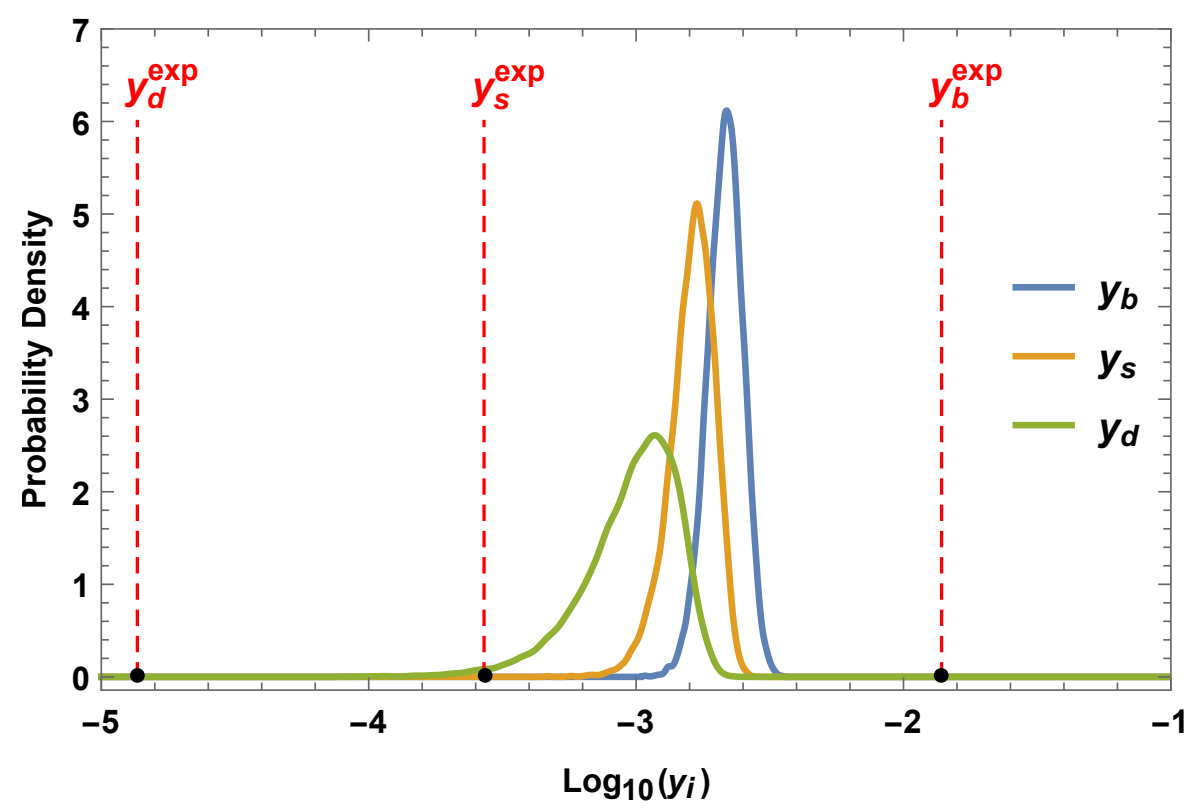

(a) Down sector masses

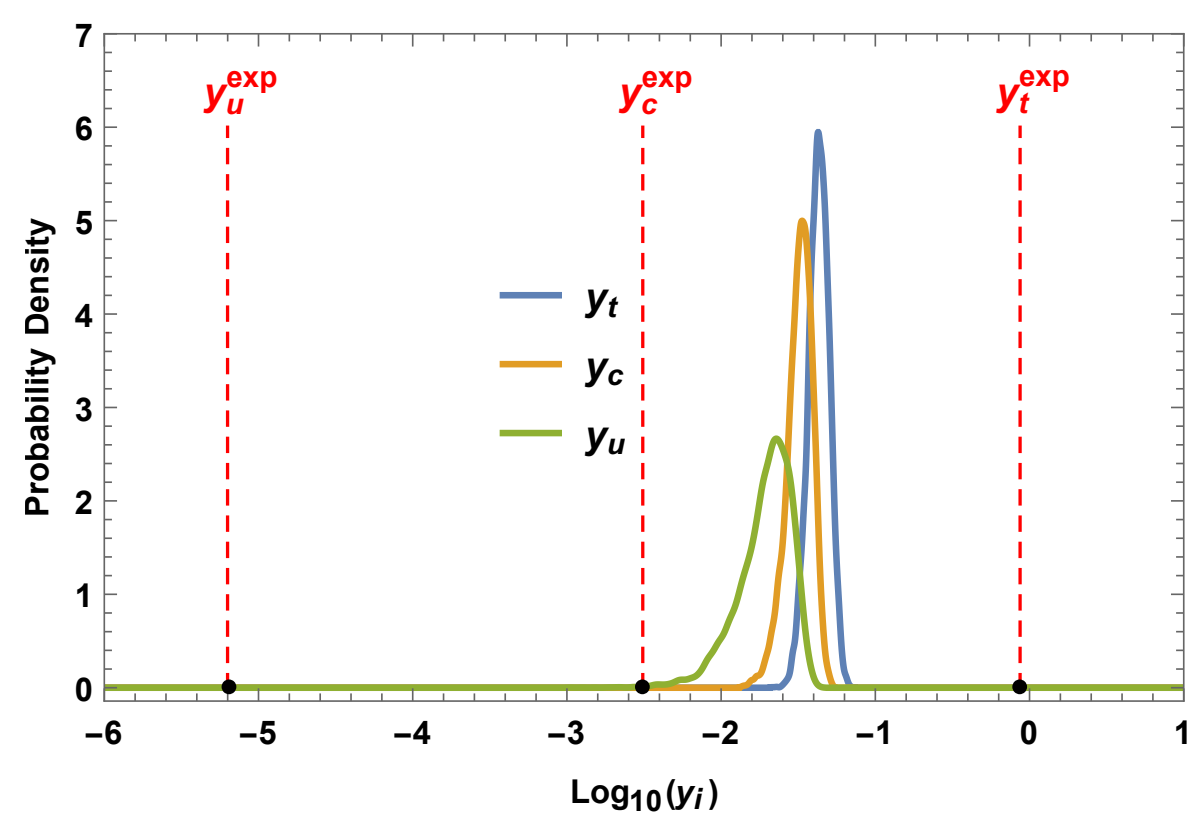

(b) Up sector masses

Figure 2.5: Distributions for the quark masses under the SM, while considering $\mathcal{O}(1)$ Yukawas. Here it becomes clear how such measured experimental values for the down sector masses are very unlikely to randomly be generated under the SM configuration. 


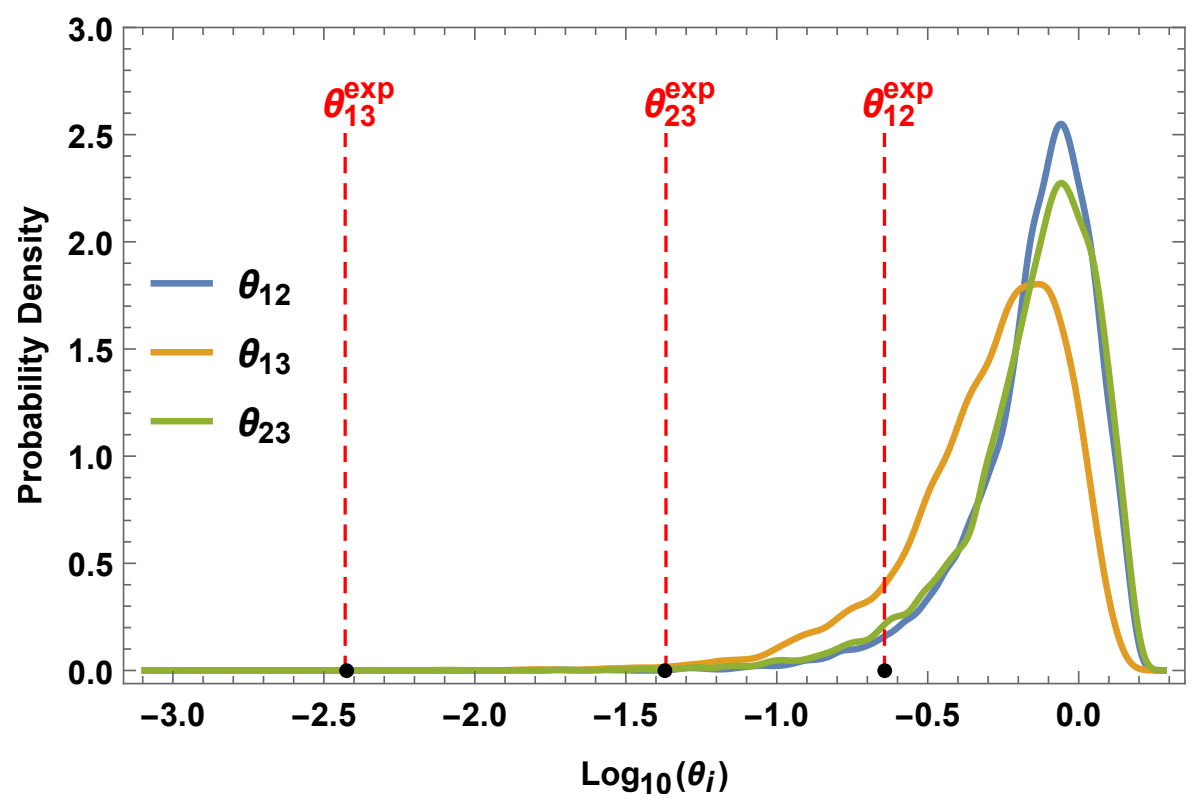

(a) CKM mixing angles

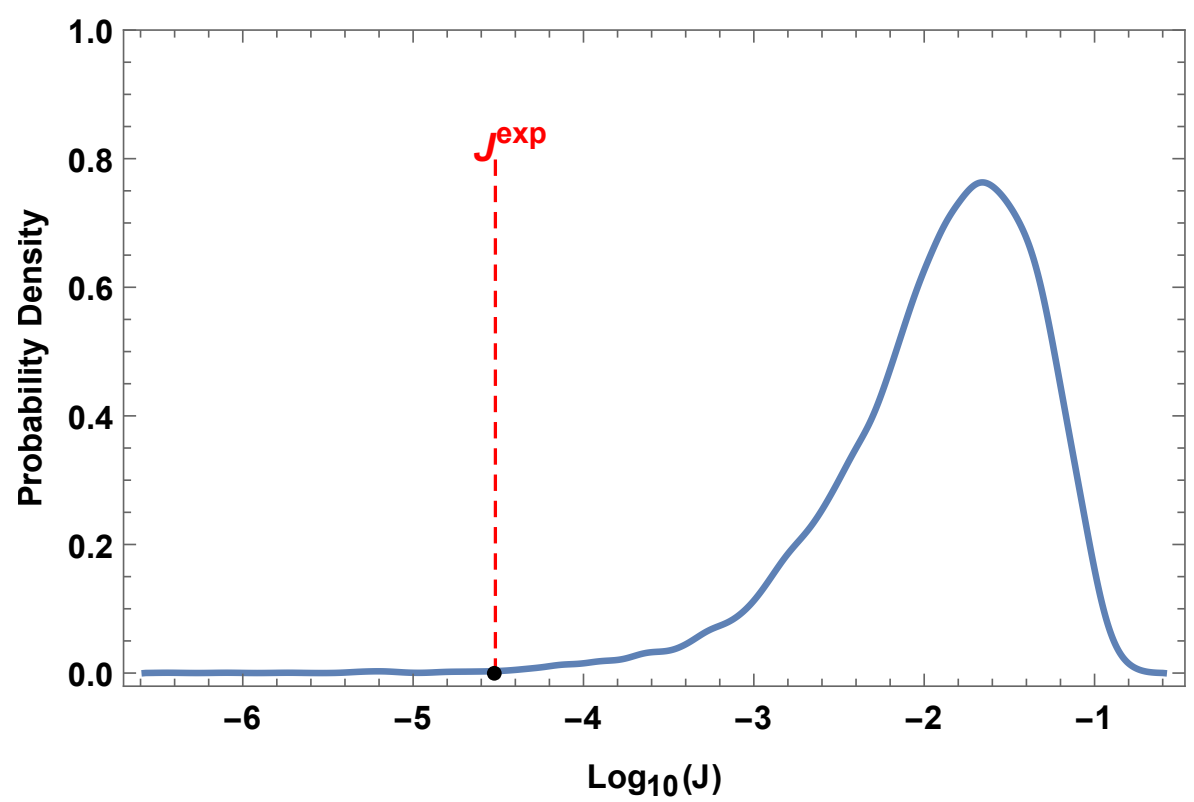

(b) Jarlskog invariant

Figure 2.6: Distributions for the quark mixing parameters under the SM, while considering $\mathcal{O}(1)$ Yukawas. Here it is clear that the small nature of the CKM mixing angles are very hard to come by according to the distribution for the $\mathrm{SM}$ 


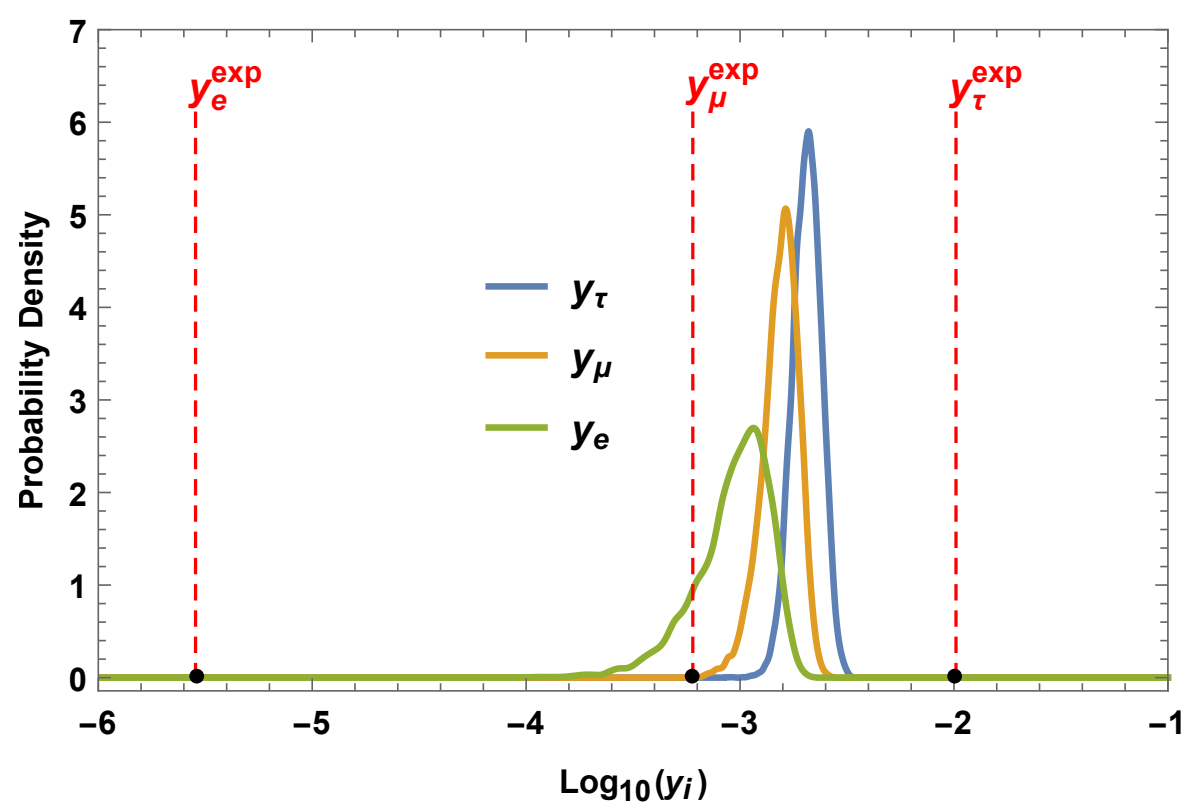

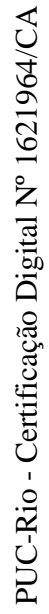

(a) Charged leptons sector masses

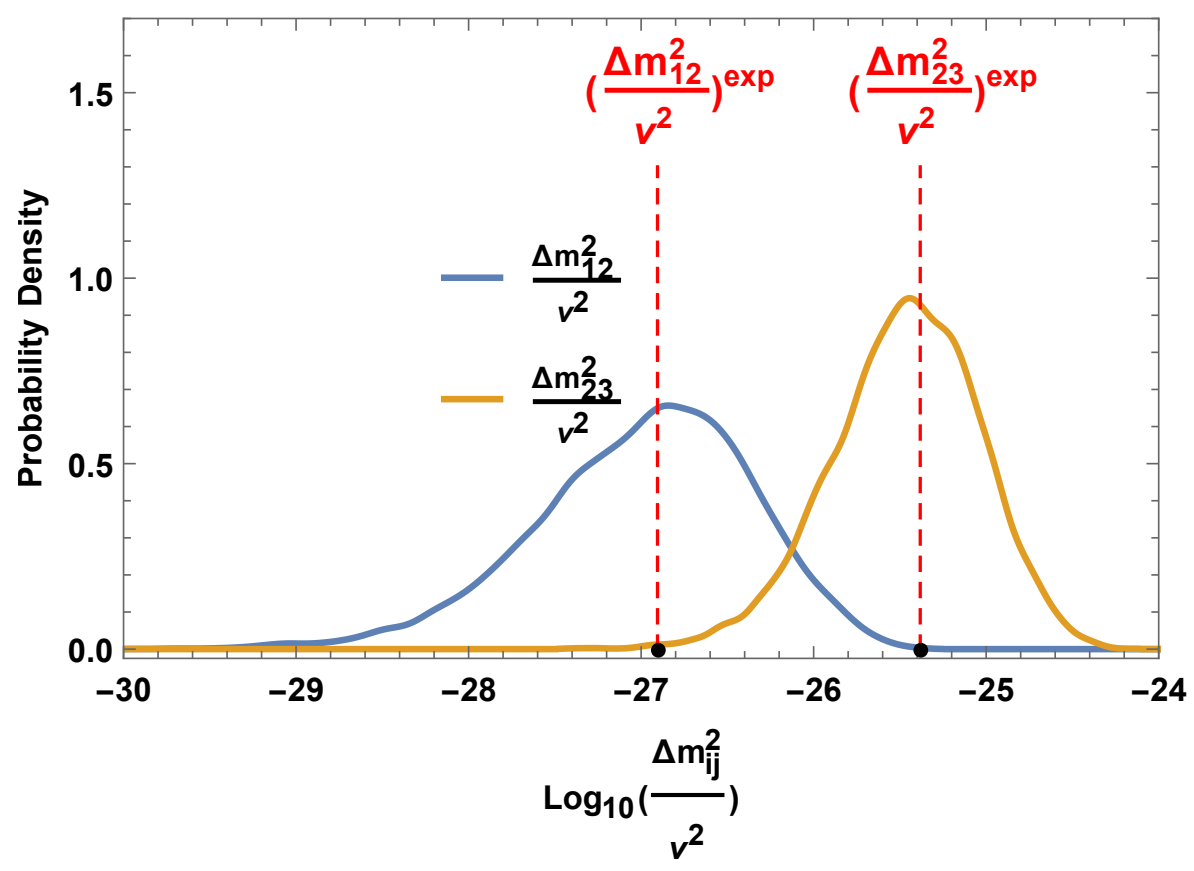

(b) Neutrino squared masses

Figure 2.7: Distributions for the lepton masses under the SM, while considering $\mathcal{O}(1)$ Yukawas. Here it is clear how the charged lepton masses are very unlikely to randomly fall near the experimental lines for the SM configuration. 


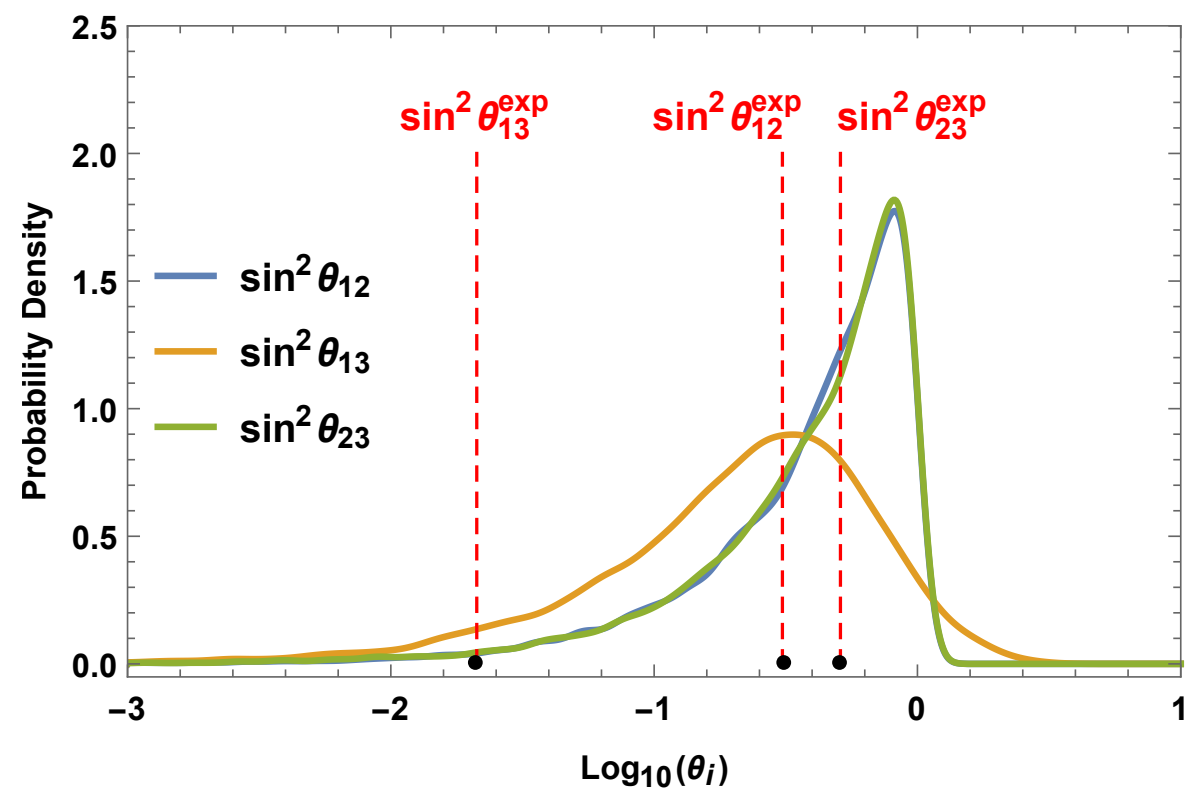

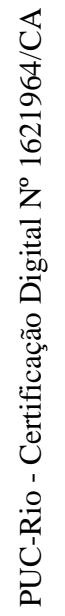

(a) PMNS mixing angles

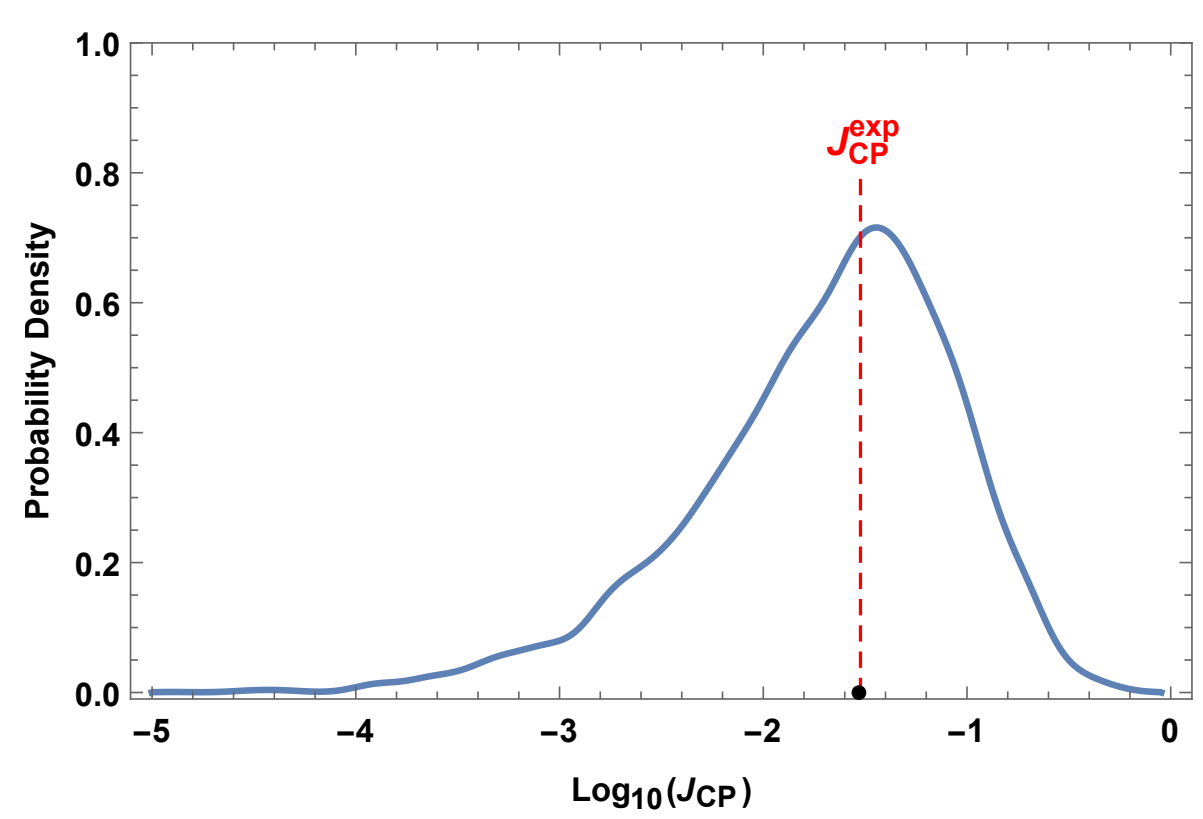

(b) Rephasing invariant

Figure 2.8: Distributions for the lepton mixing parameters under the SM, while considering $\mathcal{O}(1)$ Yukawas. Here, since $\theta_{13}$ is the only small angle, its experimental line ends up falling way off on the distribution curve. 


\section{4}

\section{The path that leads to physics beyond the Standard Model}

The Standard Model has proven to be the most successful theory describing the particles and forces that compose and guide our universe up to date, as it was able to make many powerful predictions that have been beautifully verified experimentally in the last few decades. However, as we have seen, there are still many question that the current theory of the SM is unable to answer. Keeping that in mind, if one wishes to build theories in an attempt to solve the problems which the SM has failed to provide meaningful answers to, it be better be that such theories are also able to replicate all the features contained in the SM.

A textbook way to go beyond the SM (BSM) is by performing an expansion of the Lagrangian in order to be able to account for higher order operators $(\mathcal{O}>4)$. This way, these operators will be suppressed by a cutoff scale $(\Lambda)$, and, at energy scales below $\Lambda$, the theory should be broken down to the Standard Model. This approach is known as Effective Field Theory (EFT) (40), (41).

A Lagrangian under the Effective Field Theory scope can be writen as

$$
\mathcal{L}=\mathcal{L}_{4}+\frac{1}{\Lambda} \mathcal{L}_{5}+\frac{1}{\Lambda^{2}} \mathcal{L}_{6}+\ldots
$$

where $\mathcal{L}_{n}$ is composed only by $\mathcal{O}(n)$ operators and the leading BSM effects are given by $\mathcal{L}_{6}$. And so, one can build higher order Lagrangians out of fields and derivatives, while respecting Lorentz and gauge invariance. Here is important to keep track of the dimension of different fields and derivatives according to Table 2.7.

Usually, the cutoff scale is taken to be above the dynamical scale currently achievable by the experimental technology at the time.

There are infinitely many terms one can build from the operators in Table 2.7, once the usual restriction of dimension four is desregarded, so one has the freedom of picking the best fitted operators for the new physics one is trying to account for, however, it is important to point out that one can safely only consider operators up to dimension-6, as operators of dimension-7 and higher are extremely suppressed and their effects are taken as neglectable. If one would build $\mathcal{O}(5)$ operators out of only the SM fields, the only possible term that is also electroweak invariant would be (42)

$$
\mathcal{L}_{5}=\frac{1}{\Lambda}(\bar{L} \tilde{H})(\tilde{H} L)^{\dagger},
$$

which can generate a Majorana mass 


$$
m_{\nu} \sim \frac{v^{2}}{\Lambda}
$$

where $\Lambda \sim 10^{13} \mathrm{GeV}$ is a typical scale of new physics associated to neutrino masses, whereas there are multiple possible dimension- 6 operators that can be built from SM fields (43). One example is the proton decay, which requires Baryon number (B) violation, and can be writen as (44), (45)

$$
\mathcal{L}_{6}=\frac{1}{\Lambda^{2}}\left(u_{R} d_{R}\right)\left(u_{R} e_{R}\right)+\ldots
$$

where the colour indices are omitted, and if one uses the experimental data for the proton decay lifetime (7), one can estimate $\Lambda>5 \times 10^{16} \mathrm{GeV}$.

The most well known example of a EFT is the Fermi theory, which is the effective low-energy description of the electroweak theory and is able to mimic very accurately the effects of the weak interactions at low energies. This was, historically, the first model that was able to successfully explain the effects of radioactive decay at low energy scales. The muon decay tree-level diagram (Fig. 2.9) can be taken from the Lagrangian in Eq. (2-72) after applying the basis change from Eq. (2-41). This gives

$$
\mathcal{M}=-\left(\frac{i e}{\sqrt{2} \sin \theta}\right)^{2}\left(\bar{e}_{L} \gamma^{\mu} \nu_{e_{L}}+\bar{\mu}_{L} \gamma^{\mu} \nu_{\mu_{L}}\right) \frac{i\left(g^{\mu \nu}-\frac{p^{\mu} p^{\nu}}{m_{W}^{2}}\right)}{p^{2}-m_{W}^{2}}\left(\bar{\nu}_{e_{L}} \gamma^{\nu} e_{L}+\bar{\nu}_{\nu_{L}} \gamma^{\nu} \mu_{L}\right),
$$

where the $W$ propagator is given by

$$
G_{W}^{\mu \nu}=\frac{i\left(g^{\mu \nu}-\frac{p^{\mu} p^{\nu}}{m_{W}^{2}}\right)}{p^{2}-m_{W}^{2}} .
$$

And so, for low energies, one can take the approximation

$$
p^{2} \ll m_{W}^{2},
$$

which breaks down the Feynman diagram to one with a four-fermion interaction, as seen in Fig. 2.9, causing the amplitude in Eq. (2-89) to be approximated to

$$
\mathcal{M}_{4 F}=-\frac{4 G_{F}}{\sqrt{2}}\left(\bar{e}_{L} \gamma^{\mu} \nu_{e_{L}}+\bar{\mu}_{L} \gamma^{\mu} \nu_{\mu_{L}}\right)\left(\bar{\nu}_{e_{L}} \gamma^{\mu} e_{L}+\bar{\nu}_{\nu_{L}} \gamma^{\mu} \mu_{L}\right)
$$

where $G_{F}$ is the Fermi coupling constant and is given by

$$
\frac{4 G_{F}}{\sqrt{2}} \equiv \frac{e^{2}}{2 m_{W}^{2} \sin ^{2}(\theta)}=\frac{2}{v^{2}}
$$

This way, if one considers the dimension of the fields in Table 2.7, it becomes clear in Eq. (2-92) that the four-fermion interaction will yield a dimension6 operator, which constitute the EFT, with the cutoff scale being the Higgs $\mathrm{VEV}, v$. 
In this work, as it will be shown later, we made use of EFT by expanding the clockwork model Lagrangian up to dimension-6 operators in order to be able to compute diagrams containing Flavour Changing Neutral Currents processes. This allowed us to make an experimentally measurable prediction by estimating a lower bound for the typical mass scale of clockwork fermions, which, in the notation of Eq. (2-85), is represented by the scale $\Lambda$.

Table 2.7: Operators that constitute building blocks for theoretical models and their related dimensions in powers of $\hbar$ and length $L$ (46), (47), (48), (49). Here $M, g, \phi, A_{\mu}$ and $\psi$ are a mass scale, a coupling, a scalar field, a vector field and a spinor field, respectively.

\begin{tabular}{cc}
\hline Operator & Dimension \\
\hline$[\mathcal{L}]$ & {$[\hbar] L^{-4}$} \\
{$\left[D_{\mu}\right]$} & $L^{-1}$ \\
{$[M]$} & $L^{-1}$ \\
{$[g]$} & {$[\sqrt{\hbar}]^{-1}$} \\
\hline$[\phi]$ & {$[\sqrt{\hbar}] L^{-1}$} \\
{$\left[A_{\mu}\right]$} & {$[\sqrt{\hbar}] L^{-1}$} \\
{$[\psi]$} & {$[\sqrt{\hbar}] L^{-\frac{3}{2}}$} \\
\hline
\end{tabular}




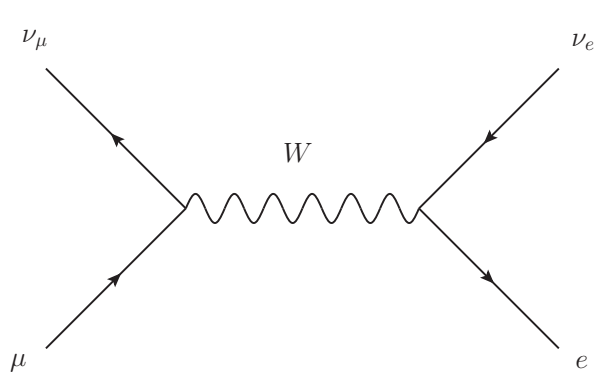

(a) Electroweak interaction mediated by $W$ boson

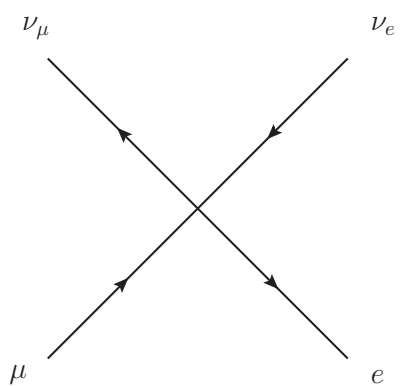

(b) Low energy approximation as four-fermion interaction

Figure 2.9: Feynman diagrams for the muon decay as given by: (a) The SM electroweak theory, where the muon decay is mediated by the $W$ boson and (b) Low energy regime, where the $W$ propagator can be broken down to a single vertex and constitute a dimension-6 four-fermion interaction. 


\section{Froggatt-Nielsen mechanism for mass hierarchy}

One successful approach to explain the fermion masses hierarchy was developed by Froggatt and Nielsen (6). The mechanism works by assuming a flavour $U(1)$ symmetry under which each fermion have different charges. This symmetry is spontaneously broken after a scalar field called "flavon" $(S)$ acquires a VEV. The mass and mixing hierarchies arise via this symmetry breaking from the fact that, since different fermions have different charges under $U(1)_{\text {flavour }}$, each flavour of fermion interacts differently with the flavon field. The procedure involves achieving an EFT by integrating out heavy fermions, called Froggatt-Nielsen fields, which are present only in the full theory, in a energy scale above the flavour dynamics scale, $\Lambda$ (50). In this theory, the hierarchy comes directly from higher order operators, such as:

$$
\mathcal{O}=\frac{S^{n_{i j}}}{\Lambda^{n_{i j}}} \tilde{H} \bar{q}_{L}^{i} u_{R}^{j} y_{i j}^{u} \quad, \quad \mathcal{O}=\frac{S^{n_{i j}}}{\Lambda^{n_{i j}}} H \bar{q}_{L}^{i} d_{R}^{j} y_{i j}^{d} .
$$

Assuming that the flavon has a charge $Q_{S}=-1$ for the $U(1)$ flavour symmetry, in order to have a consistent theory one must impose conservation of charge on the higher order terms above and find a relation between the charges of fermions and the power of the flavon field, $n_{i j}$ :

$$
-n_{i j}+Q_{\bar{q}_{i}}+Q_{u_{j}}=0
$$

In the EFT, after the flavon gets a VEV, different powers of a small parameter

$$
\epsilon=\frac{\langle S\rangle}{\Lambda}
$$

appear in the mass matrices, according to the different charges under the $U(1)$ flavour symmetry, causing the hierarchies explicitly. This effect can be seen on Eq. (3-3).

In this mechanism, the charge assignment varies from different versions of the model and one has the freedom to choose the one found most suitable. Here, we have chosen the charges of the quarks according to Table 3.1 (50). Here we have introduced a free parameter $n$, on the down sector, in order to be able to account for the hierarchy between the up and down sectors, as one 
Table 3.1: Quarks charges assigned for the $U(1)$ flavor symmetry (50). Here, the parameter $n$ is introduced to allow an optimization of the mass hierarchy via computer simulation.

\begin{tabular}{cccc}
\hline & \multicolumn{3}{c}{ Generation } \\
Field & First & Second & Third \\
\hline $\bar{q}_{L}$ & 4 & 2 & 0 \\
$u_{R}$ & 4 & 2 & 0 \\
$d_{R}$ & $2+n$ & $1+n$ & $1+n$ \\
\hline
\end{tabular}

can recall

$$
\frac{y_{t}}{y_{b}} \sim 40
$$

This free parameter will be optimized by allowing us to find, via computer simulation, the value for $n$ that best fit the experimental data for the mass hierarchies.

Now, the Yukawas will be modified by diagonal matrices that have powers of $\epsilon$ as elements. In the case of the up Yukawa, for example:

$$
M_{u}=\left(\begin{array}{ccc}
\epsilon^{4} & & \\
& \epsilon^{2} & \\
& & 1
\end{array}\right)\left(\begin{array}{lll}
y_{11}^{u} & y_{12}^{u} & y_{13}^{u} \\
y_{21}^{u} & y_{22}^{u} & y_{23}^{u} \\
y_{31}^{u} & y_{32}^{u} & y_{33}^{u}
\end{array}\right)\left(\begin{array}{lll}
\epsilon^{4} & & \\
& \epsilon^{2} & \\
& & 1
\end{array}\right) .
$$

Here we will proceed with a illustration of the model with a simplified version of only two families and then, move to the full three family mechanism, under which, simulations were run to determine the parameter $n$ which best fit the experimental measurements and to check if the model at hand is able to reproduce the hierarchical spectra in the fermion sector.

\section{1}

\section{Two family model}

We will begin with the mass matrices and work towards the calculation of the CKM matrix and the Cabibbo angle. After integrating out the heavy fermions, one gets the mass matrices in the quark sector:

$$
\begin{gathered}
M_{u}=\left(\begin{array}{ll}
\epsilon^{2} & \\
& 1
\end{array}\right)\left(\begin{array}{ll}
y_{11}^{u} & y_{12}^{u} \\
y_{21}^{u} & y_{22}^{u}
\end{array}\right)\left(\begin{array}{ll}
\epsilon^{2} & \\
& 1
\end{array}\right), \\
M_{d}=\left(\begin{array}{ll}
\epsilon^{2} & \\
& 1
\end{array}\right)\left(\begin{array}{ll}
y_{11}^{d} & y_{12}^{d} \\
y_{21}^{d} & y_{22}^{d}
\end{array}\right)\left(\begin{array}{ll}
\epsilon^{1+n} & \\
& \epsilon^{1+n}
\end{array}\right),
\end{gathered}
$$

where $y_{i j}^{u}$ and $y_{i j}^{d}$ are the up and down Yukawas, respectively. We can also write these matrices in another form by singular value decomposition: 


$$
M_{u}=U_{L}\left(\begin{array}{cc}
m_{1}^{u} & \\
& m_{2}^{u}
\end{array}\right) U_{R}^{\dagger} \quad, \quad M_{d}=D_{L}\left(\begin{array}{cc}
m_{1}^{d} & \\
& m_{2}^{d}
\end{array}\right) D_{R}^{\dagger},
$$

where $m_{i}^{u}$ and $m_{i}^{d}$ are the mass eigenvalues of the up and down mass matrices, respectively and $U_{L}, U_{R}, D_{L}$ and $D_{R}$ are unitary matrices. We will begin the calculation in the up sector and every step should be analogous for the down sector. The masses can be obtained by taking $M_{u} M_{u}^{\dagger}$ on both equations.

$$
M_{u} M_{u}^{\dagger}=\left(\begin{array}{cc}
\left|y_{12}^{u}\right|^{2} \epsilon^{4}+\mathcal{O}\left(\epsilon^{8}\right) & y_{12}^{u}\left(y_{22}^{u}\right)^{*} \epsilon^{2}+\mathcal{O}\left(\epsilon^{6}\right) \\
\left(y_{12}^{u}\right)^{*} y_{22}^{u} \epsilon^{2}+\mathcal{O}\left(\epsilon^{6}\right) & \left|y_{22}^{u}\right|^{2}+\mathcal{O}\left(\epsilon^{4}\right)
\end{array}\right)
$$

On the other hand, one can write the same matrix from Eq. (3-6) and get:

$$
M_{u} M_{u}^{\dagger}=U_{L}\left(\begin{array}{cc}
\left(m_{1}^{u}\right)^{2} & \\
& \left(m_{2}^{u}\right)^{2}
\end{array}\right) U_{L}^{\dagger} .
$$

Then, one can obtain the mass eigenvalues in a simple manner by taking the determinant and the trace of $M_{u} M_{u}^{\dagger}$ from Eq. (3-7) and Eq. (3-8):

$$
\begin{gathered}
\operatorname{Tr}\left(M_{u} M_{u}^{\dagger}\right)=\left(m_{1}^{u}\right)^{2}+\left(m_{2}^{u}\right)^{2}=\left|y_{22}^{u}\right|^{2}+\mathcal{O}\left(\epsilon^{4}\right), \\
\operatorname{det}\left(M_{u} M_{u}^{\dagger}\right)=\left(m_{1}^{u}\right)^{2}\left(m_{2}^{u}\right)^{2}=\left(y_{11}^{u} y_{22}^{u}-y_{12}^{u} y_{21}^{u}\right)^{2} \epsilon^{8} .
\end{gathered}
$$

Now, one can use the fact that

$$
\left(m_{1}^{u}\right)^{2} \ll\left(m_{2}^{u}\right)^{2}
$$

on Eq. (3-9) and find:

$$
m_{2}^{u}=\left|y_{22}^{u}\right|
$$

which can be used as input on Eq. (3-10) to find:

$$
m_{1}^{u}=\left|\frac{y_{11}^{u} y_{22}^{u}-y_{12}^{u} y_{21}^{u}}{y_{22}^{u}}\right| \epsilon^{4} .
$$

Here, the mass hierarchy is already clear due to the fact that the masses have different powers of the small parameter $\epsilon$, that is $m_{1}^{u} \sim \epsilon^{4}$ and $m_{2}^{u} \sim 1$.

Now one can find the CKM matrix by recalling that

$$
V_{C K M}=U_{L}^{\dagger} D_{L},
$$

where $U_{L}$ and $D_{L}$ are the unitary matrices that diagonalize $M_{u}$ and $M_{d}$, respectively. Being so, the next step in the calculation on the up sector is to find the unitary matrix $U_{L}$, which can be obtained by calculating the eigenvector of $M_{u} M_{u}^{\dagger}$ :

$$
M_{u} M_{u}^{\dagger}\left(\begin{array}{c}
a \\
1
\end{array}\right)=\left|y_{22}^{u}\right|^{2}\left(\begin{array}{c}
a \\
1
\end{array}\right)
$$

which can be rewritten explicitly to: 


$$
\left(\begin{array}{c}
y_{12}^{u}\left(y_{22}^{u}\right)^{*} \epsilon^{2}+\mathcal{O}\left(\epsilon^{4}\right) a \\
\left|y_{22}^{u}\right|^{2}+\mathcal{O}\left(\epsilon^{2}\right) a
\end{array}\right)=\left(\begin{array}{c}
a\left|y_{22}^{u}\right|^{2} \\
\left|y_{22}^{u}\right|^{2}
\end{array}\right)
$$

Here $\left(\begin{array}{c}a \\ 1\end{array}\right)$ is the normalized eigenvector and $a$ is an off-diagonal entry in the CKM matrix $(a \ll 1)$. Solving Eq. (3-15) for $a$ gives

$$
a=\frac{y_{12}^{u}}{y_{22}^{u}} \epsilon^{2}
$$

and so the unitary matrix is given by

$$
U_{L}=\left(\begin{array}{cc}
1 & \frac{y_{12}^{u}}{y_{22}^{u}} \epsilon^{2} \\
-\frac{\left(y_{12}^{u}\right)^{*}}{\left(y_{22}^{u}\right)^{*}} \epsilon^{2} & 1
\end{array}\right)
$$

Here, we have used the fact that the matrix $U_{L}$ is unitary to determine it's second eigenvector.

Now, in the down sector, we can repeat the same steps as above in a completely analogous fashion. Doing so gives

$$
\begin{gathered}
m_{1}^{d}=\frac{\left|y_{11}^{d} y_{22}^{d}-y_{12}^{d} y_{21}^{d}\right|}{\sqrt{\left|y_{21}^{d}\right|^{2}+\left|y_{22}^{d}\right|^{2}}} \epsilon^{3+n}, \\
m_{2}^{d}=\left(\sqrt{\left|y_{21}^{d}\right|^{2}+\left|y_{22}^{d}\right|^{2}}\right) \epsilon^{n+1} .
\end{gathered}
$$

Once again, the hierarchy is clear, since

$$
\frac{m_{1}^{d}}{m_{2}^{d}} \sim \epsilon^{2} \ll 1
$$

Calculating the eigenvectors of $M_{d} M_{d}^{\dagger}$, in a similar fashion as done for the up sector, gives:

$$
D_{L}=\left(\begin{array}{cc}
1 & \frac{y_{11}^{d}\left(y_{21}^{d}\right)^{*}+y_{12}^{d}\left(y_{22}^{d}\right)^{*}}{\left|y_{21}^{d}\right|^{2}+\left|y_{22}^{d}\right|^{2}} \epsilon^{2} \\
-\frac{\left(y_{11}^{d}\right)^{*} y_{21}^{d}+\left(y_{12}^{d}\right)^{*} y_{22}^{d}}{\left|y_{21}^{d}\right|^{2}+\left|y_{22}^{d}\right|^{2}} \epsilon^{2} & 1
\end{array}\right)
$$

One can use the results from Eq. (3-16) and Eq. (3-19) as inputs on Eq. (3-13) to find:

$$
V_{C K M}=\left(\begin{array}{cc}
1 & -\left(\frac{y_{12}^{u}}{y_{22}^{u}}-\frac{y_{11}^{d}\left(y_{21}^{d}\right)^{*}+y_{12}^{d}\left(y_{22}^{d}\right)^{*}}{\left|y_{21}^{d}\right|^{2}+\left|y_{22}^{d}\right|^{2}}\right) \epsilon^{2} \\
\left(\frac{\left(y_{12}^{u}\right)^{*}}{\left(y_{22}^{u}\right)^{*}}-\frac{\left(y_{11}^{d}\right)^{*} y_{21}^{d}+\left(y_{12}^{d}\right)^{*} y_{22}^{d}}{\left|y_{21}^{d}\right|^{2}+\left|y_{22}^{d}\right|^{2}}\right) \epsilon^{2} & 1
\end{array}\right) .
$$

The Cabibbo angle is given by:

$$
\theta_{c}=\left(\frac{y_{12}^{u}}{y_{22}^{u}}-\frac{y_{11}^{d}\left(y_{21}^{d}\right)^{*}+y_{12}^{d}\left(y_{22}^{d}\right)^{*}}{\left|y_{21}^{d}\right|^{2}+\left|y_{22}^{d}\right|^{2}}\right) \epsilon^{2}
$$

It is worth noting that, by taking a look at Eq. (3-20), one can clearly see that 
the CKM matrix does not have any dependence on the parameter $n$ and the same will be true for the three family model. This means that such parameter introduced does not affect the mixing in the quark sector in any way, as it only function as a shift in the distribution of the down Yukawa eingevalues $y_{d}, y_{s}$, $y_{b}$.

\section{2}

\section{Three family model}

The calculations for the three family model, although more laborious, are completely analogous. It is worth noting that, while proceeding with the calculations in the three family model, one should only take approximations of the leading order of $\epsilon$ as the last step, otherwise the result might be off. The mass matrices for the quark sector are given by:

$$
\begin{gathered}
M_{u}=\left(\begin{array}{lll}
\epsilon^{4} & & \\
& \epsilon^{2} & \\
& & 1
\end{array}\right)\left(\begin{array}{lll}
y_{11}^{u} & y_{12}^{u} & y_{13}^{u} \\
y_{21}^{u} & y_{22}^{u} & y_{22}^{u} \\
y_{31}^{u} & y_{32}^{u} & y_{33}^{u}
\end{array}\right)\left(\begin{array}{lll}
\epsilon^{4} & & \\
& \epsilon^{2} & \\
& & 1
\end{array}\right), \\
M_{d}=\left(\begin{array}{lll}
\epsilon^{4} & & \\
& \epsilon^{2} & \\
& & \\
& &
\end{array}\right)\left(\begin{array}{lll}
y_{11}^{d} & y_{12}^{d} & y_{13}^{d} \\
y_{21}^{d} & y_{22}^{d} & y_{22}^{d} \\
y_{31}^{d} & y_{32}^{d} & y_{33}^{d}
\end{array}\right)\left(\begin{array}{lll}
\epsilon^{2+n} & & \\
& & \\
& & \\
& & \epsilon^{1+n}
\end{array}\right) .
\end{gathered}
$$

For this configuration, the mass eigenvalues are the following:

$$
\begin{aligned}
& m_{1}^{u}=\left|\frac{y_{11}^{u} y_{22}^{u} y_{33}^{u}+y_{12}^{u} y_{23}^{u} y_{31}^{u}+y_{13}^{u} y_{32}^{u} y_{21}^{u}-y_{12}^{u} y_{21}^{u} y_{33}^{u}-y_{13}^{u} y_{31}^{u} y_{22}^{u}-y_{23}^{u} y_{32}^{u} y_{11}^{u}}{y_{22}^{u} y_{33}^{u}-y_{23}^{u} y_{32}^{u}}\right| \epsilon^{8}, \\
& m_{2}^{u}=\left|\frac{y_{22}^{u} y_{33}^{u}-y_{23}^{u} y_{32}^{u}}{y_{33}^{u}}\right| \epsilon^{4} \\
& m_{3}^{u}=\left|y_{33}^{u}\right| \\
& m_{1}^{d}=\left|\frac{y_{11}^{d} y_{22}^{d} y_{33}^{d}+y_{12}^{d} y_{23}^{d} y_{31}^{d}+y_{13}^{d} y_{32}^{d} y_{21}^{d}-y_{12}^{d} y_{21}^{d} y_{33}^{d}-y_{13}^{d} y_{31}^{d} y_{22}^{d}-y_{23}^{d} y_{32}^{d} y_{11}^{d}}{y_{22}^{d} y_{33}^{d}-y_{23}^{d} y_{32}^{d}}\right| \epsilon^{6+n}, \\
& m_{2}^{d}=\frac{\left|y_{22}^{d} y_{33}^{d}-y_{23}^{d} y_{32}^{d}\right|}{\sqrt{\left|y_{32}^{d}\right|^{2}+\left|y_{33}^{d}\right|^{2}}} \epsilon^{3+n} \\
& m_{3}^{d}=\left(\sqrt{\left|y_{32}^{d}\right|^{2}+\left|y_{33}^{d}\right|^{2}}\right) \epsilon^{1+n} \\
& U_{L}=\left(\begin{array}{ccc}
1 & \frac{y_{12}^{u} y_{33}^{u}-y_{13}^{u} y_{32}^{u}}{y_{22}^{u} y_{33}^{u}-y_{23}^{u} y_{32}^{u}} \epsilon^{2} & \frac{y_{13}^{u}}{y_{33}^{u}} \epsilon^{4} \\
-\frac{y_{12}^{u} y_{33}^{u}-y_{13}^{u} y_{32}^{u}}{y_{22}^{u} y_{33}^{u}-y_{23}^{u} y_{32}^{u}} \epsilon^{2} & 1 & \frac{y_{23}^{u}}{y_{33}^{u}} \epsilon^{2} \\
\frac{y_{12}^{u} y_{23}^{3}-y_{13}^{u} y_{22}^{u}}{y_{22}^{u} y_{33}^{u}-y_{23}^{u} y_{32}^{u}} \epsilon^{4} & -\frac{y_{23}^{u}}{y_{33}^{u}} \epsilon^{2} & 1
\end{array}\right) \text {, } \\
& D_{L}=\left(\begin{array}{ccc}
1 & \frac{y_{12}}{y_{22}^{d} y_{33}^{d}-y_{13}^{d} y_{32}^{d}} \epsilon^{4} & \frac{y_{12}^{d} y_{32}^{d}+y_{13}^{d} y_{33}^{d}}{\left(y_{32}^{u}\right)^{2}+\left(y_{33}^{u} \epsilon^{2}\right.} \epsilon^{6} \\
-\frac{y_{12}^{d} y_{33}^{d}-y_{13}^{d} y_{32}^{d}}{y_{22}^{d} y_{33}^{d}-y_{23}^{d} y_{32}^{d}} \epsilon^{4} & 1 & \frac{y_{22}^{d} y_{32}^{d}+y_{23}^{d} y_{33}^{d}}{\left(y_{32}^{u}\right)^{2}+\left(y_{33}^{u}\right)^{2}} \epsilon^{4} \\
\frac{y_{12}^{d} y_{23}^{d}-y_{13}^{d} y_{22}^{d}}{y_{22}^{d} y_{33}^{d}-y_{23}^{d} y_{32}^{d}} \epsilon^{6} & -\frac{y_{22}^{d} y_{32}^{d}+y_{23}^{d} y_{33}^{d}}{\left(y_{32}^{u}\right)^{2}+\left(y_{33}^{u}\right)^{2}} \epsilon^{4} & 1
\end{array}\right)
\end{aligned}
$$


And the CKM matrix is obtained by taking

$$
V_{C K M}=U_{L}^{\dagger} D_{L}
$$

from Eq. (3-29) and Eq. (3-30). Although the expression for $V_{C K M}$ is not being shown explicitly here, it is very useful to see how it's entries goes as powers of $\epsilon$ :

$$
V_{C K M}^{F N} \sim\left(\begin{array}{ccc}
1 & \epsilon^{2} & \epsilon^{4} \\
\epsilon^{2} & 1 & \epsilon^{2} \\
\epsilon^{4} & \epsilon^{2} & 1
\end{array}\right),
$$

where $F N$ stands for Froggatt-Nielsen. In this way we can compare the CKM matrix built from the Froggatt-Nielsen mechanism to the one measured experimentally (7) and come up with an estimation for the parameter $\epsilon$.

$$
V_{C K M}^{\exp } \sim\left(\begin{array}{ccc}
1 & 0.2 & 0.004 \\
0.2 & 1 & 0.04 \\
0.008 & 0.04 & 1
\end{array}\right)
$$

This way, one can come up with a estimation for

$$
\epsilon \sim 0.2
$$

even before running the simulations to determine the value of $\epsilon$ that gives the optimal distributions for the entries in the Froggatt-Nielsen CKM matrix when compared to the experimentally measured CKM matrix.

Additionally, one can take a look at Eq. (3-32) and compare it to the standard parametrization of the CKM matrix to come up with the following relation for the mixing angles produced via the mechanism:

$$
\theta_{i j} \sim \epsilon^{Q_{q_{i}}-Q_{q_{j}}}, \quad \text { for } i<j,
$$

where $Q_{q_{i}}$ is the $U(1)_{\text {flavour }}$ charge of the doublet $q_{i}$ as seen on Table 3.1. Thus, one should expect the results of the simulations to yield

$$
\theta_{12} \sim \epsilon^{2}, \quad \theta_{23} \sim \epsilon^{2}, \quad \theta_{13} \sim \epsilon^{4} .
$$

This way, the distributions for $\theta_{12}$ and $\theta_{23}$ should be similar, while the distribution for $\theta_{13}$ should lie in a lower spectrum.

\subsection{1}

\section{Simulations for the three family model}

The goal of these simulations is to find the value of the parameter $\epsilon$ that best fit the experimental data available for the CKM matrix entries, while also finding the best value for the free parameter $n$, regarding the flavour symmetry charge assignment in the down sector (see Table 3.1), that fits the best with 
the experimentally measured mass hierarchy present in the down sector. This can be achieved by making use of the $\chi^{2}$ test, which consist in finding the value for $\epsilon$ that yields the minimum value for the quantity

$$
\chi^{2}=\sum_{\substack{i \neq j \\ k \neq l}}^{3} \frac{\left(\left|V_{i j}^{e x p}\right|-\epsilon^{c_{i j}}\left\langle\left|V_{i j}\right|\right\rangle\right)}{\sigma_{\left|V_{i j}\right|}} C_{i j, k l} \frac{\left(\left|V_{k l}^{e x p}\right|-\epsilon^{c_{k l}}\left\langle\left|V_{k l}\right|\right\rangle\right)}{\sigma_{\left|V_{k l}\right|}}
$$

where $\left|V_{i j}^{e x p}\right|$ is the experimental absolute value for the $i j$-entry in the CKM matrix, $\left|V_{i j}\right|$ is the corresponding simulated distribution, $\sigma_{\left|V_{i j}\right|}$ is the standard deviation of such distribution, $C_{i j}$ is the correlation matrix between $\left|V_{i j}\right|$ and $\left|V_{k l}\right|$, and $c_{i j}$ is the corresponding power of $\epsilon$ that the $i j$-entry in the Froggatt-Nielsen CKM matrix exhibits, as seen in Eq. (3-32) (for example, since $\left(V_{C K M}^{F N}\right)_{12} \sim \epsilon^{2}$, then on has $c_{i j}=2$ ).

Then the parameter $\epsilon$ is determined by solving the equation

$$
\frac{\partial}{\partial c_{i}} \chi^{2}(\epsilon)=0
$$

which guarantees the minimum value for $\chi^{2}$, by taking

$$
\chi_{\min }^{2}=\chi^{2}\left(\epsilon^{o p t}\right),
$$

where $\epsilon^{\text {opt }}$ is the solution for Eq. (3-38).

From here, we proceeded with a ten thousand run simulation, while assuming natural Yukawas with random $\mathcal{O}(1)$ entries with a uniform distribution, that is

$$
0 \leq\left|(Y)_{i j}\right| \leq 1, \quad \text { for } i, j=1,2,3,
$$

for both the down and up sectors. This way, one can determine the distributions for the entries of the CKM matrix, the mixing angles and the Yukawas in the quark sector, while also finding the solution for Eq. (3-38) as well as the optimal value for $n$. The best fit values for $\epsilon$ and $n$, as well as the optimized value for $\chi^{2}$, via this mechanism, are presented in Table 3.3, while the experimental values of the relevant quantities are shown in Table 3.2 taken from (7) and Eq.(3-40).

The distribution for the Yukawas on the quark sector can be seen in Fig. 3.1, while the distribution for the mixing angles and the Jarlskog invariant are shown in Fig. 3.2. It is easy to see that the results for all the distributions agree very well with the experimental data and, in that sense, the mechanism can be regarded as successful as it produces consistent results, specially due to the fact that if one would run a simulation in the SM to determine the distribution of the same quantities (by making use of the same assumption 
that $\left.0 \leq\left|(Y)_{i j}\right| \leq 1\right)$ the corresponding distributions would be way off the experimental value lines.

Additionally, one can see in Table 3.3 that the value for $\epsilon$ obtained via the simulation matches very well with our initial estimation done in Eq. (3-34). Another result that confirms our expectations, comes from the distribution of the mixing angles $\theta_{i j}$, as one can see in Fig 3.2, that it matches fine with the relation obtained from Eq. (3-36).

Table 3.2: Quark sector variables and their experimental values taken from (7).

$$
\begin{aligned}
& \begin{array}{cc}
\hline \text { Quantity } & \text { Experimental value } \\
\hline y_{d} & 1.364 \times 10^{-5}
\end{array} \\
& y_{s} \quad 2.70 \times 10^{-4} \\
& \begin{array}{cc}
y_{b} & 1.388 \times 10^{-2} \\
\hline y_{u} & 6.3 \times 10^{-6}
\end{array} \\
& y_{c} \quad 3.104 \times 10^{-3} \\
& \begin{array}{cc}
y_{t} & 0.8685 \\
\hline \sin ^{2}\left(\theta_{12}\right) & 0.307
\end{array} \\
& \sin ^{2}\left(\theta_{23}\right) \quad 0.51 \\
& \sin ^{2}\left(\theta_{13}\right) \quad 2.12 \times 10^{-2} \\
& J \quad 3.18 \times 10^{-5} \\
& \begin{aligned}
\left|V_{C K M}\right| & =\left(\begin{array}{ccc}
\left|V_{u d}\right| & \left|V_{u s}\right| & \left|V_{u b}\right| \\
\left|V_{c d}\right| & \left|V_{c s}\right| & \left|V_{c b}\right| \\
\left|V_{t d}\right| & \left|V_{t s}\right| & \left|V_{t b}\right|
\end{array}\right) \\
& =\left(\begin{array}{ccc}
0.97420 & 0.2243 & 3.94 \times 10^{-3} \\
0.218 & 0.997 & 42.2 \times 10^{-3} \\
8.1 \times 10^{-3} & 39.4 \times 10^{-3} & 1.019
\end{array}\right) .
\end{aligned}
\end{aligned}
$$

Table 3.3: Quark sector best fit free parameters for the Froggatt-Nielsen mechanism. The value for $\epsilon$ corresponds to the initial expectation, as seen in (50), while the low value for $\chi_{\min }^{2}$ (taking into account the six degrees of freedom considered) is clear evidence for the fact that the model agrees very well with the experimental data.

\begin{tabular}{cc}
\hline Quantity & Value \\
\hline$\epsilon$ & 0.24 \\
$n$ & 2 \\
\hline$\chi_{\min }^{2}$ & 3.81 \\
\hline
\end{tabular}




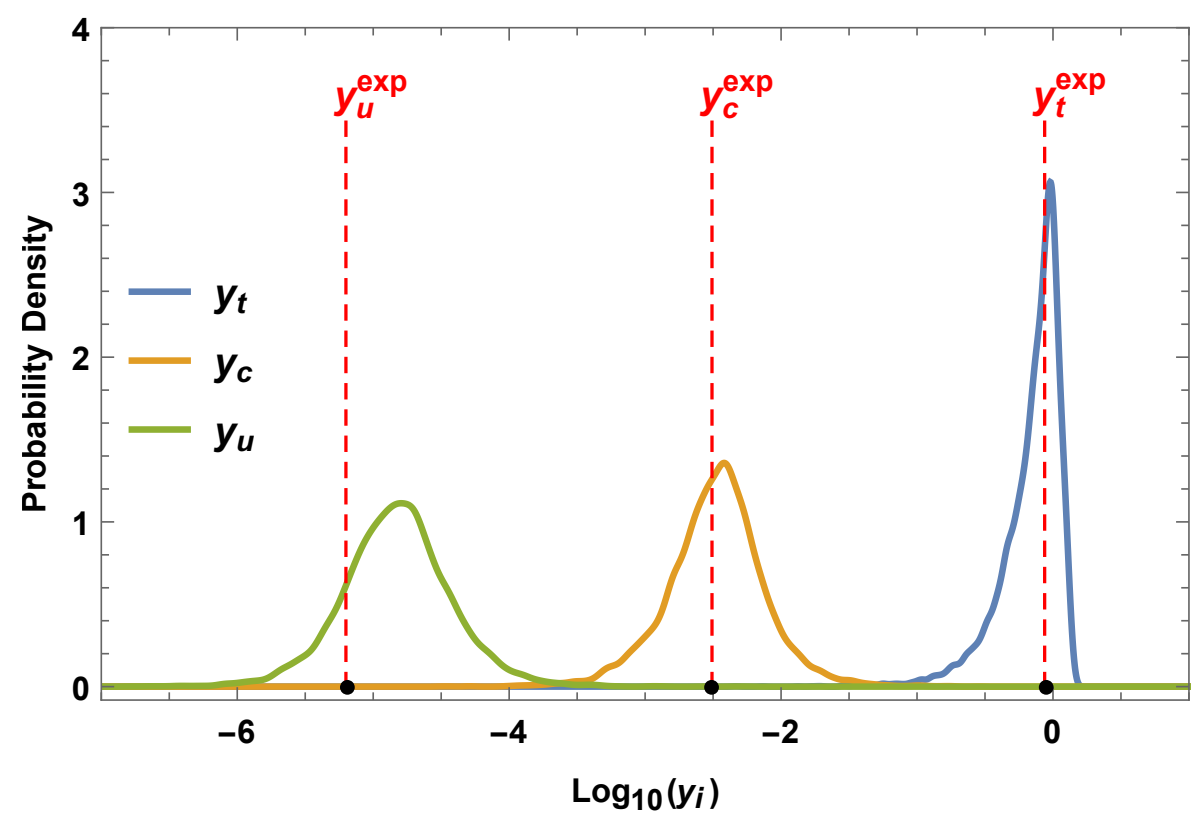

(a) Up sector masses

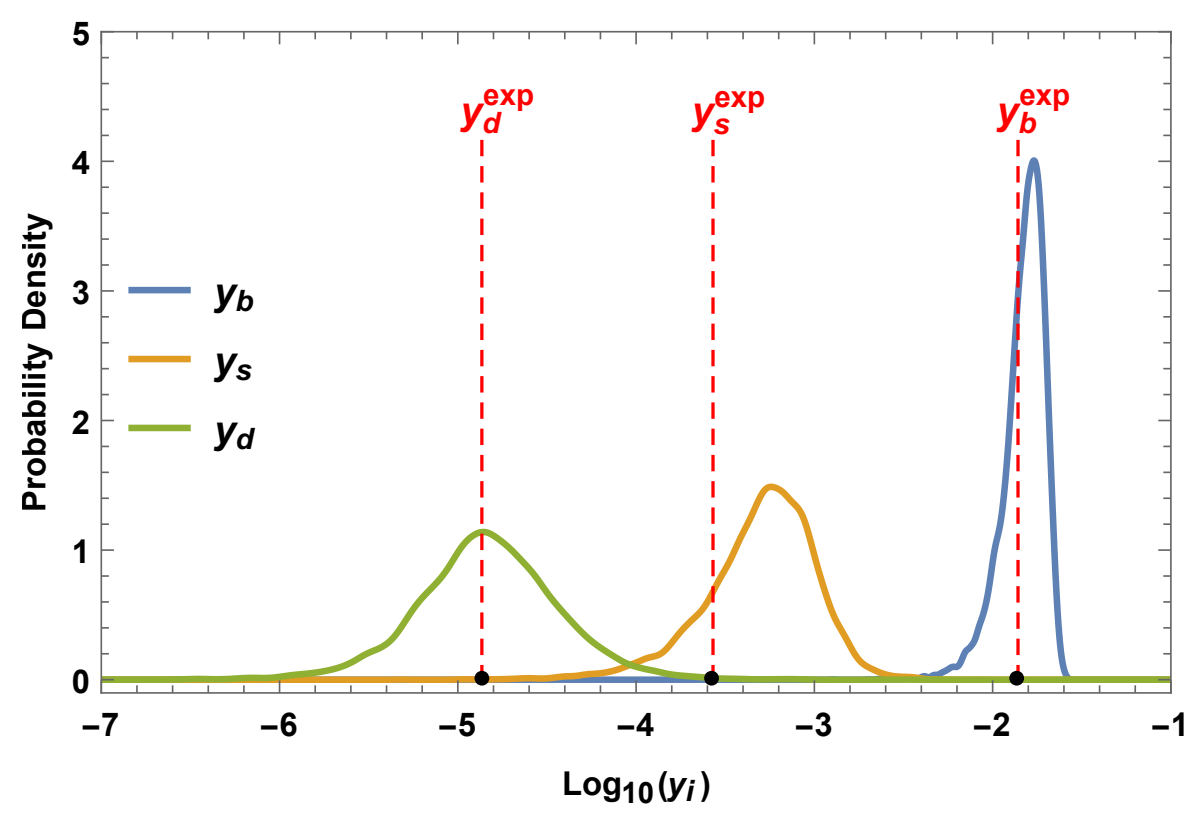

(b) Down sector masses

Figure 3.1: Quark masses distributions for the Froggatt-Nielsen mechanism. One can clearly see that the distributions match perfectly with the experimental values, when the optimized value of $n=2$ is used. 


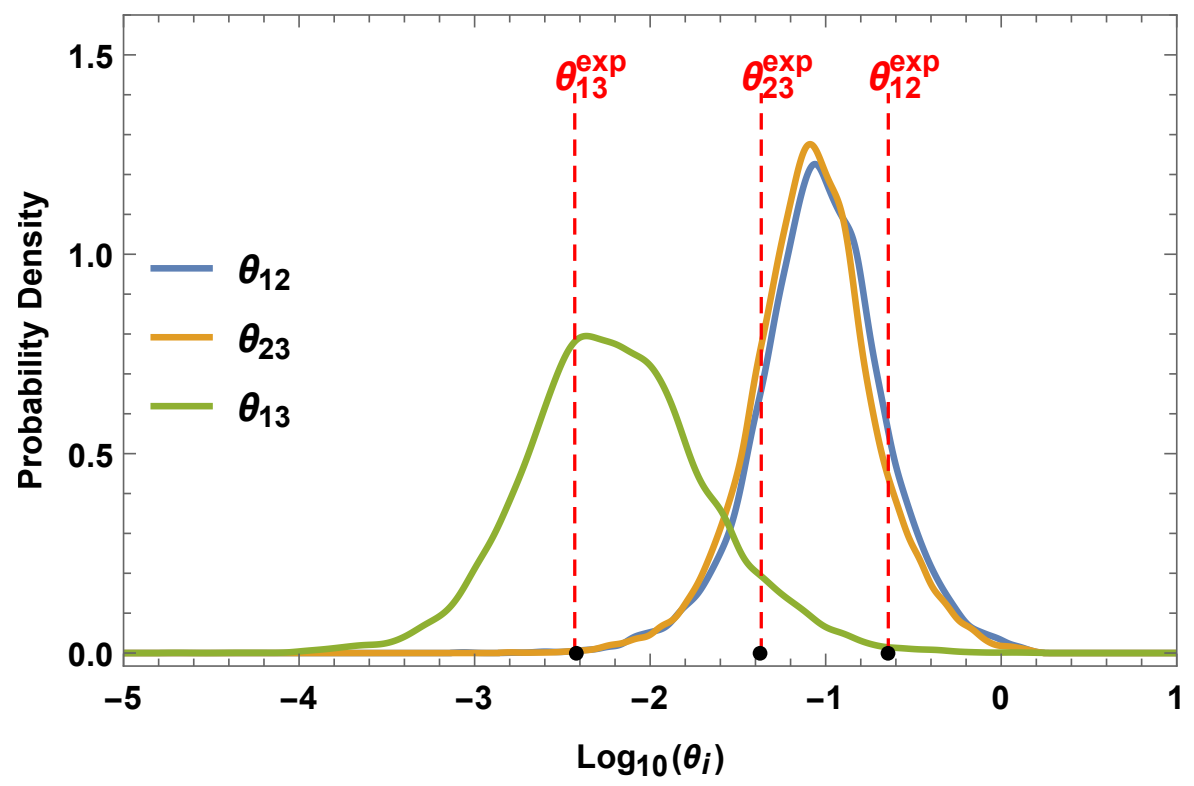

(a) CKM mixing angles

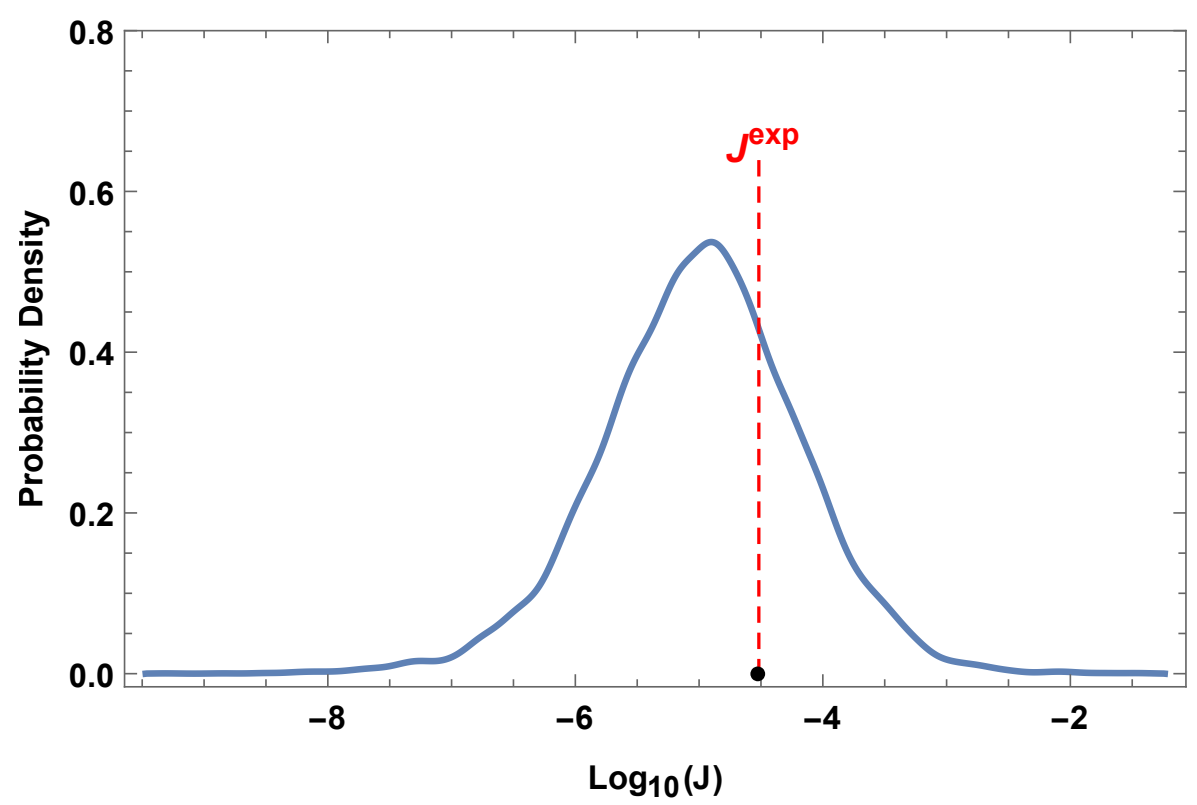

(b) Jarlskog invariant

Figure 3.2: Quark mixing distributions for the Froggatt-Nielsen mechanism. The experimental values seem to fit greatly within the distributions. As it was expected, from Eq. (3-36), the distributions for $\theta_{12}$ and $\theta_{23}$ are very similar. 


\section{4}

\section{Clockwork Model}

Originally designed as a solution to a certain problem of the relaxion mechanism (51), (52), this model was later generalized for different systems (12) and became a powerful theory building tool for generating natural small numbers out of $\mathcal{O}(1)$ parameters (15). Different applications of the model reaching beyond the scope of the flavour hierarchy problem can be found in (53), (54), (55), (56), (57), (58), (59), (60), (61), (62), (63), (64), (65), (66) and (67).

The generalized setup starts with a particle $P$ that is taken as massless due to a symmetry $\mathcal{S}$. The next step is to make $N+1$ copies of the original particle, and take them as sites in a one-dimensional lattice in theory space. These particles are called the 'clockwork gears', as they are the degrees of freedom that make the mechanism work. The lattice is taken as to have a $\mathcal{G}$ symmetry which contains at least the product of the symmetries from each site, that is, $\mathcal{G} \supseteq \mathcal{S}^{N+1}$. The procedure is to introduce a soft breaking of the $\mathcal{G}$ symmetry, at a mass scale $m$, caused by a mass mixing term between each immediate neighbour for each site. Additionally, a parameter $q \neq 1$ is also introduced. This parameter acts on the lattice in an asymmetric fashion, as it treats the $j$ and $j+1(j=0,1, \ldots, N-1)$ sites differently. Since we are working with a one-dimensional lattice with boundaries, the setup must contain $N+1$ sites and $N$ links. This way, as each link breaks a symmetry in a single site, one symmetry group $\mathcal{S}^{\prime}$ is bound to remain after the breaking. Therefore, we end up with a single massless particle $P^{\prime}$ (zero mode) that can be viewed as a linear combination of the original particles, that is:

$$
P^{\prime}=\sum_{i=0}^{N} c_{i} P_{i}
$$

The power of the model comes from the fact that the distribution of the particle $P^{\prime}$ along the sites in theory space turns out to not be uniform, but rather exponential, localising $P^{\prime}$ towards one of the boundaries. Another way to look at this is to realize that, as we will see explicitly later when we build a theory for the clockwork fermion, the components of $P^{\prime}$ happens to be $c_{i} \sim q^{2 i}$, pushing $P^{\prime}$ much closer to one of the boundaries. This effect works greatly in our favour as it allows us to achieve a exponentially suppressed component of $P^{\prime}$ at the 
opposite boundary, for sufficiently large values of $N$. In that sense, if we couple the SM to the clockwork sector at this boundary, we automatically obtain very small couplings to $P^{\prime}$, without the need to introduce small parameters. That is the main strength of the model, being able to naturally generate suppressed interactions.

It is also important to point out that another positive trait of the model is the fact that it has verifiable predictions which can be experimentally checked. The model predicts the existence of $N$ particles, at a mass scale $m$, that interacts weakly to the SM in a predictable manner according to the mechanism implementation at hand. The model can be applied for spin-0 scalars, spin- $\frac{1}{2}$ fermions, spin-1 bosons and spin- 2 gravitons. In this thesis we will apply the mechanism to spin- $\frac{1}{2}$ fermions in order to propose a solution to the fermion mass hierarchy problem.

\section{1}

\section{Clockwork fermion}

In the case where particle $P$ is a fermion, the aforementioned symmetry $\mathcal{S}$ that keeps the particle massless can be a chiral symmetry. We then introduce $N+1$ right handed chiral fermions $\psi_{R}^{j}(j=0,1, \ldots, N)$, which remain massless due to a $U(1)_{R_{j}}$ symmetry, and $N$ left handed chiral fermions $\psi_{L}^{j}$ $(j=0,1, \ldots, N-1)$, which are masless due to a $U(1)_{L_{j}}$ symmetry. This way, when we apply the soft breaking parameters, a single chiral symmetry will survive, exponentially pushing the chiral massless fermion towards one of the boundaries. In order to achieve this, we take the mass mixing parameter $m_{j}$ to pair up fields of opposite chiralities, forming this way $N$ Dirac mass terms $\left(m_{j} \bar{\psi}_{L}^{j} \psi_{R}^{j}\right)$. We also bring in a $k_{j}=(m q)_{j}$ factor to act on neighbour fields of opposite chiralities composing $N$ terms like $k_{j} \bar{\psi}_{L}^{j} \psi_{R}^{j+1}$. Both of these parameters can be interpreted as spurion fields that acquired VEVs as a consequence of spontaneously broken symmetries at higher energy scales. The charges assigned to the fields and parameters are presented in Table 4.1.

Table 4.1: Charges for the clockwork fermion under different symmetries. The charges are assigned in such a way that one can build invariant terms as $m_{j} \bar{\psi}_{L}^{j} \psi_{R}^{j}$ and $k_{j} \bar{\psi}_{L}^{j} \psi_{R}^{j+1}$.

\begin{tabular}{lrll}
\hline & \multicolumn{3}{c}{ Charges } \\
\hline$\psi_{R}^{j}$ & 1 & under & $U(1)_{R_{j}}$ \\
$\psi_{L}^{j}$ & 1 & under & $U(1)_{L_{j}} \times U(1)_{R_{j}}$ \\
$m_{j}$ & $(1,-1)$ & under & $U(1)_{L_{j}} \times U(1)_{R_{j+1}}$ \\
$k_{j}$ & $(1,-1)$ & under & $U(1)_{L_{j}} \times U\left({ }^{2}\right.$ \\
\hline
\end{tabular}

With this charge configuration we can be build terms in the Lagrangian which 
are invariant under the symmetries above, such as:

$$
\Delta \mathcal{L}_{1}=m_{j} \bar{\psi}_{L}^{j} \psi_{R}^{j} \quad, \quad \Delta \mathcal{L}_{2}=k_{j} \bar{\psi}_{L}^{j} \psi_{R}^{j+1} .
$$

It is very helpful to visualize the general configuration of the lattice with Fig. 4.1 , in order to get a deeper understanding of the mechanism at work:

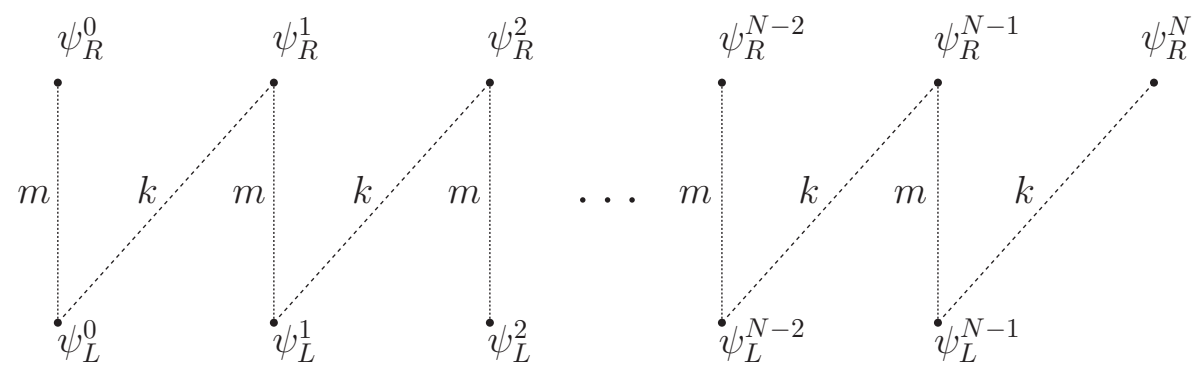

Figure 4.1: Lattice configuration of the clockwork mechanism for fermions. Here the parameters $m$ and $k$ are taken as universal in order to simplify the model.

Where we have taken a simplified mechanism in which $m$ and $k$ are universal across every possible pair of fields. It is clear from this configuration that after the introduction of the symmetry breaking via the mass mixing term, a single field remain massless. which, in this case, was chosen to be the boundary fermion $\psi_{R}^{N}$.

So the here goal is to couple the SM to this massless fermion in order to obtain exponentially suppressed interactions, without the need to introduce small parameters. By this procedure we can achieve a fermion hierarchy in a more natural fashion. The clockwork fermion Lagrangian will look like:

$$
\mathcal{L}_{N}=\sum_{j=0}^{N} i \bar{\psi}_{R}^{j} \not D \psi_{R}^{j}+\sum_{j=0}^{N-1} i \bar{\psi}_{L}^{j} \not D \psi_{L}^{j}-m \sum_{j=0}^{N-1}\left(\bar{\psi}_{L}^{j} \psi_{R}^{j}-q \bar{\psi}_{L}^{j} \psi_{R}^{j+1}+\text { h.c. }\right),
$$

where again, we have taken $m$ and $k$ to be universal in order to simplify the calculations. To find masses (and Yukawa couplings) of the zero mode one could diagonalize the full fermion mass matrix, as originally done in (12), (68) and (69). Here instead, we will use the technique of an interpolating field (chosen to be $\psi_{R}^{N}$ ) as explained in the following. One can exhibit the effects of the clockwork mechanism by means of integrating out all the fields and write out a new Lagrangian in terms of only $\psi_{R}^{N}$. To easily demonstrate this process, we will start with the simplest case where we take $N=1$. Thus our Lagrangian will be:

$$
\begin{array}{r}
\mathcal{L}_{1}=i \bar{\psi}_{L}^{0} \not \supset \psi_{L}^{0}+i \bar{\psi}_{R}^{0} \not D \psi_{R}^{0}+i \bar{\psi}_{R}^{1} \not \supset \psi_{R}^{1}-m\left(\bar{\psi}_{L}^{0} \psi_{R}^{0}-q \bar{\psi}_{L}^{0} \psi_{R}^{1}+\bar{\psi}_{R}^{0} \psi_{L}^{0}-q \bar{\psi}_{R}^{1} \psi_{L}^{0}\right) \\
+\bar{\psi}_{R}^{1} \mathcal{O}+\overline{\mathcal{O}} \psi_{R}^{1}, \quad(4-4)
\end{array}
$$


where we have introduced an operator $\mathcal{O}$ to represent the fields that one might wish to couple via the mechanism (e.g. Yukawa), this way it will be easier to see later why such coupling can be suppressed. To integrate the unwanted fields, we ought to apply the equations of motion for each of the following fields: $\bar{\psi}_{L}^{0}$, $\bar{\psi}_{R}^{0}$. Therefore, after we get rid of those fields, the only terms remaining will be the ones with the $\psi_{R}^{1}$ field. Using the Euler-Lagrange equations

$$
\frac{\partial \mathcal{L}}{\partial \psi}-\partial_{\mu} \frac{\partial \mathcal{L}}{\partial\left(\partial_{\mu} \psi\right)}=0
$$

first, we integrate out $\psi_{L}^{0}$ :

$$
\not p \psi_{L}^{0}-m\left(\psi_{R}^{0}-q \psi_{R}^{1}\right)=0 \quad \Longrightarrow \quad \psi_{L}^{0}=\frac{m}{\not p}\left(\psi_{R}^{0}-q \psi_{R}^{1}\right)
$$

putting this result back in the Lagrangian gives us:

$$
\mathcal{L}_{1}=\bar{\psi}_{R}^{0} \not p \psi_{R}^{0}+\bar{\psi}_{R}^{1} \not p \psi_{R}^{1}-m\left(\bar{\psi}_{R}^{0}-q \bar{\psi}_{R}^{1}\right) \frac{m}{\not p}\left(\psi_{R}^{0}-q \psi_{R}^{1}\right)+\bar{\psi}_{R}^{1} \mathcal{O}+\overline{\mathcal{O}} \psi_{R}^{1}
$$

Now, integrating out $\psi_{R}^{0}$ :

$$
\not p \psi_{R}^{0}-\frac{m^{2}}{\not p}\left(\psi_{R}^{0}-q \psi_{R}^{1}\right)=0 \quad \Longrightarrow \quad \psi_{R}^{0}=\frac{m^{2} q}{m^{2}-p^{2}} \psi_{R}^{1}
$$

and returning this result to the previous Lagrangian let us finally write our Lagrangian in terms of only $\psi_{R}^{1}$ :

$$
\mathcal{L}_{1}=\bar{\psi}_{R}^{1} \not p\left[1+\frac{m^{2} q^{2}}{p^{2}}\left(\frac{m^{2}}{m^{2}-p^{2}}-1\right)\right] \psi_{R}^{1}+\bar{\psi}_{R}^{1} \mathcal{O}+\overline{\mathcal{O}} \psi_{R}^{1},
$$

which can be rewritten as:

$$
\mathcal{L}_{1}=\bar{\psi}_{R}^{1} \not p\left[1+\frac{m^{2} q^{2}}{p^{2}}\left(\frac{1}{1-\frac{p^{2}}{m^{2}}}-1\right)\right] \psi_{R}^{1}+\bar{\psi}_{R}^{1} \mathcal{O}+\overline{\mathcal{O}} \psi_{R}^{1} .
$$

However, if we take the soft limit, where $p^{2} \ll m^{2}$, we can use the approximation:

$$
\frac{1}{1-\frac{p^{2}}{m^{2}}} \approx 1+\frac{p^{2}}{m^{2}}
$$

This allow us to write our final elegant Lagrangian:

$$
\mathcal{L}_{1}=\bar{\psi}_{R}^{1} \not p\left(1+q^{2}\right) \psi_{R}^{1}+\bar{\psi}_{R}^{1} \mathcal{O}+\text { h.c. },
$$

which can be rewritten by performing the transformation

$$
\psi_{R}^{1} \rightarrow \frac{1}{\sqrt{1+q^{2}}} \psi_{R}^{1}
$$


in order to achieve a canonical kinetic term. In this way, one ends up with

$$
\mathcal{L}_{1}=i \bar{\psi}_{R}^{1} \not \partial \psi_{R}^{1}+\frac{1}{\sqrt{1+q^{2}}} \bar{\psi}_{R}^{1} \mathcal{O}+\text { h.c. . }
$$

Here one might have $q \ll 1$ and the factor $1+q^{2} \approx 1$, indicating that the zero mode field has $\mathcal{O}(1)$ mixing with $\psi_{R}^{1}$ and is then located near the $N=1$ site. On the other hand, if $q \gg 1$ the factor $1+q^{2} \gg 1$, leading to suppressed couplings of the zero mode, indicating small mixing with $\psi_{R}^{1}$ and a localization towards the opposite boundary. This thought process is summarized in Table 4.2. Alternatively, one could have chosen $\psi_{R}^{0}$ as the interpolating field, while

Table 4.2: Zero mode localization for $\psi_{R}^{1}$ interpolating field. The localization of the zero mode and the strength of the coupling to $\psi_{R}^{1}$ are related to the magnitude of the spurion $q$.

\begin{tabular}{ccr}
\hline & Coupling to $\psi_{R}^{1}$ & Zero mode $\left(\psi_{R}=c_{0} \psi_{R}^{0}+c_{1} \psi_{R}^{1}\right)$ \\
\hline$q \ll 1$ & $\approx 1$ & $\psi_{R} \approx \psi_{R}^{1} ;\left(c_{0} \ll 1, c_{1} \approx 1\right)$ \\
$q \gg 1$ & $\ll 1$ & $\psi_{R} \approx \psi_{R}^{0} ;\left(c_{0} \approx 1, c_{1} \ll 1\right)$ \\
\hline
\end{tabular}

maintaining the operator $\mathcal{O}$ coupled to $\psi_{R}^{1}$, in that case the Lagrangian would be

$$
\mathcal{L}_{1}=i \bar{\psi}_{R}^{0} \not \partial\left(1+\frac{1}{q^{2}}\right) \psi_{R}^{0}+\frac{1}{q} \bar{\psi}_{R}^{0} \mathcal{O}+\text { h.c. }
$$

Notice that, even though the coupling was originally placed on site 1 , the technique of integrating out the fields had the side effect of moving the coupling to the site chosen for the interpolating field. The Lagrangian of Eq. (4-10) can be rewritten in a canonical form by taking

$$
\psi_{R}^{0} \rightarrow \frac{1}{\sqrt{1+q^{2}}} \psi_{R}^{0} .
$$

Doing so, gives

$$
\mathcal{L}_{1}=i \bar{\psi}_{R}^{0} \not \partial \psi_{R}^{0}+\frac{1}{\sqrt{1+q^{2}}} \bar{\psi}_{R}^{0} \mathcal{O}+\text { h.c. }
$$

which produces exactly the same physics as the one from Eq. (4-9). This mean that there is a freedom of choice for the interpolating field used in the mechanism, as both choices give the same physics. The same argument can be extended for higher values of $N$. As we previously mention, we can see that the remaining particle $P^{\prime}$, after symmetry breaking, picks up a factor of $q^{2}$, exponentially pushing it towards the boundary of the lattice. This effect is a result of the process of integrating out the fields, that after each equation of motion applied, the Lagrangian picks increasingly powers of the $\mathcal{O}(1)$ parameter $q$. This product of the mechanism is exactly what we want, since it permit us to reach strongly suppressed couplings for sufficiently large 
values of $N$. As our simulations will illustrate, a greater value of $N$ is bound to produce a greater suppression of the interaction when we couple the SM to it. So we can choose the values of $N$ that best fits the correct values for the SM interactions that we wish to reproduce. This way, instead of simply accepting highly unnatural values for the fermion hierarchies, at is in the SM, we only need to accept varying values of $N$ across each generation of fermions. There is where the strength of mechanism lies, as it propose a much more natural approach to the fermion hierarchy problem.

The next step is to promote $m$ and $q$ to $3 \times 3$ matrices $M$ and $Q$ while still working with $N=1$. The corresponding Lagrangian is the following:

$$
\begin{aligned}
\mathcal{L}_{1}=i \bar{\psi}_{L}^{0} \not D \psi_{L}^{0}+i \bar{\psi}_{R}^{0} \not D \psi_{R}^{0}+i \bar{\psi}_{R}^{1} \not D \psi_{R}^{1}-\bar{\psi}_{L}^{0} M \psi_{R}^{0}+\bar{\psi}_{L}^{0} M Q \psi_{R}^{1} \\
-\bar{\psi}_{R}^{0} M^{\dagger} \psi_{L}^{0}+\bar{\psi}_{R}^{1} Q^{\dagger} M^{\dagger} \psi_{L}^{0}
\end{aligned}
$$

From here we proceed in the same manner as before by taking the EulerLagrange equations. We begin with $\psi_{L}^{0}$ :

$$
\not p \psi_{L}^{0}-M\left(\psi_{R}^{0}-Q \psi_{R}^{1}\right)=0 \quad \Longrightarrow \quad \psi_{L}^{0}=\frac{M}{\not p}\left(\psi_{R}^{0}-Q \psi_{R}^{1}\right)
$$

and use this input in the Lagrangian:

$$
\mathcal{L}_{1}=\bar{\psi}_{R}^{0} \not p \psi_{R}^{0}+\bar{\psi}_{R}^{1} \not p \psi_{R}^{1}-\left(\bar{\psi}_{R}^{0}-Q^{\dagger} \bar{\psi}_{R}^{1}\right) \frac{M^{\dagger} M}{\not p}\left(\psi_{R}^{0}-Q \psi_{R}^{1}\right) .
$$

We follow by integrating out $\psi_{R}^{0}$ :

$\not p \psi_{R}^{0}-\frac{M^{\dagger} M}{\not p}\left(\psi_{R}^{0}-Q \psi_{R}^{1}\right)=0 \quad \Longrightarrow \quad \psi_{R}^{0}=\left(M^{\dagger} M-\mathbb{1} p^{2}\right)^{-1} M^{\dagger} M Q \psi_{R}^{1}$

and taking it back to the Lagrangian gives us:

$$
\mathcal{L}_{1}=\bar{\psi}_{R}^{1} \not p\left\{\mathbb{1}+\frac{Q^{\dagger} M^{\dagger} M}{p^{2}}\left[\left(M^{\dagger} M-\mathbb{1} p^{2}\right)^{-1} M^{\dagger} M-\mathbb{1}\right] Q\right\} \psi_{R}^{1} .
$$

We can simplify the expression above by using the identity $(A+B)^{-1} C=$ $\left(C^{-1} A+C^{-1} B\right)^{-1}$, where $A, B$ and $C$ are matrices. This gives us:

$$
\mathcal{L}_{1}=\bar{\psi}_{R}^{1} \not p\left\{\mathbb{1}+\frac{Q^{\dagger} M^{\dagger} M}{p^{2}}\left[\left(\mathbb{1}-\left(M^{\dagger} M\right)^{-1} p^{2}\right)^{-1}-\mathbb{1}\right] Q\right\} \psi_{R}^{1}
$$

Now, in order to be able to take a converging limit when $p^{2} \rightarrow 0$, we need to 
further develop this expression. To achieve this, we can call

$$
\begin{aligned}
Z \equiv \mathbb{1}+\frac{Q^{\dagger} M^{\dagger} M}{p^{2}}\{[\mathbb{1}- & \left.\left.\left(M^{\dagger} M\right)^{-1} p^{2}\right]^{-1}-\mathbb{1}\right\} Q \\
& =\mathbb{1}+Q^{\dagger}\left\{\left[p^{2}\left(M^{\dagger} M\right)^{-1}-\mathbb{1}\right]^{-1}-\frac{M^{\dagger} M}{p^{2}}\right\} Q
\end{aligned}
$$

and this way, our Lagrangian is simply:

$$
\mathcal{L}_{1}=\bar{\psi}_{R}^{1} \not p Z \psi_{R}^{1}
$$

If we multiply by the right by $Q^{-1}\left[\mathbb{1}-\left(M^{\dagger} M\right)^{-1} p^{2}\right]^{-1}$ on the expression for $Z$ we get:

$$
Z Q^{-1}\left[\mathbb{1}-\left(M^{\dagger} M\right)^{-1} p^{2}\right]^{-1}=Q^{-1}\left[\mathbb{1}-\left(M^{\dagger} M\right)^{-1} p^{2}\right]^{-1}+Q^{\dagger}
$$

In this way we got rid of the inconvenient factor of $p^{2}$ on the denominator that was causing the divergence. Now we are ready to take the soft limit $p^{2} \rightarrow 0$ and get:

$$
Z=\mathbb{1}+Q^{\dagger} Q
$$

so that:

$$
\mathcal{L}_{1}=i \bar{\psi}_{R}^{1} \not D\left(\mathbb{1}+Q^{\dagger} Q\right) \psi_{R}^{1}
$$

This is exactly the natural extension of the previous case where we had $m$ and $q$ as scalars. Now we are finally ready to pursuit the most general case for any value of $N$. The Lagrangian for this case is the one below:

$$
\mathcal{L}_{N+1}=\sum_{j=0}^{N+1} i \bar{\psi}_{R}^{j} \not D \psi_{R}^{j}+\sum_{j=0}^{N} i \bar{\psi}_{L}^{j} \not D \psi_{L}^{j}-\sum_{j=0}^{N}\left(\bar{\psi}_{L}^{j} M \psi_{R}^{j}-\bar{\psi}_{L}^{j} M Q \psi_{R}^{j+1}+\text { h.c. }\right) .
$$

The simplest pathway here is to proceed via a proof by induction. For that case one needs to find a relation between $\mathcal{L}_{N+1}$ and $\mathcal{L}_{N}$, this can be easily done by taking a closer look at the Lagrangian. The recurrence relation is the following:

$$
\begin{array}{r}
\mathcal{L}_{N+1}=\mathcal{L}_{N}+\bar{\psi}_{L}^{N} \not p \psi_{L}^{N}+\bar{\psi}_{R}^{N+1} \not p \psi_{R}^{N+1}-\left(\bar{\psi}_{L}^{N} M \psi_{R}^{N}-\bar{\psi}_{L}^{N} M Q \psi_{R}^{N+1}+\bar{\psi}_{R}^{N} M^{\dagger} \psi_{L}^{N}\right. \\
\left.-\bar{\psi}_{R}^{N+1} Q^{\dagger} M^{\dagger} \psi_{L}^{N}\right)+\bar{\psi}_{R}^{N+1} \mathcal{O}, \quad(4-19)
\end{array}
$$

where once again, we have introduced an operator $\mathcal{O}$ as to allow us to have a deeper understanding of the order of magnitude of a coupling with $\psi_{R}^{N+1}$. However, as we saw on the previous examples, when we integrate out the fields of $\mathcal{L}_{N}$, we can write it as $\bar{\psi}_{R}^{N} \not p Z_{N} \psi_{R}^{N}$. Where $Z_{N}$ is a function of $p^{2}, Q$. 
Therefore, we can write:

$$
\begin{aligned}
& \mathcal{L}_{N+1}=\bar{\psi}_{R}^{N} \not p Z_{N} \psi_{R}^{N}+\bar{\psi}_{L}^{N} \not p \psi_{L}^{N}+\bar{\psi}_{R}^{N+1} \not p \psi_{R}^{N+1}-\left(\bar{\psi}_{L}^{N} M \psi_{R}^{N}-\bar{\psi}_{L}^{N} M Q \psi_{R}^{N+1}\right. \\
& \left.+\bar{\psi}_{R}^{N} M^{\dagger} \psi_{L}^{N}-\bar{\psi}_{R}^{N+1} Q^{\dagger} M^{\dagger} \psi_{L}^{N}\right)+\bar{\psi}_{R}^{N+1} \mathcal{O} .
\end{aligned}
$$

Now all we need to do is to use the equations of motion to integrate out the $\psi_{L}^{N}$ and $\psi_{R}^{N}$ fermions, reducing the Lagrangian as something which can be rewriten as $\mathcal{L}_{N+1}=\bar{\psi}_{R}^{N+1} \not p Z_{N+1} \psi_{R}^{N+1}$. This way we will obtain a recurrence relation for $Z_{N}$ under which we can use a proof by induction. We begin by integrating out $\psi_{L}^{N}$ :

$$
\not p \psi_{L}^{N}-M\left(\psi_{R}^{N}-Q \psi_{R}^{N+1}\right)=0 \quad \Longrightarrow \quad \psi_{L}^{N}=\frac{M}{\not p}\left(\psi_{R}^{N}-Q \psi_{R}^{N+1}\right),
$$

then, our Lagrangian will look like:

$$
\mathcal{L}_{N+1}=\bar{\psi}_{R}^{N} \not p Z_{N} \psi_{R}^{N}+\bar{\psi}_{R}^{N+1} \not p \psi_{R}^{N+1}-\left(\bar{\psi}_{R}^{N}-Q \bar{\psi}_{R}^{N+1}\right) \frac{M^{\dagger} M}{\not p}\left(\psi_{R}^{N}-Q \psi_{R}^{N+1}\right)+\bar{\psi}_{R}^{N+1} \mathcal{O}
$$

We proceed by integrating out $\psi_{R}^{N}$ :

$\not p Z_{N} \psi_{R}^{N}-\frac{M^{\dagger} M}{\not p}\left(\psi_{R}^{N}-Q \psi_{R}^{N+1}\right)=0 \Longrightarrow \psi_{R}^{N}=\left(M^{\dagger} M-p^{2} Z_{N}\right)^{-1} M^{\dagger} M Q \psi_{R}^{N+1}$

and, returning it to the Lagrangian, gives us:

$$
\mathcal{L}_{N+1}=\bar{\psi}_{R}^{N} \not p\left\{\mathbb{1}+\frac{Q^{\dagger} M^{\dagger} M}{p^{2}}\left[\left(M^{\dagger} M-p^{2} Z_{N}\right)^{-1} M^{\dagger} M-\mathbb{1}\right] Q\right\} \psi_{R}^{N+1}+\bar{\psi}_{R}^{N+1} \mathcal{O}
$$

So our $Z_{N+1}$ turns out to be the following:

$$
Z_{N+1}=\mathbb{1}+\frac{Q^{\dagger} M^{\dagger} M}{p^{2}}\left[\left(\mathbb{1}-\left(M^{\dagger} M\right)^{-1} p^{2} Z_{N}\right)^{-1}-\mathbb{1}\right] Q
$$

Once again, the factor of $p^{-2}$ is preventing us to do a proper soft limit, so we need to get rid of it by applying $Q^{-1}\left[\mathbb{1}-\left(M^{\dagger} M\right)^{-1} p^{2} Z_{N}\right]^{-1}$ by the right on both sides of the equation:

$$
Z_{N+1} Q^{-1}\left[\mathbb{1}-\left(M^{\dagger} M\right)^{-1} p^{2} Z_{N}\right]=Q^{-1}\left[\mathbb{1}-\left(M^{\dagger} M\right)^{-1} p^{2} Z_{N}\right]+Q^{\dagger} Z_{N} .
$$

Now, if we take $p^{2} \rightarrow 0$ here, we obtain the recurrence relation for $Z_{N}$ :

$$
Z_{N+1}=\mathbb{1}+Q^{\dagger} Z_{N} Q
$$

The previous examples give a hint to how the components of the particle $P^{\prime}$ might look like, leading us in the direction of something polynomial, so we will 
use the following ansatz

$$
Z_{N}=\mathbb{1}+Q^{\dagger} Q+Q^{\dagger^{2}} Q^{2}+\ldots+Q^{\dagger^{N}} Q^{N}
$$

and we will use a proof by induction to show that this is precisely the case. The case where $N=0$ is like we had no clockwork mechanism at all, which trivially means $Z_{0}=\mathbb{1}$. All that is left to do now is to input the proposed expression for $Z_{N}$ into the recurrence relation and check to see if it produces a coherent result:

$$
Z_{N+1}=\mathbb{1}+Q^{\dagger}\left(\mathbb{1}+Q^{\dagger} Q+Q^{\dagger^{2}} Q^{2}+\ldots+Q^{\dagger^{N}} Q^{N}\right) Q
$$

It works perfectly, which means that the tested formula for $Z_{N}$ was right all along. So it is polynomial, in fact:

$$
Z_{N}=\mathbb{1}+Q^{\dagger} Q+Q^{\dagger^{2}} Q^{2}+\ldots+Q^{\dagger^{N}} Q^{N}
$$

In conclusion, the clockwork mechanism produces, after symmetry breaking, a Lagrangian which can be written as a function of only a massless boundary field and polynomial factors of $Q^{\dagger}$ and $Q$. That is:

$$
\mathcal{L}_{N}=i \bar{\psi}_{R}^{N} \not D\left(\sum_{j=0}^{N} Q^{\dagger^{j}} Q^{j}\right) \psi_{R}^{N}+\bar{\psi}_{R}^{N} \mathcal{O},
$$

which, after applying the transformation

$$
\psi_{0}^{N} \rightarrow\left(\sum_{j=0}^{N} Q^{\dagger^{j}} Q^{j}\right)^{-1 / 2} \psi_{0}^{N}
$$

can be written in canonical form as

$$
\mathcal{L}_{N}=i \bar{\psi}_{R}^{N} \not D \psi_{R}^{N}+\bar{\psi}_{R}^{N} Z_{N}^{-1 / 2} \mathcal{O}
$$

where $Z_{N}=\sum_{j=0}^{N} Q^{\dagger^{j}} Q^{j}$.

It is important to point out that we could also have gone through all the same procedures, but instead of choosing to keep the $(N+1)_{\text {th }}$ site, we could have chosen to keep the opposite boundary of the lattice after symmetry breaking. This way, the Lagrangian would look like:

$$
\mathcal{L}_{N}=i \bar{\psi}_{R}^{0} \not D \psi_{R}^{0}+\bar{\psi}_{R}^{0}\left(\sum_{j=0}^{N} Q^{\dagger^{j}} Q^{j}\right)^{-1 / 2} \mathcal{O} .
$$

which does not alter the physics of the system in any way. This means that this mechanism provides a freedom of choice. It does not matter which boundary you pick to represent your physical system after the symmetry breaking, the 
physics of the system will remain unchanged.

It is also worth noticing that this polynomial factor confirms our expectation that, if we couple the SM at the boundary of the lattice via this mechanism, a greater value of $N$ is directly correlated to a greater suppression of the coupling of the $P^{\prime}$ particle to ordinary particles, without the need to introduce small or large parameters. As we will see, this effect comes from the fact that $Z_{N}$ can naturally have eigenvalues $z_{i}$ (where $i=\{1,2,3\}$ and $\left.z_{3}>z_{2}>z_{1}\right)$, such that $z_{3} \gg 1$ and $z_{1} \sim 1$. The former leads to $\mathcal{O}(1)$ couplings (heavy fermions) and the latter to highly suppressed couplings (light fermions). That is the main source of success of the mechanism. This effect allow us to adjust the values of $N$ in our simulation in order to account for the unnatural numbers which have been measured for the Yukawas and mixing angles on the SM and propose a solution for the fermion hierarchy problem.

\section{2}

\section{Hierarchy from product of random matrices}

It has been shown that a property of the product of random matrices of $\mathcal{O}(1)$ is to give out hierarchical eigenvalues. That is, given a matrix of the form

$$
M=M_{1} M_{2} \ldots M_{N}
$$

where each individual matrix multiplied has order-one entries

$$
-1 \leq\left|M_{a b}^{i}\right| \leq 1
$$

will produce eigenvalues $m_{j}$ that obey the following relation

$$
m_{N_{f}} \gg m_{N_{f}-1} \gg \ldots \gg m_{1}
$$

where $N_{f}$ stands for the number of families and is the order of the matrices $M_{i}$. This aspect is explained semi analytically in (15). We will not go into this much detail in here, instead we can ran a simulation of the distribution of the three eigenvalues that convincingly put this topic at rest. The results for $N=2$ and $N=5$ can be seen in Fig. 4.2 and undoubtedly indicate a hierarchical spectra for the eigenvalues.

Going back to our Lagrangian $\mathcal{L}_{N}=i \bar{\psi}_{R}^{N} \not D\left(\sum_{j=0}^{N} Q^{\dagger^{j}} Q^{j}\right) \psi_{R}^{N}$ we can see that it is made of terms $Q^{\dagger^{j}} Q^{j}$ which are products of order-one matrices, which we have just shown that it gives out hierarchical eigenvalues. However, it is still not clear if when we add up each of those terms the resulting sum will also have 
hierarchical eigenvalues. For the special case where the matrix $Q$ is Hermitian it is simple to show that the same result applies to the sum, due to the fact that we can find a base that simultaneously diagonalizes each individual term and the whole sum. In that base, we can write:

$$
\tilde{Z}=U\left(\mathbb{1}+Q^{\dagger} Q+\ldots Q^{\dagger^{N}} Q^{N}\right) U^{\dagger}
$$

that is the same thing as

$$
\tilde{Z}=\left(\begin{array}{ccc}
1 & & \\
& 1 & \\
& & 1
\end{array}\right)+\left(\begin{array}{llll}
q_{1}^{2} & & \\
& q_{2}^{2} & \\
& & q_{3}^{2}
\end{array}\right)+\ldots+\left(\begin{array}{ccc}
q_{1}^{2 N} & & \\
& q_{2}^{2 N} & \\
& & q_{3}^{2 N}
\end{array}\right)
$$

where $q_{i}$ stands for the i-esimal eigenvalue of $Q$. One can run a simulation for the distribution of the eigenvalues of $Q^{\dagger^{j}} Q^{j}$ and check that, as the plot from Fig. 4.3 confirms, for sufficiently large values of $j$ a hierarchical spectrum is reproduced. Thus, one can take $q_{3}^{j} \gg 1, q_{2}^{j} \sim 1$ and $q_{1}^{j} \ll 1$ on Eq. (4-28) and add it all together to get:

$$
\tilde{Z}=\left(\begin{array}{ccc}
z_{1} \sim 1 & & \\
& z_{2}>1 & \\
& & z_{3} \gg 1
\end{array}\right)
$$

where $z_{i}$ are the eigenvalues of $Z$. Now, for the general case where $Q$ is not Hermitian, we again resort to simulations to prove our point. The results for the distribution of the eigenvalues of $Z$, for different values of $N$ are shown in Fig. 4.4 and they undoubtedly show a hierarchical structure between the eigenvalues of $Z$. Now, according to Eq. (4-25), the coupling via the mechanism acquire a factor of $Z^{-1 / 2}$ and, as the distributions of Fig. 4.5 clearly indicates, the hierarchy is preserved when one takes the eigenvalues of $Z^{-1 / 2}$. This means that, if one couples the SM Yukawas to the zero mode of the clockwork mechanism (taking $\mathcal{O}$ as the Yukawas in Eq. (4-25)), one can automatically generate suppressed couplings with eigenvalues that follows a hierarchical spectrum, without the need to introduce unnatural small parameters. This is the main source of success of the mechanism, being able to reproduce the SM fermion mass hierarchy in a natural fashion, as the simulations in the following chapter convincingly demonstrate. 


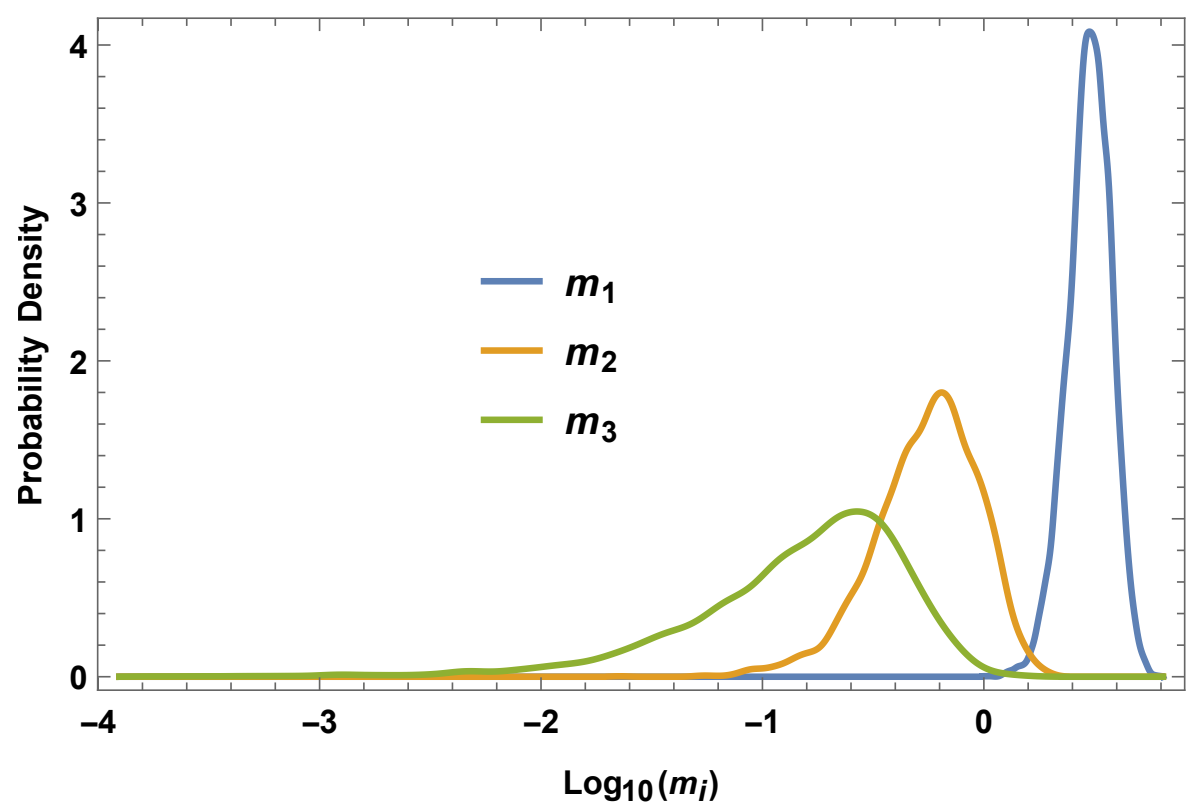

(a) for $\mathrm{N}=2$

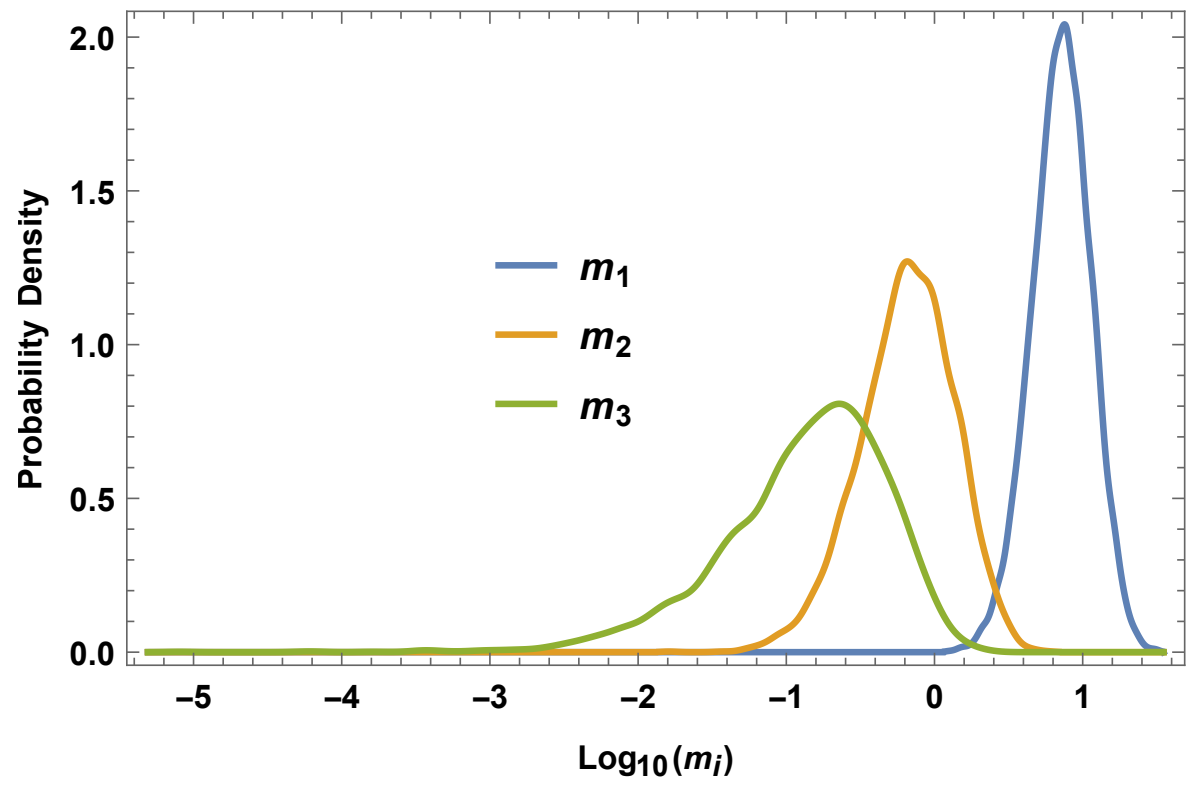

(b) for $\mathrm{N}=5$

Figure 4.2: Distribution of the eigenvalues of product of random matrices. One can undoubtly see a hierarchical distribution for the eigenvalues. 


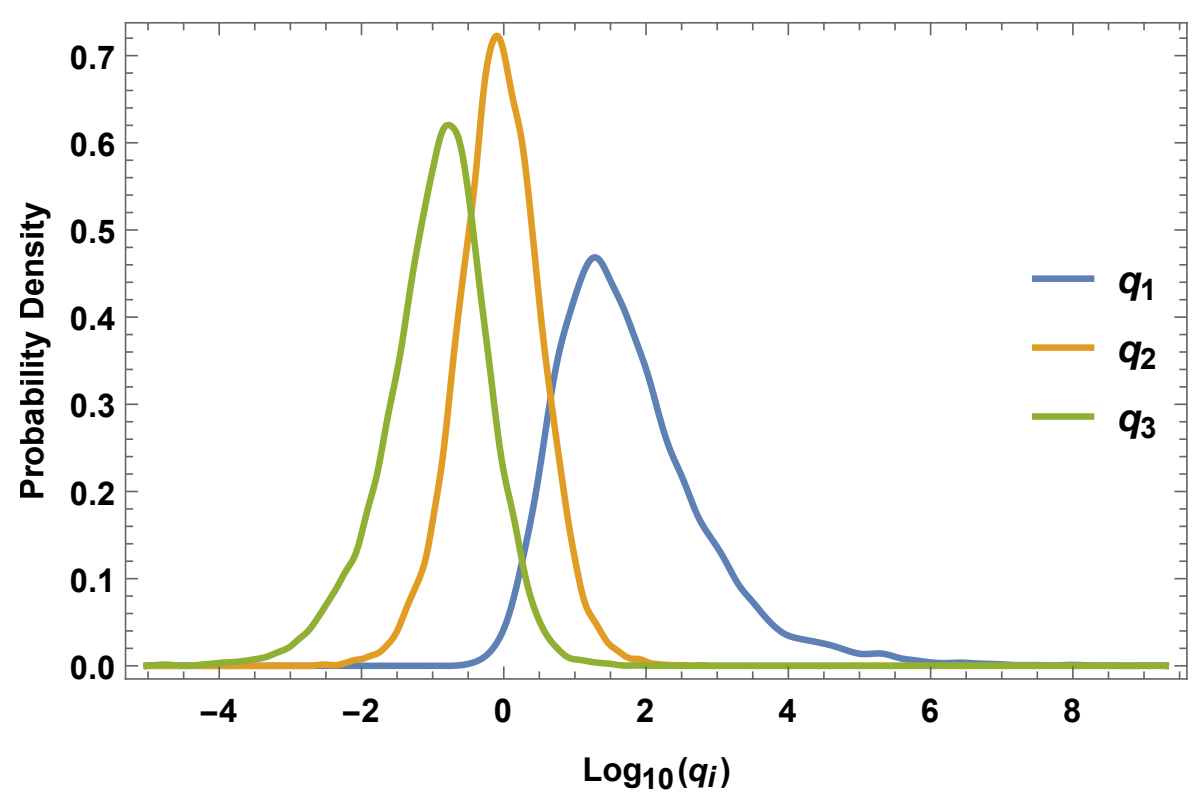

(a) for $\mathrm{N}=2$

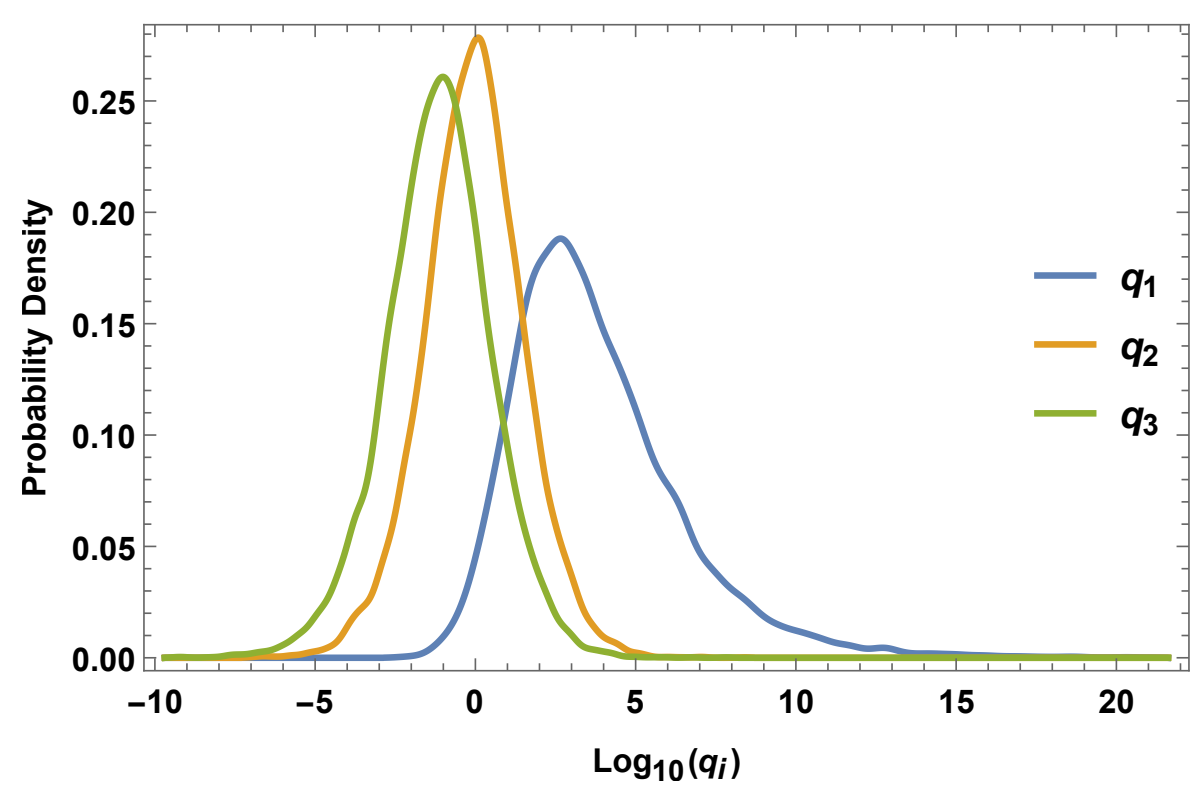

(b) for $\mathrm{N}=5$

Figure 4.3: Distribution of the eigenvalues of $Q^{\dagger^{N}} Q^{N}$. Once again, a hierarchical structure is clearly manifest under the distribution of the eigenvalues. 


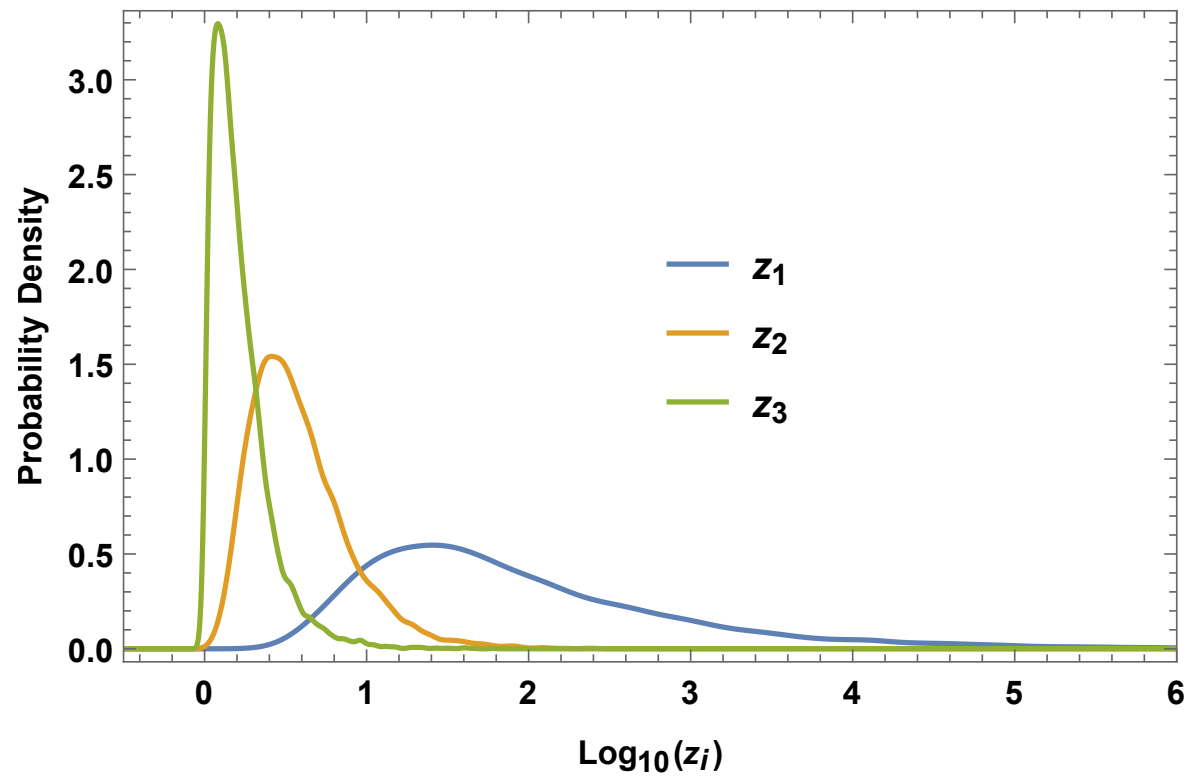

(a) for $\mathrm{N}=2$

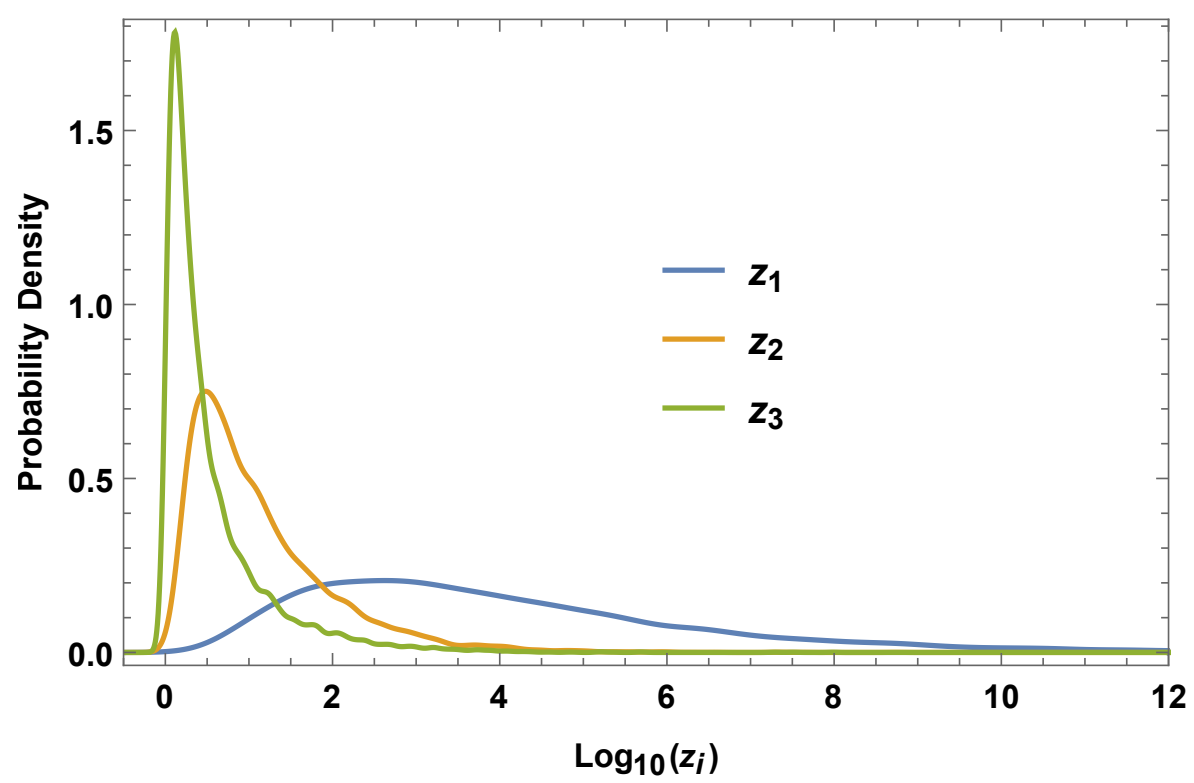

(b) for $\mathrm{N}=5$

Figure 4.4: Distribution of the eigenvalues of $Z$. In this case, the mid eigenvalue seems to be closer to the greater one than to the smaller one. This behaviour agrees with Eq. (4-29). 


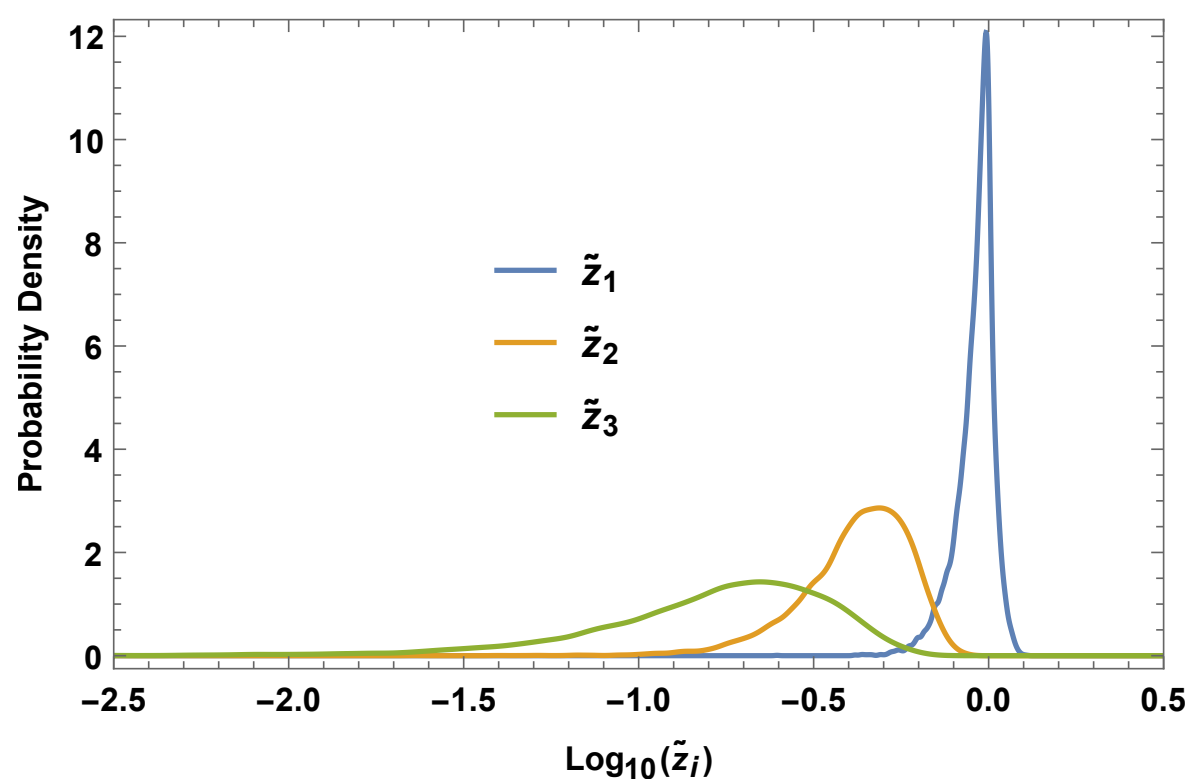

(a) for $\mathrm{N}=2$

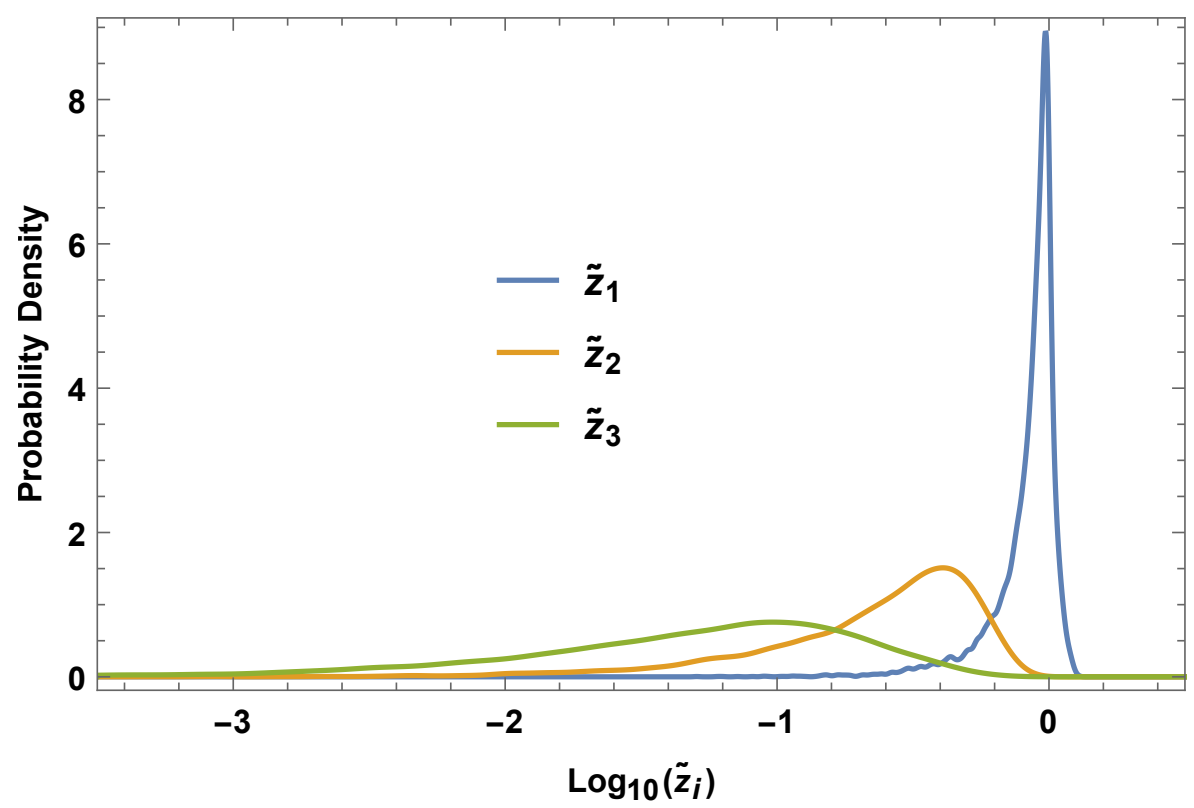

(b) for $\mathrm{N}=5$

Figure 4.5: Distribution of the eigenvalues of $Z^{-1 / 2}$. The hieararchical structure presented here is the responsible for generating the flavour hierarchy in the Clockwork fermion model. 


\section{Simulations}

\section{1}

\section{The clockwork mechanism applied to the Standard Model}

In this chapter we will present the procedures that had been used to perform a simulation for the clockwork mechanism coupled to the SM fermions in attempt to account for the large hierarchy observed in fermion masses, Yukawas and on the CKM and PMNS mixing matrices. To achieve this goal, we need to apply one version of the mechanism for each sector of the SM fermions fields. We begin by introducing new vector-like clockwork particles for each of the SM original fermion fields, like the ones shown in Table 5.1: So we have a total of 5 clockwork mechanism to work with, one for each pair

Table 5.1: Vector-like clockwork fermions introduced. Apart from the neutrinos, there are two chiral fields introduced for each SM field.

\begin{tabular}{cccc}
\hline & Field & Number of particles & Multiplicity under isospin \\
\hline \multirow{4}{*}{ Quarks } & $q_{L}^{i}$ & $N_{q}+1$ & Doublet \\
& $q_{R}^{i}$ & $N_{q}$ & Doublet \\
& $u_{R}^{i}$ & $N_{u}+1$ & Singlet \\
& $u_{L}^{i}$ & $N_{u}$ & Singlet \\
& $d_{R}^{i}$ & $N_{d}+1$ & Singlet \\
& $d_{L}^{i}$ & $N_{d}$ & Singlet \\
\hline \multirow{4}{*}{ Leptons } & $l_{L}^{i}$ & $N_{l}+1$ & Doublet \\
& $l_{R}^{i}$ & $N_{l}$ & Doublet \\
& $e_{R}^{i}$ & $N_{e}+1$ & Singlet \\
& $e_{L}^{i}$ & $N_{e}$ & Singlet \\
\hline
\end{tabular}

of left and right-handed fields. These will play the role of the pairs $\psi_{L}^{i}$ and $\psi_{R}^{i}$ on their own version of the clockwork mechanism. The next step here, as we have seen in the previous chapter, is to break the chiral symmetries for each of those mechanisms until we end up with only five massless particles that picked up a $Z$ factor on their kinetic terms. As it can be seen, we have not considered the mechanism for the neutrinos, as our simulation showed that 
$N_{\nu} \approx 0$ best fit the data for neutrino masses. We did however, consider the existence of a right-handed neutrino with a Majorana mass term $\tilde{M}_{\nu}$ to allow us to compute the PMNS matrix in our simulation. At this point, our full clockwork Lagrangian looks like this:

$$
\begin{aligned}
\mathcal{L}_{\text {quarks }}=i \bar{q}_{L}^{N_{q}} \not D Z_{q} q_{L}^{N_{q}}+i \bar{u}_{R}^{N_{u}} \not D Z_{u} u_{R}^{N_{u}}+i \bar{d}_{R}^{N_{d}} \not D Z_{d} d_{R}^{N_{d}} \\
+\bar{q}_{L}^{N_{q}} Y_{d} H d_{R}^{N_{d}}+\bar{q}_{L}^{N_{q}} Y_{u} \tilde{H} u_{R}^{N_{u}}+\text { h.c. }, \\
\begin{aligned}
\mathcal{L}_{\text {leptons }}=i \bar{l}_{L}^{N_{l}} \not D Z_{l} l_{L}^{N_{l}}+i \bar{e}_{R}^{N_{e}} \not D Z_{e} e_{R}^{N_{e}} & +i \bar{\nu}_{R} \not D \nu_{R}+\bar{l}_{L}^{N_{l}} Y_{e} H e_{R}^{N_{e}} \\
& +\bar{l}_{L}^{N_{l}} Y_{\nu} \tilde{H} \nu_{R}+i\left(\nu_{R}\right)^{c} M_{\nu_{R}} \nu_{R}+\text { h.c. },
\end{aligned}
\end{aligned}
$$

where

$$
Z_{x}=\sum_{j=0}^{N_{x}}\left(Q_{x}^{\dagger}\right)^{j} Q_{x}^{j}
$$

and $x$ can be $q, u, d, l$ or $e$. The Lagrangian is written in terms of the lattice boundaries chosen for each of the five mechanism. However, the way that it is set out, this Lagrangian is not properly canonical, because of the $Z$ factors in the kinetic terms, and cannot be compared to SM Lagrangian as we aim to do. To achieve a canonical shape we perform the following transformations:

$$
\begin{gathered}
q_{L}^{N_{q}} \rightarrow Z_{q}^{-\frac{1}{2}} q_{L} \quad, \quad u_{R}^{N_{u}} \rightarrow Z_{u}^{-\frac{1}{2}} u_{R} \quad, \quad d_{R}^{N_{d}} \rightarrow Z_{d}^{-\frac{1}{2}} d_{R}, \\
l_{L}^{N_{l}} \rightarrow Z_{l}^{-\frac{1}{2}} l_{L} \quad, \quad e_{R}^{N_{e}} \rightarrow Z_{e}^{-\frac{1}{2}} e_{R} .
\end{gathered}
$$

After applying these transformations above, the Lagrangian will be modified by:

$$
\begin{aligned}
\mathcal{L}=\mathcal{L}_{\text {kinetic }} & +\bar{q}_{L} Z_{q}^{-\frac{1}{2}} Y_{d} Z_{d}^{-\frac{1}{2}} H d_{R}+\bar{q}_{L} Z_{q}^{-\frac{1}{2}} Y_{u} Z_{u}^{-\frac{1}{2}} \tilde{H} u_{R} \\
& +\bar{l}_{L} Z_{l}^{-\frac{1}{2}} Y_{e} Z_{e}^{-\frac{1}{2}} H e_{R}+\bar{l}_{L} Z_{l}^{-\frac{1}{2}} Y_{\nu} \tilde{H} \nu_{R}+i\left(\nu_{R}\right)^{c} M_{\nu_{R}} \nu_{R}+\text { h.c. }
\end{aligned}
$$

One can then perform a redefinition of the Yukawas to encompass the factors of $Z$, that is:

$$
\begin{array}{cc}
\tilde{Y}_{d}=Z_{q}^{-\frac{1}{2}} Y_{d} Z_{d}^{-\frac{1}{2}} \quad, & \tilde{Y}_{u}=Z_{q}^{-\frac{1}{2}} Y_{u} Z_{u}^{-\frac{1}{2}}, \\
\tilde{Y}_{e}=Z_{l}^{-\frac{1}{2}} Y_{e} Z_{e}^{-\frac{1}{2}} \quad, & \tilde{Y}_{\nu}=Z_{l}^{-\frac{1}{2}} Y_{\nu} .
\end{array}
$$

As the results of our simulation prove, these factors of $Z$ in our model are precisely the ones responsible for introducing the hierarchy in the Yukawas. Our Lagrangian then becomes simply: 
$\mathcal{L}=\mathcal{L}_{\text {kinetic }}+\bar{q}_{L} \tilde{Y}_{d} H d_{R}+\bar{q}_{L} \tilde{Y}_{u} \tilde{H} u_{R}+\bar{l}_{L} \tilde{Y}_{e} H e_{R}+\bar{l}_{L} \tilde{Y}_{\nu} \tilde{H} \nu_{R}+i\left(\nu_{R}\right)^{c} M_{\nu_{R}} \nu_{R}+$ h.c..

To obtain the fermion masses we need to diagonalize our new Yukawas, as it is also done in the SM. Once again, this is done by introducing unitary matrices $U$ such that:

$$
\begin{array}{cc}
\frac{v^{2}}{2} \tilde{Y}_{d} \tilde{Y}_{d}^{\dagger}=U_{d} M_{d}^{2} U_{d}^{\dagger} & \frac{v^{2}}{2} \tilde{Y}_{u} \tilde{Y}_{u}^{\dagger}=U_{u} M_{u}^{2} U_{u}^{\dagger}, \\
\frac{v^{2}}{2} \tilde{Y}_{e} \tilde{Y}_{e}^{\dagger}=U_{e} M_{e}^{2} U_{e}^{\dagger} & , \quad \tilde{M}_{\nu_{L}}=U_{\nu} M_{\nu_{L}} U_{\nu}^{T},
\end{array}
$$

where the matrices $M$ are all diagonal and

$$
M_{\nu_{L}}=\frac{v^{2}}{2} Y_{\nu} M_{\nu_{R}}^{-1} Y_{\nu}^{T}
$$

Then, the eigenvalues of $\tilde{Y}_{u} \tilde{Y}_{u}^{\dagger}, \tilde{Y}_{d} \tilde{Y}_{d}^{\dagger}$ and $\tilde{Y}_{e} \tilde{Y}_{e}^{\dagger}$ give the squared masses of the SM fermions of the up sector, down sector and charged leptons, respectively. These can be obtained numerically within the simulations. Whereas, the mass mixing matrices are given by:

$$
\begin{gathered}
V_{C K M}=U_{u}^{\dagger} U_{d}, \\
V_{P M N S}=U_{\nu}^{\dagger} U_{e} .
\end{gathered}
$$

Again, we can obtain these numerically by calculating the eigenvectors of $\tilde{Y}_{u} \tilde{Y}_{u}^{\dagger}$, $\tilde{Y}_{d} \tilde{Y}_{d}^{\dagger}, \tilde{Y}_{e} \tilde{Y}_{e}^{\dagger}$ and $\tilde{M}_{\nu} \tilde{M}_{\nu}^{\dagger}$. It is interesting to point out that the physical Yukawas acquire, via this mechanism, a structure that resembles the Froggatt Nielsen model. To illustrate this point, one can take a look at the up Yukawa, for example:

$$
\tilde{Y}_{u}=Z_{q}^{-\frac{1}{2}} Y_{d} Z_{d}^{-\frac{1}{2}}=\left(U_{q} \tilde{Z}_{q}^{-\frac{1}{2}} U_{q}^{\dagger}\right) Y_{d}\left(U_{d} \tilde{Z}_{d}^{-\frac{1}{2}} U_{d}^{\dagger}\right)
$$

where $\tilde{Z}$ is diagonal and $U$ are $\mathcal{O}(1)$ matrices. Now writing it explicitly in terms of the eigenvalues $\epsilon$, we get:

$$
\tilde{Y}_{u}=U_{q}\left(\begin{array}{ccc}
\epsilon_{1}^{q} & & \\
& \epsilon_{2}^{q} & \\
& & \epsilon_{1}^{q}
\end{array}\right) U_{q}^{\dagger} Y_{u} U_{u}\left(\begin{array}{ccc}
\epsilon_{1}^{u} & & \\
& \epsilon_{2}^{u} & \\
& & \epsilon_{1}^{u}
\end{array}\right) U_{u}^{\dagger} .
$$

Now, since we have shown in the previous chapter that the matrices $Z$ have hierarchical eigenvalues and the presence of $\mathcal{O}(1)$ matrices does not change that fact, this overall structure is very similar to the one we had in the Froggatt Nielsen mechanism:

$$
M_{u}=\left(\begin{array}{ccc}
\epsilon^{4} & & \\
& \epsilon^{2} & \\
& & 1
\end{array}\right) Y_{u}\left(\begin{array}{lll}
\epsilon^{4} & & \\
& \epsilon^{2} & \\
& & 1
\end{array}\right) .
$$


This is another clear indication why the mechanism works at dealing with the fermion hierarchy problem.

\section{2}

\section{Computational analysis}

\subsection{1}

\section{Assumptions and procedures}

The goal of our simulation is to find the number of clockwork fermions $N$ that best reproduce the values and hierarchical structure for the fermion masses and entries on the mass mixing matrices $V_{C K M}$ and $V_{P M N S}$ which have been found experimentally. Since we want our model to be more natural, we will not make any assumption whether the ten matrices (two for each mechanism) $M$ and $K=M Q$ are small or large. Instead, we will simply take them to be random $3 \times 3$ complex matrices of $\mathcal{O}(1)$ with entries that uniformly span from $-1-i$ to $1+i$, similar to what have been done in Chapter 2 . In other words:

$$
\begin{array}{ll}
0 \leq\left|(M)_{i j}\right| \leq 1, & \text { for } i, j=1,2,3, \\
0 \leq\left|(K)_{i j}\right| \leq 1, & \text { for } i, j=1,2,3 .
\end{array}
$$

The clockwork mechanism works very well when recreating hierarchies between masses of particles in the same sector but across different generations. However, when it comes to replicating an overall hierarchy between distinct sectors, the mechanism struggles to find a fit to data. For example, as we have seen,

$$
\frac{y_{t}}{y_{b}} \sim 40
$$

So, between the up and down sector there is a clear hierarchy, which the mechanism cannot consistently reproduce. In order to go around this obstacle, we introduce free parameters $c_{i}$ 's (where $i=u, d, e$ ) which are numbers that multiply the Yukawa interactions terms in the Lagrangian. These parameters can properly account for the different orders of magnitude of masses across all sectors, fixing the flaw on the mechanism. So the fixed Lagrangian looks like this:

$$
\begin{aligned}
\mathcal{L}=\mathcal{L}_{\text {kinetic }}+c_{d} \bar{q}_{L} \tilde{Y}_{d} H d_{R}+c_{u} \bar{q}_{L} \tilde{Y}_{u} \tilde{H} u_{R}+c_{e} \bar{l}_{L} \tilde{Y}_{e} H e_{R} \\
+\bar{l}_{L} \tilde{Y}_{\nu} \tilde{H} \nu_{R}+i\left(\nu_{R}\right)^{c} \tilde{M}_{\nu_{R}} \nu_{R}+\text { h.c.. }
\end{aligned}
$$

In this work we are making the argument to use the clockwork mechanism for the purpose of building a more natural theory for fermions, so it may seen 
counterintuitive to force these free parameters $c_{i}$ 's into the theory. One can give two possible explanations for the origin of these parameters. It can be inspired by supersymmetry (SUSY) (70) or the two Higgs doublet model (2HDM) (71) which predicts the existence of multiple Higgs particles, and so $H$ and $\tilde{H}$ no longer represent the same particle, but instead each one are now related to different Higgs particle with different VEVs. That is:

$$
H=H_{1} \quad, \quad \tilde{H}=H_{2}
$$

The model can be parameterized such that:

$$
\tan \beta=\frac{\left\langle H_{1}\right\rangle}{\left\langle H_{2}\right\rangle}, \quad\left\{\begin{array}{l}
\left\langle H_{1}\right\rangle=v \cos \beta \\
\left\langle H_{2}\right\rangle=v \sin \beta
\end{array},\right.
$$

where $\tan \beta$ is large $(\tan \beta \sim 50)$, so we can make the approximations:

$$
c_{u} \sim \sin \beta \approx 1 \quad, \quad c_{d} \sim c_{e} \sim \cos \beta \sim \frac{1}{\tan \beta} \approx \frac{1}{50} .
$$

Another possible explanation can be simply that, for some reason, the clockwork spurions ( $M$ and $K$ ) for the down and lepton sectors have their symmetries broken at a much higher energy scale than the ones from the up sector, causing them to acquire greater VEVs. That is:

$$
\left\langle M_{d}\right\rangle \sim\left\langle M_{e}\right\rangle \gg\left\langle M_{u}\right\rangle
$$

This effect can be translated as if there was a shift in the order of the matrices $Q=M^{-1} K$ in the down and lepton sectors, such that

$$
\mathcal{O}\left(Q_{d}\right) \sim \mathcal{O}\left(Q_{e}\right)>1
$$

In order to find the optimal results for our free parameters we made use of the $\chi^{2}$ test, which gives an indication to how likely it is that a random value, taken from the distribution of the simulation, matches the experimental data. Minimizing the value for $\chi^{2}$ gives us a convenient way to manipulate our free parameters until we find the best fit for the data observed experimentally. The $\chi^{2}$ is computed as:

$$
\chi^{2}\left(c_{i}\right)=\sum_{i, j=1}^{N_{\text {dof }}} \frac{\left(x_{i}^{e x p}-c_{i}\left\langle x_{i}\right\rangle\right)}{\sigma_{x_{i}}} C_{i j} \frac{\left(x_{j}^{e x p}-c_{j}\left\langle x_{j}\right\rangle\right)}{\sigma_{x_{j}}},
$$

where $x_{i}^{\exp }$ is the experimental value for the physical quantity at hand, $x_{i}$ is the corresponding simulated distribution, $\sigma_{x_{i}}$ is the standard deviation of 
such distribution, $C_{i j}$ is the correlation matrix between $x_{i}$ and $x_{j}, N_{d o f}$ is the number of degrees of freedom, which here is taken as the number of observables considered for the analysis, and $c_{i}$ is the parameter introduced to account for the hierarchy between different sectors. Then the parameters $c_{i}$ are determined by solving the system of equations

$$
\frac{\partial}{\partial c_{i}} \chi^{2}\left(c_{i}\right)=0
$$

which guarantees the minimum value for $\chi^{2}$, by taking

$$
\chi_{\min }^{2}=\chi^{2}\left(c_{i}^{\text {opt }}\right),
$$

where $c_{i}^{\text {opt }}$ are the solutions for Eq. (5-23). The test was performed separately for the quark and lepton sectors, since there is no interference between the mechanisms applied for each of those distinct sectors.

\section{2 .2}

\section{Results for the quark sector}

We begin our statistical analysis by taking a look at the quark sector. The relevant variables considered as inputs for the $\chi^{2}$ on the quark sector and it's experimental values are presented in the Table 5.2, while the parameters that best fit the data for the quark sector are presented in Table 5.3. The results for the quark sector has shown to be satisfying, since, as it is clear from the plots in Fig. 5.1 and Fig. 5.2, the experimental values overall fitted well within the range of the distributions. This proves the power of the clockwork mechanism for resolving the hierarchy problem, as it can reproduce the experimental data in a much more natural sense. The results for $c_{u} \sim 1$ and $c_{d} \sim \frac{1}{50}$ are within expectations. The spotlight in the quark sector goes to the distribution for $y_{t}$, which, when other model struggles to reconcile its large value $\left(y_{t} \sim \mathcal{O}(1)\right)$ and often requires the use of assumptions such as $K \ll M$, turning all couplings suppressed including the top, or the use of the mechanism in the quark sector exclusively to the first two generations (as seen in (72)), this version of the clockwork mechanism has proven that it can match beautifully with the experimental value without the need to introduce any additional assumption and, therefore, in this manner can be regarded as more successful. It is also important to highlight the satisfactory result for the distribution of $y_{b}$, which is notably harder to reproduce in other flavour models. 
Table 5.2: Quark sector variables and their experimental values (7). Once again, the experimental uncertainties were not taken into account as their contribution are neglectable.

\begin{tabular}{cc}
\hline Quantity & Experimental value \\
\hline$y_{d}$ & $1.364 \times 10^{-5}$ \\
$y_{s}$ & $2.70 \times 10^{-4}$ \\
$y_{b}$ & $1.388 \times 10^{-2}$ \\
\hline$y_{u}$ & $6.3 \times 10^{-6}$ \\
$y_{c}$ & $3.104 \times 10^{-3}$ \\
$y_{t}$ & 0.8685 \\
\hline $\sin \theta_{12}$ & 0.225 \\
$\sin \theta_{23}$ & 0.042 \\
$\sin \theta_{13}$ & $3.55 \times 10^{-3}$ \\
$J$ & $3.18 \times 10^{-5}$ \\
\hline
\end{tabular}

Table 5.3: Quark sector best fit free parameters obtained through simulations. The values for $c_{u}$ and $c_{d}$ agrees with the prediction from Eq. (5-21), while the small value for $\chi_{\min }^{2}$ indicates that the distributions obtained match the experimental data. The 10 degrees are freedom involved in the computation of $\chi_{\min }^{2}$ are the ones related to the parameters presented in Table 5.2.

\begin{tabular}{cc}
\hline Quantity & Value \\
\hline$N_{q}$ & 9 \\
$N_{u}$ & 9 \\
$N_{d}$ & 6 \\
\hline$c_{u}$ & 1.70 \\
$c_{d}$ & 0.03 \\
\hline$\chi_{\min }^{2}$ & 8.59 \\
\hline
\end{tabular}




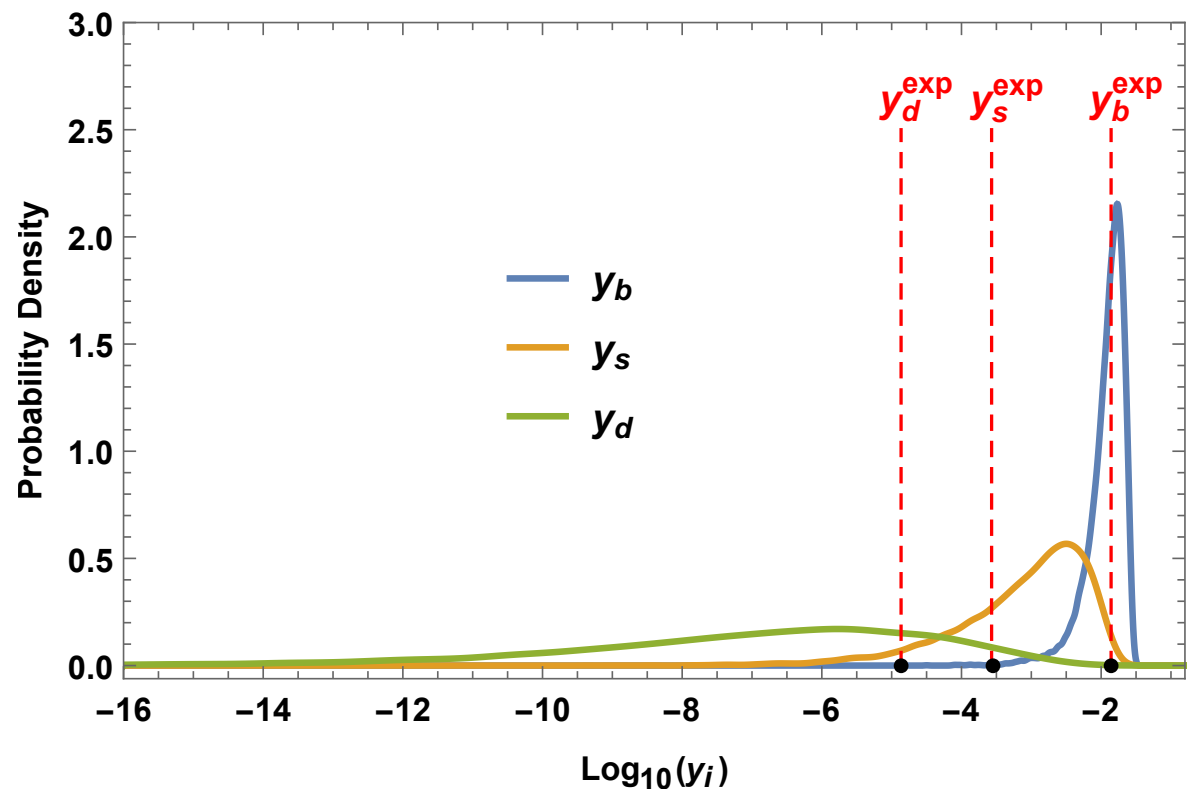

(a) Down sector masses

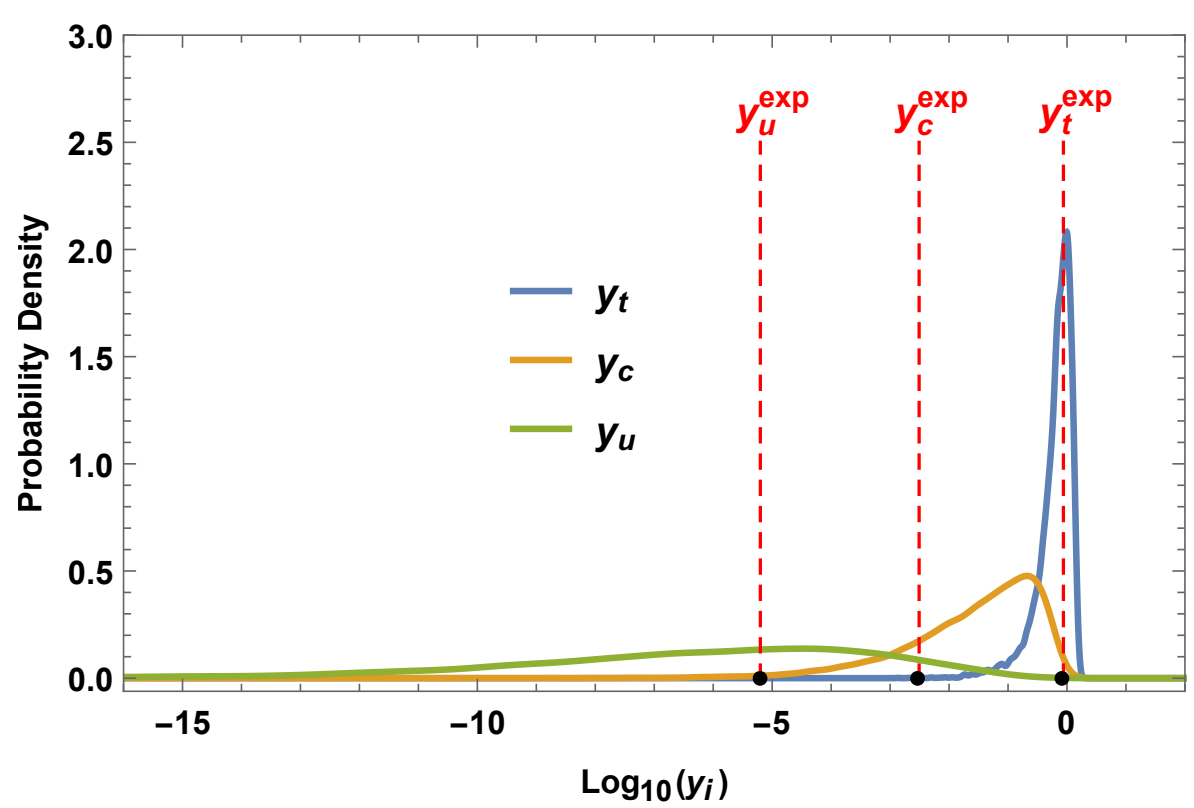

(b) Up sector masses

Figure 5.1: Quark masses distributions for $N_{q}=9, N_{u}=9, N_{d}=6, c_{u}=1.70$ and $c_{d}=0.03$. The experimental values fall well within the reach of the distributions, especially for $y_{t}$ and $y_{b}$. 


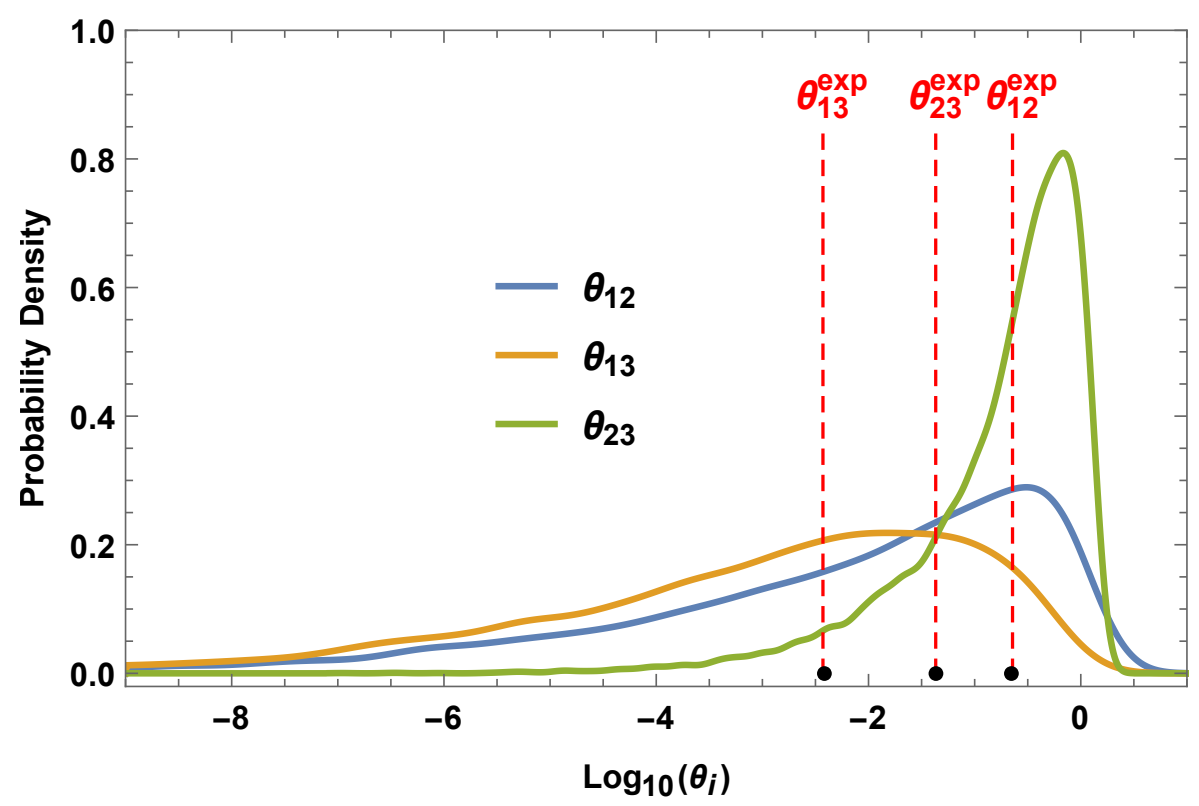

(a) CKM mixing angles

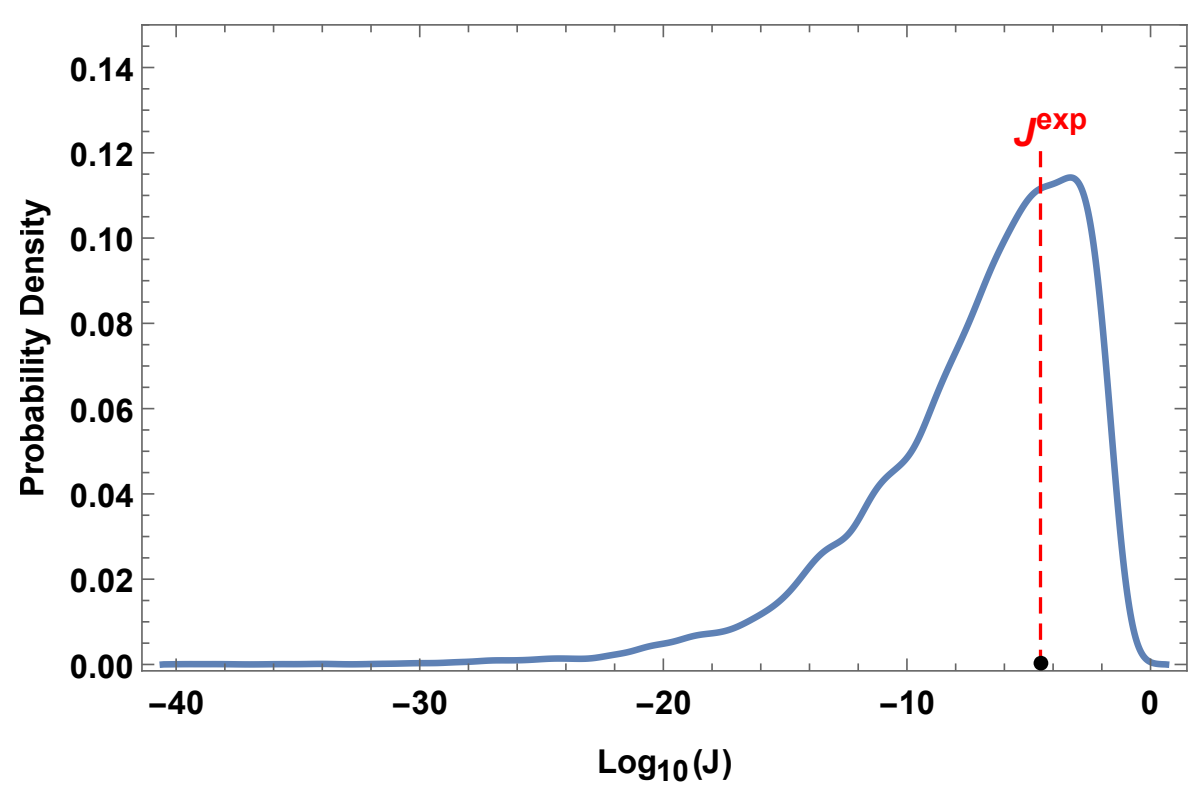

(b) Jarlskog invariant

Figure 5.2: Quark mixing distributions for $N_{q}=9, N_{u}=9, N_{d}=6, c_{u}=1.70$ and $c_{d}=0.03$. The experimentally measured values fit greatly with the distributions obtained. 


\section{2 .3}

\section{Results for the lepton sector}

The experimental data for the lepton sector are presented in Table 5.4 and the parameters that best fit the data for the lepton sector are presented in Table 5.5. Here we have considered the normal ordering in the neutrino sector (that is, $\Delta m_{31}^{2}>0$ ) and $\theta_{23}$ in the first octant (that is, $\theta_{23}<\frac{\pi}{4}$ ). Once again, as the plots from Fig. 5.3 and Fig. 5.4 show, the results in the lepton sector were deemed rather pleasing. According to the plots, one can safely say that the distributions for the considered variables is likely to randomly fall in range of the experimental data. In other words, the quantities measured experimentally matches suitably with the simulations. In addition, the results for $c_{e} \sim \frac{1}{50}$ and $m_{R} \sim 10^{14} \mathrm{GeV}$ fits very well with the predictions.

Table 5.4: Lepton sector variables and their experimental values (7). Once again, the experimental uncertainties were not taken into account as their contribution are neglectable.

\begin{tabular}{cc}
\hline Quantity & Experimental value \\
\hline$y_{e}$ & $2.8482 \times 10^{-6}$ \\
$y_{\mu}$ & $6.0127 \times 10^{-4}$ \\
$y_{\tau}$ & $1.02213 \times 10^{-2}$ \\
\hline$\Delta m_{12}^{2}$ & $7.53 \times 10^{-5} \mathrm{eV}^{2}$ \\
$\Delta m_{23}^{2}$ & $2.51 \times 10^{-3} \mathrm{eV}^{2}$ \\
\hline $\sin ^{2}\left(\theta_{12}\right)$ & 0.307 \\
$\sin ^{2}\left(\theta_{23}\right)$ & 0.417 \\
$\sin ^{2}\left(\theta_{13}\right)$ & $2.12 \times 10^{-2}$ \\
$J_{C P}$ & -0.027 \\
\hline
\end{tabular}


Table 5.5: Lepton sector best fit free parameters obtained through simulations. The values for $c_{e}$ agrees with the prediction from Eq. (5-21), while the small value for $\chi_{\min }^{2}$ indicates that the distributions obtained match the experimental data. The 9 degrees are freedom involved in the computation of $\chi_{\min }^{2}$ are the ones related to the parameters presented in Table 5.2.

\begin{tabular}{cc}
\hline Quantity & Value \\
\hline$N_{l}$ & 2 \\
$N_{e}$ & 9 \\
$N_{\nu}$ & 0 \\
\hline$c_{e}$ & 0.02 \\
$m_{R}$ & $4.26 \times 10^{14} \mathrm{GeV}$ \\
\hline$\chi_{\min }^{2}$ & 1.86 \\
\hline
\end{tabular}




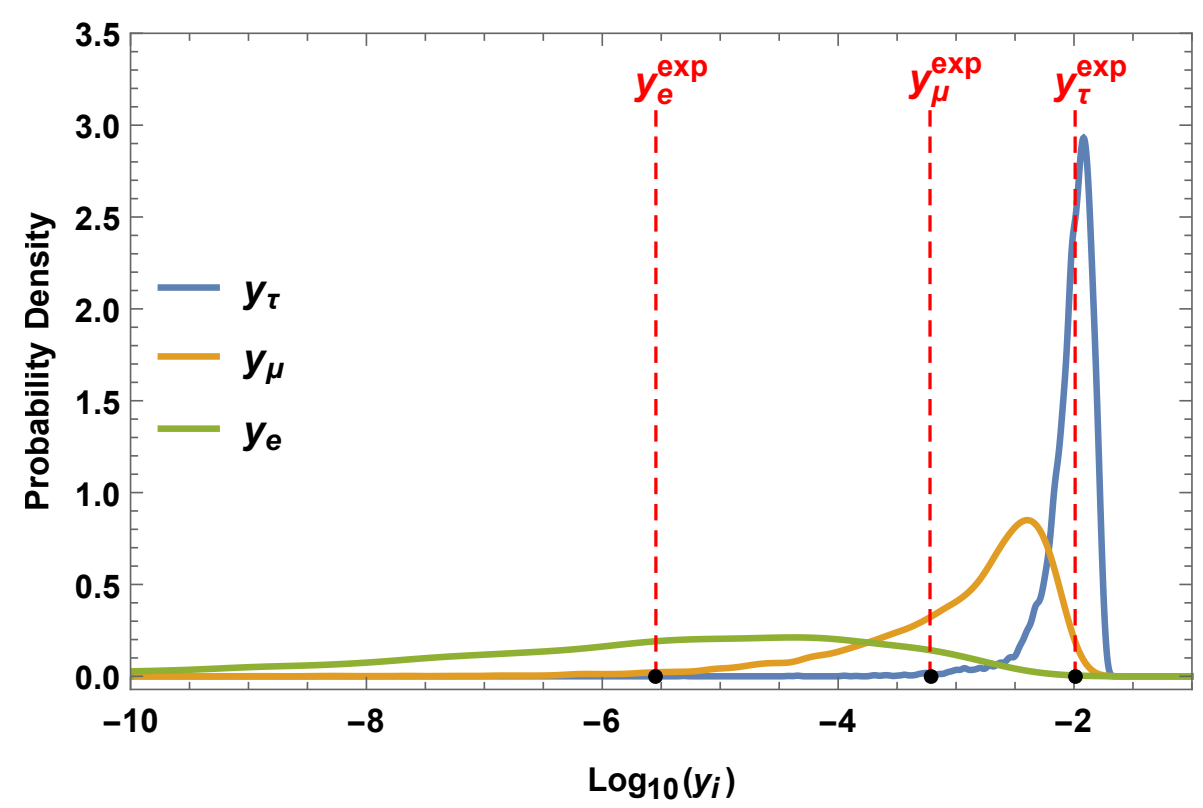

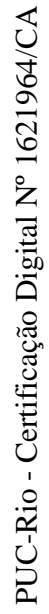

(a) Charged leptons sector masses

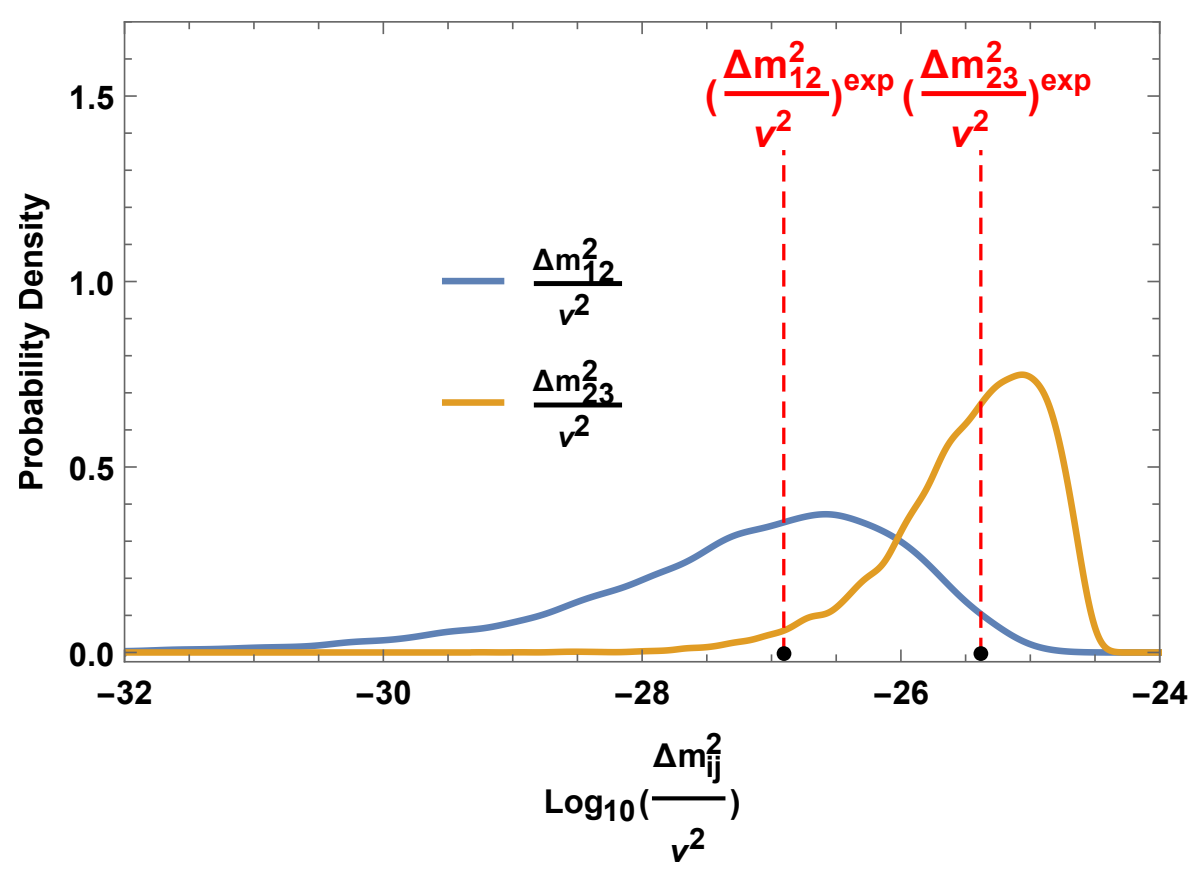

(b) Neutrino squared masses

Figure 5.3: Lepton masses distributions for $N_{l}=2, N_{e}=9, N_{\nu}=0, c_{d}=0.02$ and $m_{R}=4.26 \times 10^{14} \mathrm{GeV}$. The experimental values fall well within the reach of the distributions. 


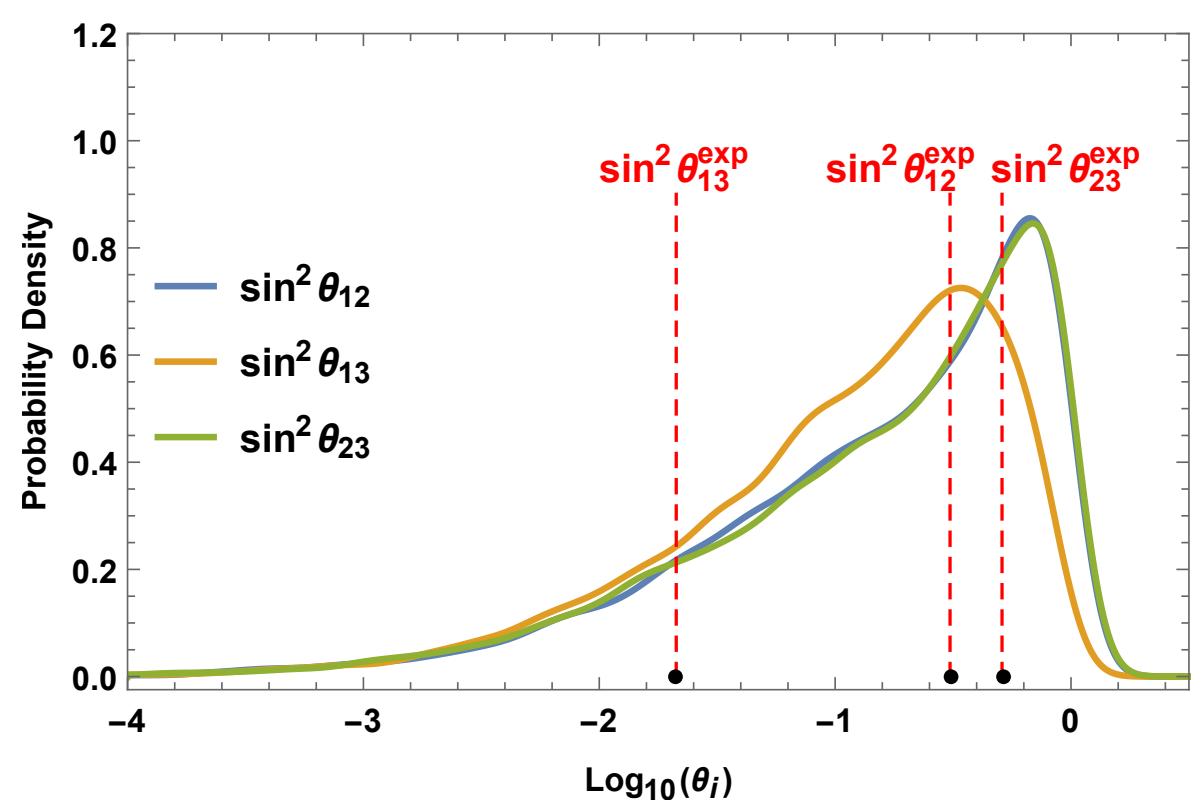

(a) PMNS mixing angles

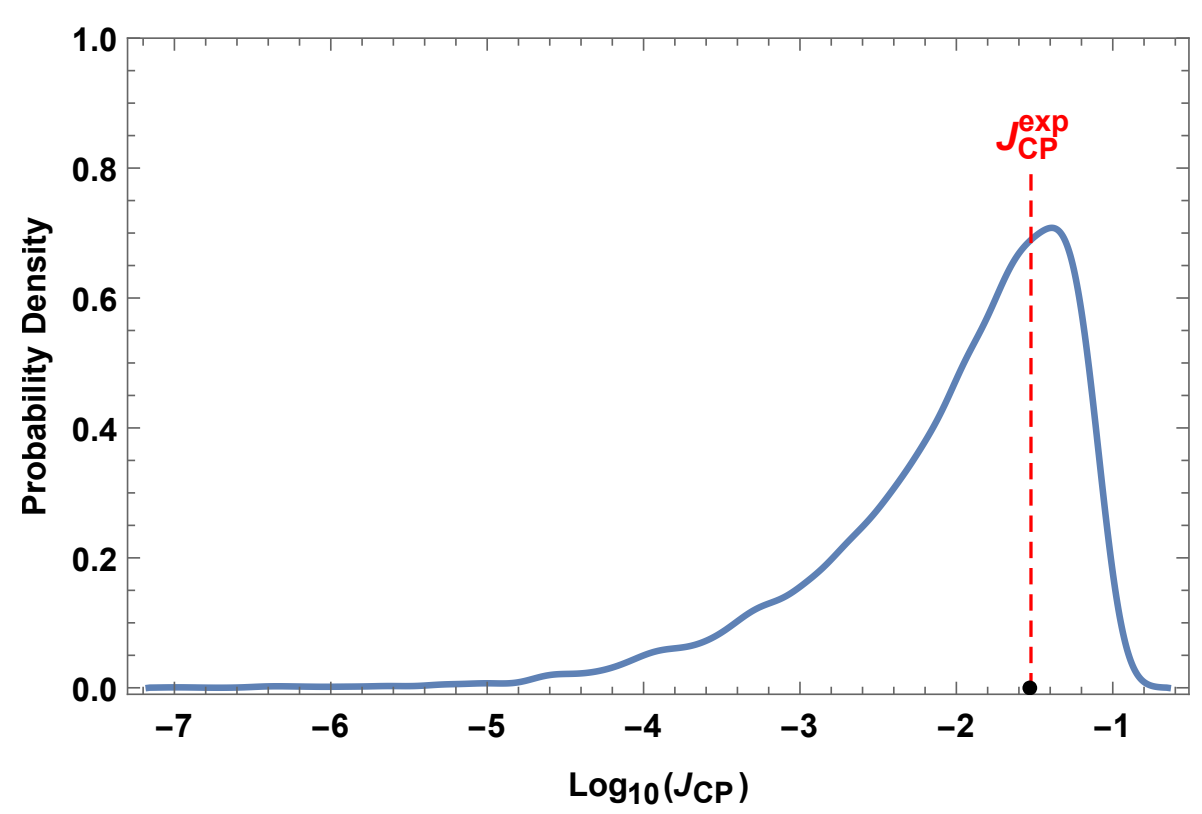

(b) Rephasing invariant

Figure 5.4: Lepton mixing distributions for $N_{l}=2, N_{e}=9, N_{\nu}=0, c_{d}=0.02$ and $m_{R}=4.26 \times 10^{14} \mathrm{GeV}$. The experimentally measured values fit greatly with the distributions obtained. 


\section{6}

\section{Flavour changing neutral currents}

\section{1}

\section{Building an effective field theory}

In this chapter we will probe the existence of Flavour Changing Neutral Currents (FCNC) in the model at hand. The only origins of flavour mixing in the SM are the CKM matrix, in the quark sector, and PMNS matrix, in the lepton sector. The CKM matrix is only present in the $\mathrm{W}$ sector, being so, since the $\mathrm{W}$ boson is charged, all processes involving flavour changing at tree level must be mediated by a charged current. Whereas, in the lepton sector, in addition to the neutrinos oscillations, charged leptons flavor changing processes are suppressed by the difference in neutrino masses. To see why this is the case, suppose if there was no flavour changing in the charged lepton sector, this would mean the existence of a global charged leptonic family number symmetry

$$
U(1)_{e} \times U(1)_{\mu} \times U(1)_{\tau}
$$

in the SM. Under such symmetry, the charged lepton fields $\{e, \mu, \tau\}$ would have the following charges: $\{(1,0,0) ;(0,1,0) ;(0,0,1)\}$. Now, to keep terms in the Lagrangian like

$$
\Delta \mathcal{L}=\bar{e} W \nu_{e}, \quad \Delta \mathcal{L}=\bar{\mu} \mathscr{W} \nu_{\mu}, \quad \Delta \mathcal{L}=\bar{\tau} W \nu_{\tau}
$$

invariant under this symmetry, the neutrino fields $\left\{\nu_{e}, \nu_{\mu}, \nu_{\tau}\right\}$ would need charges $\{(1,0,0) ;(0,1,0) ;(0,0,1)\}$ as well. However, since it is known that neutrino mixing exists, the only way that one could maintain the off-diagonal neutrino mass terms in

$$
M=\left(\begin{array}{lll}
\bar{\nu}_{e} & \bar{\nu}_{\mu} & \bar{\nu}_{\tau}
\end{array}\right) U^{\dagger}\left(\begin{array}{ccc}
m_{1} & & \\
& m_{2} & \\
& & m_{3}
\end{array}\right) U\left(\begin{array}{c}
\nu_{e} \\
\nu_{\mu} \\
\nu_{\tau}
\end{array}\right)
$$

invariant under $U(1)_{e} \times U(1)_{\mu} \times U(1)_{\tau}$ was if we had $m_{1}=m_{2}=m_{3}$. That is why any flavour changing process in the lepton sector must be proportional 
to some $\left|\Delta m_{i j}^{\nu}\right|=\left|m_{i}^{\nu}-m_{j}^{\nu}\right|$, where $i \neq j=\{1,2,3\}$, and since the neutrinos masses are known to be very small $(\sim 0.1 \mathrm{eV})$, those processes are bound to be heavily suppressed. That is the main reason why the discovery of FCNC's is important for BSM physics (73). That have been many experiments searching for these processes (74), (75), however, up to the present date, none has emerged successful at detecting them.

The appearance of FCNC is achievable by expanding the Clockwork Fermion Lagrangian into an Effective Field Theory (EFT) to account for dimension- 6 operators. To illustrate the point, let us first take a look at a simplified model $\left(N_{l}=0, N_{e}=1\right)$ and later generalize it for any pair of $N_{l}, N_{e}$. The corresponding Lagrangian is as follows:

$\mathcal{L}=i \bar{l}_{L} \not D l_{L}+i \bar{e}_{L}^{0} \not D e_{L}^{0}+i \bar{e}_{R}^{0} \not D e_{R}^{0}+i \bar{e}_{R}^{1} \not D e_{R}^{1}-\bar{e}_{L}^{0} M e_{R}^{0}+\bar{e}_{L}^{0} M Q e_{R}^{1}+\bar{l}_{L} H Y_{e} e_{R}^{1}+$ h.c. .

After integrating out the $e_{L}^{0}$ and $e_{R}^{0}$ fields, we get

$$
Z=Q^{\dagger}\left[\not D^{2}\left(M^{\dagger} M\right)^{-1}+\mathbb{1}\right]^{-1} Q+\mathbb{1}
$$

which can be Taylor expanded into

$$
Z=Q^{\dagger}\left[\mathbb{1}-\not D^{2}\left(M^{\dagger} M\right)^{-1}+\mathcal{O}\left(\not D^{4}\right)\right] Q+\mathbb{1}
$$

and therefore, the complete Lagrangian can be rewritten as:

$\mathcal{L}=i \bar{l}_{L} \not D l_{L}+i \bar{e}_{R} \not D e_{R}+i \bar{e}_{R} \not D Q^{\dagger}\left[\mathbb{1}-\not D^{2}\left(M^{\dagger} M\right)^{-1}\right] Q e_{R}+\bar{l}_{L} H Y_{e} e_{R}+\bar{e}_{R} Y_{e}^{\dagger} H^{\dagger} l_{L}$,

which, by opening up the brackets, gives us, in a addition to the dimension- 4 Lagrangian which we have seen on Chap. 4, a dimension-6 operator:

$$
\mathcal{L}_{4}=i \bar{l}_{L} \not D l_{L}+i \bar{e}_{R}\left(\mathbb{1}+Q^{\dagger} Q\right) \not D e_{R}+\bar{l}_{L} H Y_{e} e_{R}+\bar{e}_{R} Y_{e}^{\dagger} H^{\dagger} l_{L}
$$

$$
\mathcal{L}_{6}=-i \bar{e}_{R} Q^{\dagger}\left(M^{\dagger} M\right)^{-1} Q \not D^{3} e_{R}
$$

The FCNC processes are already present in this dimension-6 Lagrangian, but in this basis the Feynman Rules are more complicated, so to simplify the calculations, one can use a trick which involves taking the equations of motion of dimension-4 Lagrangian and apply then at the dimension-6 Lagrangian. This procedure leaves the complete Lagrangian unaltered up to order of dimension6. Let's see why this is the case. Suppose we take a general Lagrangian involving some field $\phi$ with dimension-4 operators and a suppressed dimension6 operator, so we have

$$
\mathcal{L}=\mathcal{L}_{4}(\phi)+\epsilon \mathcal{L}_{6}(\phi),
$$

where $\epsilon$ is some small parameter. Now we can make a redefinition of the field by taking 


$$
\phi=\phi^{\prime}+\epsilon F\left(\phi^{\prime}\right)
$$

where $F\left(\phi^{\prime}\right)$ is some functional of $\phi^{\prime}$, and express $\mathcal{L}$ in terms of the new field:

$\mathcal{L}=\mathcal{L}_{4}\left(\phi^{\prime}+\epsilon F\left(\phi^{\prime}\right)\right)+\epsilon \mathcal{L}_{6}\left(\phi^{\prime}+\epsilon F\left(\phi^{\prime}\right)\right)=\mathcal{L}_{4}\left(\phi^{\prime}+\epsilon F\left(\phi^{\prime}\right)\right)+\epsilon \mathcal{L}_{6}\left(\phi^{\prime}\right)+\mathcal{O}\left(\epsilon^{2}\right)$.

By Taylor expanding $\mathcal{L}_{4}$ with respect to $\phi^{\prime}$ and $\partial_{\mu} \phi^{\prime}$ we get:

$$
\mathcal{L}=\mathcal{L}_{4}\left(\phi^{\prime}\right)+\epsilon\left[\frac{\partial \mathcal{L}_{4}}{\partial \phi^{\prime}} F\left(\phi^{\prime}\right)+\frac{\partial \mathcal{L}_{4}}{\partial\left(\partial_{\mu} \phi^{\prime}\right)} \partial_{\mu} F\left(\phi^{\prime}\right)\right]+\epsilon \mathcal{L}_{6}\left(\phi^{\prime}\right)
$$

wich can be rewritten via partial integration to:

$$
\mathcal{L}=\mathcal{L}_{4}\left(\phi^{\prime}\right)+\epsilon\left[\frac{\partial \mathcal{L}_{4}}{\partial \phi^{\prime}}-\partial_{\mu} \frac{\partial \mathcal{L}_{4}}{\partial\left(\partial_{\mu} \phi^{\prime}\right)}\right] F\left(\phi^{\prime}\right)+\epsilon \mathcal{L}_{6}\left(\phi^{\prime}\right) .
$$

It's clear if we take the equations of motion for $\mathcal{L}_{4}$ the term in the brackets will vanish and the physics of the system will be the same as the one we started with in Eq. (6-7). One can conclude that the field redefinition from Eq. (6-8) is equivalent to using the equations of motion up to terms $\mathcal{O}\left(\epsilon^{2}\right)$. This trick function as a change of basis, and will be very useful to simplify our calculations.

Now, going back to our Lagrangian in Eq. (6-6), we can take the equations of motion for $\mathcal{L}_{4}$ :

$$
\begin{gathered}
i\left(\mathbb{1}+Q^{\dagger} Q\right) \not D e_{R}+Y_{e}^{\dagger} H^{\dagger} l_{L}=0 \\
i \not D l_{L}+H Y_{e} e_{R}=0 .
\end{gathered}
$$

However, before applying these equations to the dimension-6 Lagrangian, it is very useful to use partial integration to simplify the calculations:

$$
\mathcal{L}_{6}=-i \bar{e}_{R} Q^{\dagger}\left(M^{\dagger} M\right)^{-1} Q \not D^{3} e_{R}=i \bar{e}_{R} \overleftarrow{I D} Q^{\dagger}\left(M^{\dagger} M\right)^{-1} Q \not D^{2} e_{R}
$$

Then, using the equation of motion

$$
\not D e_{R}=i\left(\mathbb{1}+Q^{\dagger} Q\right)^{-1} Y_{e}^{\dagger} H^{\dagger} l_{L}
$$

our Lagrangian takes the shape:

$\mathcal{L}_{6}=i\left\{-i \bar{l}_{L} H Y_{e}\left[\left(\mathbb{1}+Q^{\dagger} Q\right)^{\dagger}\right]^{-1} Q^{\dagger}\right\}\left(M^{\dagger} M\right)^{-1} Q \not D\left\{i\left(\mathbb{1}+Q^{\dagger} Q\right)^{-1} Y_{e}^{\dagger} H^{\dagger} l_{L}\right\}$.

Since the $\not D$ operator is acting on the last bracket as a whole, by the sum rule, it will give out two terms, one for when it acts upon $H^{\dagger}$ and another one for 
$l_{L}:$

$$
\begin{aligned}
& \mathcal{L}_{6}=i \bar{l}_{L} H Y_{e}\left[\left(\mathbb{1}+Q^{\dagger} Q\right)^{\dagger}\right]^{-1} Q^{\dagger}\left(M^{\dagger} M\right)^{-1} Q\left(\mathbb{1}+Q^{\dagger} Q\right)^{-1} Y_{e}^{\dagger}\left(\not D H^{\dagger}\right) l_{L} \\
& +i \bar{l}_{L} H Y_{e}\left[\left(\mathbb{1}+Q^{\dagger} Q\right)^{\dagger}\right]^{-1} Q^{\dagger}\left(M^{\dagger} M\right)^{-1} Q\left(\mathbb{1}+Q^{\dagger} Q\right)^{-1} Y_{e}^{\dagger} H^{\dagger}\left(\not D l_{L}\right) .
\end{aligned}
$$

Now, we can use the equation of motion

$$
\not D l_{L}=i H Y_{e} e_{R}
$$

on the second term of Eq. (6-13) to get a Yukawa interaction, and thus our Lagrangian will be written as:

$$
\mathcal{L}_{6}=i \bar{l}_{L}^{\alpha i} \Delta_{i j} H_{\alpha} \gamma^{\mu}\left(D_{\mu} H^{\beta}\right)^{*} l_{L}^{\beta j}+\mathcal{L}_{\text {Yukawa }},
$$

where

$$
\Delta_{i j}=\left(\mathbb{1}+Q^{\dagger} Q\right)^{-1} Y_{e} Q^{\dagger}\left(M^{\dagger} M\right)^{-1} Q\left(\mathbb{1}+Q^{\dagger} Q\right)^{-1} Y_{e}^{\dagger}
$$

and

$$
\mathcal{L}_{Y \text { ukawa }}=-\bar{l}_{L}\left(\mathbb{1}+Q^{\dagger} Q\right)^{-1} H Y_{e} Q^{\dagger}\left(M^{\dagger} M\right)^{-1} Q\left(\mathbb{1}+Q^{\dagger} Q\right)^{-1} Y_{e}^{\dagger}|H|^{2} Y_{e} e_{R}
$$

Here $i, j$ and $\alpha, \beta$ are flavour and $\mathrm{SU}(2)$ indices, respectively. Since the $\Delta$ matrix generally have non-zero off diagonal terms, this Lagrangian contains flavour mixing. The leading FCNC processes appear by building a diagram putting together a $\mathcal{L}_{6}$ vertex (lepton $1 \rightarrow$ lepton $\left.2+Z\right)$ with a $\mathcal{L}_{4}$ vertex $(Z \rightarrow$ lepton + lepton) mediated by the exchange of a $\mathrm{Z}$ propagator which comes from the covariant derivative operator $\left(D_{\mu}\right)$. That is exactly what we were looking for.

We are now ready to confront the most general case for any set of $N_{l}, N_{e}$. In the previous chapter, we took the limit $p^{2} \rightarrow 0$ to calculate $Z_{n}$, which gave us

$$
Z_{n}(0)=\sum_{k=0}^{n}\left(Q^{\dagger}\right)^{k} Q^{k}
$$

We will revisit the calculation, but this time around we will keep the subleading terms. Applying a Taylor expansion to $Z_{n}$ in momentum space will result in the dimension- 6 operator that we are looking for. In the Chapter 4 , just before we took the soft limit, our recursion relation for $Z_{N}$ looked like:

$$
Z_{N+1}\left(p^{2}\right)=\mathbb{1}+Q^{\dagger} Z_{N}\left(p^{2}\right)\left[\mathbb{1}-p^{2}\left(M^{\dagger} M\right)^{-1} Z_{N}\left(p^{2}\right)\right]^{-1} Q
$$

where $Z_{0}\left(p^{2}\right)=\mathbb{1}$. By Taylor expanding up to first order in $p^{2}$ :

$$
Z_{N+1}\left(p^{2}\right)=Z_{N+1}(0)+p^{2} Z_{N+1}^{\prime}(0),
$$


where

$$
Z_{N+1}^{\prime}(0)=\left.\frac{\partial}{\partial p^{2}} Z_{N+1}\left(p^{2}\right)\right|_{p^{2}=0} .
$$

We can take $p^{2}=0$ in the Eq. (6-15) and get

$$
Z_{N+1}(0)=\mathbb{1}+Q^{\dagger} Z_{N}(0) Q
$$

This gives exactly what we found in the previous chapter. By taking $\frac{\partial}{\partial p^{2}}$ in Eq. (6-15) one finds:

$$
Z_{N+1}^{\prime}(0)=Q^{\dagger} Z_{N}^{\prime}(0) Q+Q^{\dagger} Z_{N}(0)\left(M^{\dagger} M\right)^{-1} Z_{N}(0) Q
$$

To simplify the notation going forwards we are going to call:

$$
Z_{N}^{\prime}(0) \equiv A_{N}
$$

and

$$
Z_{N}(0) \equiv S_{N}=\sum_{k=0}^{N}\left(Q^{\dagger}\right)^{k} Q^{k}
$$

which leaves us with

$$
Z_{N}=S_{N}+\not D^{2} A_{N}
$$

Thus, one can find a recursion relation for $A_{N}$ by looking at Eq. (6-17):

$$
A_{N+1}=Q^{\dagger}\left(A_{N}+S_{N}\left(M^{\dagger} M\right)^{-1} S_{N}\right) Q,
$$

where $A_{0}=0$. With this result one can find, by induction, an expression for $A_{N}$ :

$$
A_{N}=\sum_{k=0}^{N-1}\left(Q^{\dagger}\right)^{N-k} S_{k}\left(M^{\dagger} M\right)^{-1} S_{k} Q^{N-k} .
$$

Now we have all the operators we need to compute the effective Lagrangian for the Clockwork Model. For Leptons:

$$
\begin{gathered}
\mathcal{L}_{4}^{\text {leptons }}=i \bar{l}_{L} S_{N_{l}} \not D l_{L}+i \bar{e}_{R} S_{N_{e}} \not D e_{R}+\bar{l}_{L} H Y_{e} e_{R}+\bar{e}_{R} Y_{e}^{\dagger} H^{\dagger} l_{L} \\
\mathcal{L}_{6}^{\text {leptons }}=\frac{1}{\mu^{2}} i \bar{l}_{L} \overleftarrow{\not D} \hat{A}_{N_{l}} \not D^{2} l_{L}+\frac{1}{\mu^{2}} i \bar{e}_{R} \overleftarrow{I D} \hat{A}_{N_{e}} \not D^{2} e_{R}
\end{gathered}
$$

where $\mu$ is the typical mass scale of the clockwork leptons, that is $M=\mu \hat{M}$ and $A=\mu^{-2} \hat{A}$. After taking the equations of motion and applying these transformations in order to respect proper normalization:

$$
\begin{array}{ccc}
\bar{e}_{R} \rightarrow \bar{e}_{R} S_{N_{e}}^{-1 / 2} & e_{R} \rightarrow S_{N_{e}}^{-1 / 2} e_{R} & e_{R} \rightarrow U_{e} e_{R}, \\
\bar{l}_{L} \rightarrow \bar{l}_{L} S_{N_{l}}^{-1 / 2} & l_{L} \rightarrow S_{N_{l}}^{-1 / 2} l_{L} & l_{L} \rightarrow U_{l} l_{L},
\end{array}
$$

where $U_{e}$ and $U_{l}$ are rotation matrices that diagonalize the Yukawa. One ends 
up with:

$$
\begin{gathered}
\mathcal{L}_{6}^{\text {leptons }}=\frac{1}{\mu^{2}} i \bar{e}_{R}^{i} \Delta_{i j}^{e} H_{\alpha}^{\dagger} \gamma^{\mu}\left(D_{\mu} H^{\alpha}\right) e_{R}^{j}+\frac{1}{\mu^{2}} i \bar{l}_{L}^{\alpha i} \Delta_{i j}^{l} H_{\alpha} \gamma^{\mu}\left(D_{\mu} H^{\beta}\right)^{*} l_{L}^{\beta j}, \\
\Delta_{e}=U_{e}^{\dagger} S_{N_{e}}^{-1 / 2} Y_{e}^{\dagger}\left(S_{N_{l}}^{\dagger}\right)^{-1} \hat{A}_{N_{l}} S_{N_{l}}^{-1} Y_{e} S_{N_{e}}^{-1 / 2} U_{e} \\
\Delta_{l}=U_{l}^{\dagger} S_{N_{l}}^{-1 / 2} Y_{e}\left(S_{N_{e}}^{\dagger}\right)^{-1} \hat{A}_{N_{e}} S_{N_{e}}^{-1} Y_{e}^{\dagger} S_{N_{l}}^{-1 / 2} U_{l},
\end{gathered}
$$

where $i, j$ and $\alpha, \beta$ are flavour and $\mathrm{SU}(2)$ indices, respectively. We also omitted the Yukawa terms as their contribution is neglectable. For quarks, this process is entirely analogous, the result is as shown below:

$$
\begin{gathered}
\mathcal{L}_{6}^{q u a r k s}=\frac{1}{\mu^{2}} i \bar{u}_{R}^{i} \Delta_{i j}^{u} \tilde{H}_{\alpha}^{\dagger} \gamma^{\mu}\left(D_{\mu} \tilde{H}^{\alpha}\right) u_{R}^{j}+\frac{1}{\mu^{2}} i \bar{d}_{R}^{i} \Delta_{i j}^{d} H_{\alpha}^{\dagger} \gamma^{\mu}\left(D_{\mu} H^{\alpha}\right) d_{R}^{j} \\
+\frac{1}{\mu^{2}} i \bar{q}_{L}^{\alpha i} \Delta_{i j}^{q_{u}} \tilde{H}_{\alpha} \gamma^{\mu}\left(D_{\mu} \tilde{H}^{\beta}\right)^{*} q_{L}^{\beta j}+\frac{1}{\mu^{2}} i \bar{q}_{L}^{\alpha i} \Delta_{i j}^{q_{d}} H_{\alpha} \gamma^{\mu}\left(D_{\mu} H^{\beta}\right)^{*} q_{L}^{\beta j} \\
\Delta_{u}=U_{u}^{\dagger} S_{N_{u}}^{-1 / 2} Y_{u}^{\dagger}\left(S_{N_{q}}^{\dagger}\right)^{-1} \hat{A}_{N_{q}} S_{N_{q}}^{-1} Y_{u} S_{N_{u}}^{-1 / 2} U_{u} \\
\Delta_{d}=U_{d}^{\dagger} S_{N_{d}}^{-1 / 2} Y_{d}^{\dagger}\left(S_{N_{q}}^{\dagger}\right)^{-1} \hat{A}_{N_{q}} S_{N_{q}}^{-1} Y_{d} S_{N_{d}}^{-1 / 2} U_{d} \\
\Delta_{q_{u}}=U_{q}^{\dagger} S_{N_{q}}^{-1 / 2} Y_{u}\left(S_{N_{u}}^{\dagger}\right)^{-1} \hat{A}_{N_{u}} S_{N_{u}}^{-1} Y_{u}^{\dagger} S_{N_{q}}^{-1 / 2} U_{q} \\
\Delta_{q_{d}}=U_{q}^{\dagger} S_{N_{q}}^{-1 / 2} Y_{d}\left(S_{N_{d}}^{\dagger}\right)^{-1} \hat{A}_{N_{d}} S_{N_{d}}^{-1} Y_{d}^{\dagger} S_{N_{q}}^{-1 / 2} U_{q}
\end{gathered}
$$

where $i, j$ and $\alpha, \beta$ are flavour and $\mathrm{SU}(2)$ indices, respectively. Here, one must recall that the physical Yukawas corresponds to:

$$
\tilde{Y}_{e}=S_{N_{l}}^{-\frac{1}{2}} Y_{e} S_{N_{e}}^{-\frac{1}{2}}, \quad \tilde{Y}_{u}=S_{N_{q}}^{-\frac{1}{2}} Y_{u} S_{N_{u}}^{-\frac{1}{2}}, \quad \tilde{Y}_{d}=S_{N_{q}}^{-\frac{1}{2}} Y_{d} S_{N_{d}}^{-\frac{1}{2}},
$$

which can be diagonalized as

$$
\begin{aligned}
& \tilde{Y}_{e}=U_{l}\left(\begin{array}{lll}
y_{e} & & \\
& y_{\mu} & \\
& & y_{\tau}
\end{array}\right) U_{e}^{\dagger}, \\
& \tilde{Y}_{u}=U_{q}\left(\begin{array}{lll}
y_{u} & & \\
& y_{c} & \\
& & \\
& & y_{t}
\end{array}\right) U_{u}^{\dagger}, \\
& \tilde{Y}_{d}=U_{l}\left(\begin{array}{lll}
y_{d} & & \\
& y_{s} & \\
& & y_{b}
\end{array}\right) U_{d}^{\dagger} .
\end{aligned}
$$


The effective Lagrangian from Eq. (6-24) and Eq. (6-27) contains several processes which can be used to put a constraint on our model. The next step is to pick a relevant process, compute its prediction in our model and compare it with the Particle Data Group (PDG) database. This way, we may impose a limit on the mass scale, $\mu$.

\section{2}

\section{The $\mathcal{L}_{6}$ operators}

In this subsection we take a deeper look into the dimension- 6 operators present in our EFT and the processes that it depicts in order to choose, among those processes, the best suited to proceed with the analysis through simulation. At first glance, we can see, from the Lagrangian, that, at tree level, there are two possible ways to build a FCNC diagram, either by taking a $\mathcal{L}_{6}$ vertex and connect with a $\mathcal{L}_{4}$ vertex via an exchange of a virtual $\mathrm{Z}$ boson, or by taking two $\mathcal{L}_{6}$ vertices instead. The important difference between these two options is that in the case where we take two EFT vertices there can be an occurrence of two flavour changing decays (when this happens, we say that this diagram has a $\Delta F=2$, which stands for the double flavour changing) when in the other case the flavour changing appears in only one vertex $(\Delta F=1)$. Since the dimension- 6 vertex is suppressed by a cutoff scale, it is easy to see that the $\Delta F=2$ process will occur at a much lower probability, being so we can infer that we should prioritize processes with a single flavour changing appearance. There are a few processes in the literature that can be found within our EFT, among those some that we consider for this study are presented in Table 6.1 and the related upper bounds for the Branching Ratios (taken from (7)) are presented in Table 6.2. We will take a look at each of those processes one by one and by the end, based on the features presented, select one as the most suitable for our simulation run.

Table 6.1: FCNC processes in the effective Clockwork model.

\begin{tabular}{ccc}
\hline$\Delta F$ & Tree level & 1 Loop \\
\hline 1 & $\mu \rightarrow e e e$ & $\mu \rightarrow e \gamma, b \rightarrow s \gamma$ \\
2 & $K-\bar{K}$ & \\
\hline
\end{tabular}

\subsection{1}

$\mu \rightarrow$ eee

We start with this muon decay mode as it showcases the most obvious choice, being the only tree level process with $\Delta F=1$ in our model. The other 
Table 6.2: Upper bounds for FCNC Branching Ratios.

\begin{tabular}{lc}
\hline Process & $B R$ \\
\hline$\mu \rightarrow e e e$ & $<1.0 \times 10^{-12}(7)$ \\
$\mu \rightarrow e \gamma$ & $<4.2 \times 10^{-13}(7)$ \\
$b \rightarrow s \gamma$ & $=3.55 \times 10^{-4}$ (experimental) $(76)$ \\
$b \rightarrow s \gamma$ & $=2.98 \times 10^{-4}$ (theoretical) $(77)$ \\
\hline
\end{tabular}

processes with $\Delta F=2$ tends to suffer from a greater suppression by higher powers of $\frac{v}{\mu}$, where $\mu \sim 100 \mathrm{TeV}$ is the clockwork fermion typical mass scale later found from simulations, while the 1 loop ones are suppressed by a factor of $\frac{e^{2}}{16 \pi^{2}}$. Diagrams with more than one loop are immediately disconsidered, as any additional loop would just further increase the suppression by picking up extra factors of $\frac{e^{2}}{16 \pi^{2}}$. In any case, it is important to confirm such statement by also taking a careful look at the remaining options.

A possible diagram for this decay is presented in Fig. 6.1. The dimension6 vertex can be taken from the Lagrangian of Eq. (6-24), while the other vertex is taken from the SM Lagrangian. This way one can estimate the amplitude of the diagram as

$$
\mathcal{M}_{\mu \rightarrow e e e} \sim \frac{v^{2}}{\mu^{2}} \frac{1}{m_{Z}^{2}}\left(\bar{e}_{R} \Delta_{12}^{e} \mu_{R}+\bar{e}_{L} \Delta_{12}^{l} \mu_{L}\right) .
$$

One can do an analogous procedure to estimate the amplitude for each of the next processes considered and, by means of comparison, see which one is most likely to be produced in our effective Lagrangian.

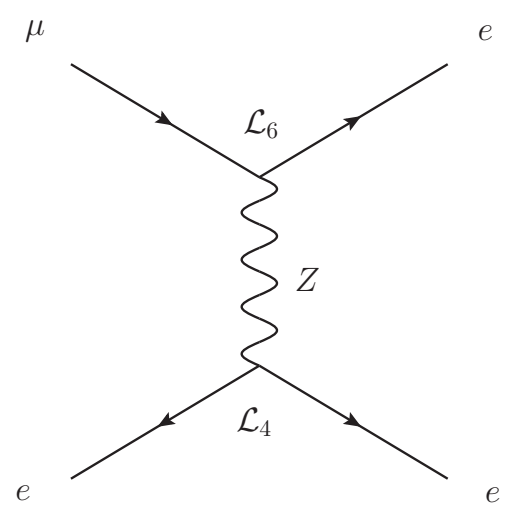

Figure 6.1: Feynman diagram for $\mu \rightarrow e e e$. This a diagram is composed by a dimension- 6 and dimension- 4 vertices. 


\subsection{2}

$\mu \rightarrow e \gamma$

This diagram is composed by one loop and a $\mathcal{L}_{6}$ operator insertion, like it is demonstrated in Fig. 6.2. The process depicted here comes from the dimension-5 operator

$$
\mathcal{L}_{\text {eff }}=\frac{1}{\Lambda} F_{\mu \nu} \bar{e}_{R} \gamma^{\mu \nu} \mu_{L}
$$

where $\Lambda$ is the is the cutoff scale for the EFT. Since the diagram in Fig. 6.2 must be proportional to $\mu^{-2}$, one can use dimensional analysis to estimate

$$
\frac{1}{\Lambda^{2}} \sim \frac{e^{2}}{\left(16 \pi^{2}\right)^{2}} \frac{\left(\left|\Delta_{12}^{e}\right|^{2}+\left|\Delta_{12}^{l}\right|^{2}\right)}{\mu^{4}} m_{\mu}^{2}
$$

The decay rate of this mode is given by

$$
\Gamma(\mu \rightarrow e \gamma)=\frac{1}{2 \pi} \frac{1}{\Lambda^{2}} m_{\mu}^{3} .
$$

Since the BR is given by

$$
B R(\mu \rightarrow e e e)=\frac{\Gamma(\mu \rightarrow e e e)}{\Gamma(\mu \rightarrow e \nu \nu)} \quad, \quad \Gamma(\mu \rightarrow e \nu \nu)=\frac{G_{F}^{2} m_{\mu}^{5}}{192 \pi^{3}},
$$

taking Eq. (6-39) and Eq. (6-38) as inputs for Eq. (6-40) allow us to estimate the BR for this decay as

$$
B R(\mu \rightarrow e \gamma) \sim \frac{16 e^{2}}{3 \pi^{2}}\left(\left|\Delta_{12}^{e}\right|^{2}+\left|\Delta_{12}^{l}\right|^{2}\right) \frac{v^{4}}{\mu^{4}}
$$

Here the factor of $\frac{v^{4}}{\mu^{4}}$ greatly suppresses this diagram, since the mass scale $\mu$ is expected to be orders of magnitude larger than the scale $v$. Additionally, if one recalls the value for the fine-structure constant,

$$
\alpha=\frac{e^{2}}{4 \pi} \approx \frac{1}{137}
$$

it becomes very clear why this diagram is heavily suppressed. Even tough the upper bound for this process would be more interesting for our purposes, as it would, in theory, when looking only at the upper bound of the $B R$, be able to produce a more refined constraint on the typical clockwork mass scale, when also taking into account the suppression in its amplitude it ends up surpassing the attractiveness of its $B R$. Being so, one can safely eliminate this decay mode from the selection of the process that is most suitable for the computational analysis. 


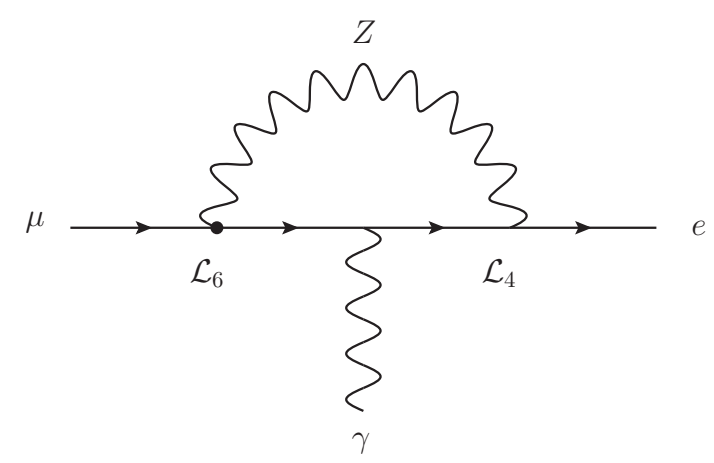

Figure 6.2: Feynman diagram for $\mu \rightarrow e \gamma$. This diagram is heavily suppressed by the loop correction factor.

\subsection{3}

$b \rightarrow s \gamma$

This process occurs when a $B$ meson decays into a strange flavoured meson plus a photon and its diagram is depicted in Fig. 6.3. While this process is not actually forbidden in the SM, as it just happens to be a very rare decay mode of the $B$ mesons, there are still a small deviation between the BR measured experimentally and the BR computed by theory (roughly 10\%). This minor discrepancy can come from some new physics as represented by:

$$
\frac{B R_{N P}(b \rightarrow s \gamma)-B R_{S M}(b \rightarrow s \gamma)}{B R_{S M}(b \rightarrow s \gamma)}<\frac{\sigma_{S M}(b \rightarrow s \gamma)}{B R_{S M}(b \rightarrow s \gamma)},
$$

where NP stands for new physics and $\sigma$ is the difference between the experimental data and the theoretical calculation. Since for any decay

$$
B R \sim|\mathcal{M}|^{2}
$$

and, within the framework of the clockwork model, we can take

$$
\mathcal{M}_{N P}=\mathcal{M}_{C W}+\mathcal{M}_{S M},
$$

where $C W$ stands for Clockwork, we can rewrite Eq. (6-42) as

$$
\frac{\left|\mathcal{M}_{S M}\right|^{2}+\mathcal{M}_{S M}^{\dagger} \mathcal{M}_{C W}+\mathcal{M}_{C W}^{\dagger} \mathcal{M}_{S M}+\left|\mathcal{M}_{C W}\right|^{2}-\left|\mathcal{M}_{S M}\right|^{2}}{\left|\mathcal{M}_{S M}\right|^{2}}<\frac{\sigma_{S M}}{B R_{S M}} .
$$

However, since the contribution from $\left|\mathcal{M}_{C W}\right|^{2}$ is neglectable and, this way, one can rewrite Eq. (6-43) as

$$
2 \frac{\left|\mathcal{M}_{C W}\right|}{\left|\mathcal{M}_{S M}\right|} \sim\left(\frac{v}{\mu}\right)^{2} \frac{\left(\Delta_{23}^{e}+\Delta_{23}^{l}\right)}{V_{C K M}^{c b}}<\frac{\sigma_{S M}}{B R_{S M}} \sim 10 \% .
$$

Now, the lower bound that Eq. (6-44) gives us does not seem to be very useful as the constraint that comes from it is not powerful enough and clearly, by 
looking at Eq. (6-36), one can obtain a much more refined limit for the mass scale $\mu$ by choosing to analyze the muon decay $\mu \rightarrow$ eee instead. For this reason this process can be safely disregarded for our purposes.

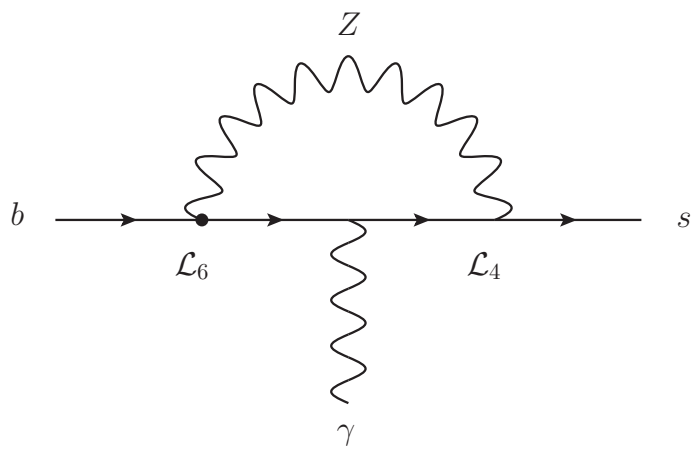

Figure 6.3: Feynman diagram for $b \rightarrow s \gamma$. This decay mode is heavily suppressed due to the 1-loop quantum corrections.

\subsection{4}

\section{$K-\bar{K}$ mixing}

This process has historical significance, as it was responsible for the first measurement of $C P$ violation, when the $K=\bar{s} d$ meson, named kaon, oscillates through strangeness-violating weak interactions to its $C P$ conjugate $\bar{K}=\bar{d} s$. One can take the product of two dimension-6 operators from Eq. (6-27) in order to build an estimation for the related amplitude. One then, finds

$$
\mathcal{M}_{\bar{s} d \rightarrow \bar{d} s} \sim \frac{v^{4}}{\mu^{4}}
$$

Since the mass scale $\mu$ is expected to be around the $100 \mathrm{TeV}$ scale, while $v=246 \mathrm{GeV}$, the factor of $\frac{v^{4}}{\mu^{4}} \sim 4 \times 10^{-11}$ greatly suppresses this process, and so, one can disregard this diagram entirely from the analysis, without any repercussion. 


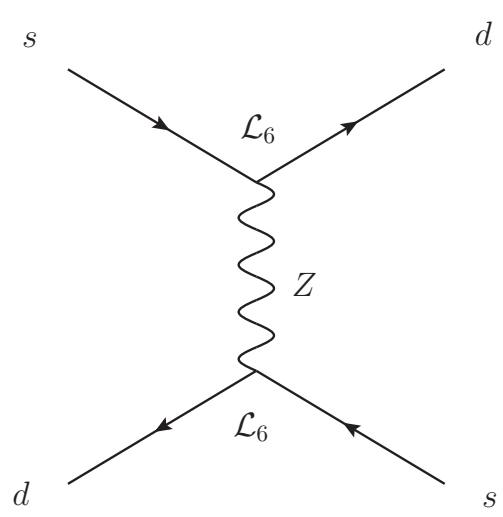

Figure 6.4: Feynman diagram for $K-\bar{K}$ mixing. This diagram is build from the insertion of two dimension- 6 operators which configurates two flavour changings $(\Delta F=2)$.

\subsection{5}

\section{Choosing the right process for computational analysis}

It became very clear, after taking a deeper look at the estimate for the amplitudes of each of the considered process, that the most suitable process to pick for analysis is indeed the muon decay $\mu \rightarrow$ eee, as one could have easily predicted just by noticing that it was the only possible tree level process built with only one $\mathcal{L}_{6}$ vertex and any addition of such vertices would imply in a greater suppression. An analogous argument can be made for the looped processes, as they occur in a loop correction factor of the order of $\frac{1}{137}$ that also causes a suppression of the amplitude. Being so, one can feel fairly confident by putting and end to the search at this point and move to the calculation of the branching ratio for the muon decay mode $\mu \rightarrow$ eee in our model in order to find a lower bound for the clockwork fermion typical mass scale $\mu$.

\section{3}

\section{Muon decay}

In this subsection we will compute the Branching Ratio (BR) for the muon decay $(\mu \rightarrow$ eee $)$ in order to find a limit for a typical mass scale of the clockwork leptons. There are two possible diagrams for this process, as shown in Fig. 6.5.

The vertices of the diagrams are the following:

$$
\begin{aligned}
\mathcal{L}_{6}^{\mu \rightarrow e Z}=\frac{v^{2}}{4 \mu^{2}}\left[\bar{e}_{R} \Delta_{12}^{e}(\right. & \left.\left.g_{1} \sin \theta+g_{2} \cos \theta\right) \mathcal{Z} \mu_{R}\right] \\
& -\frac{v^{2}}{4 \mu^{2}}\left[\bar{e}_{L} \Delta_{12}^{l}\left(g_{1} \sin \theta+g_{2} \cos \theta\right) \boldsymbol{中} \mu_{L}\right]+\text { h.c. },
\end{aligned}
$$




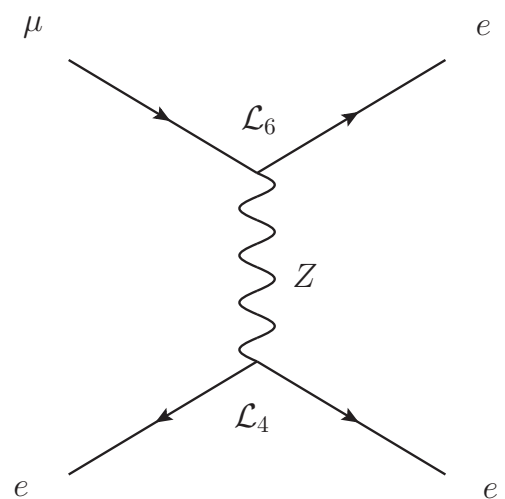

(a) t-channel

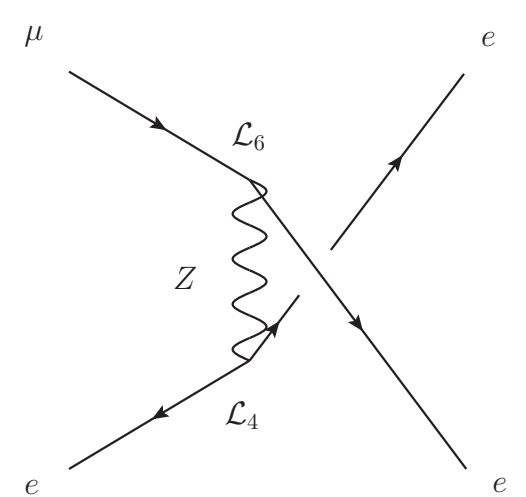

(b) u-channel

Figure 6.5: Feynman diagrams for the muon decay. There are two possible diagrams.

$$
\mathcal{L}_{4}^{Z \rightarrow e e}=-\bar{e}_{R} g_{1} \sin \theta \not{Z} e_{R}-\bar{e}_{L}\left(\frac{g_{1}}{2} \sin \theta+\frac{g_{2}}{2} \cos \theta\right) \not e_{L},
$$

and the $\mathrm{Z}$ propagator:

$$
G_{Z}^{\mu \nu}=-\frac{i\left(g^{\mu \nu}-\frac{p^{\mu} p^{\nu}}{m_{Z}^{2}}\right)}{p^{2}-m_{Z}^{2}} .
$$

To build our EFT we had to take the limit where $p^{2}$ is small, so it is appropriate to take the limit $p^{2} \ll m_{Z}^{2}$ here to simplify our calculations. This is analogous to the limit taken in the Fermi Theory. In this way the propagator line is reduced to a point and the two vertices are joint together. The resulting diagram is the one shown in Fig. 6.6.

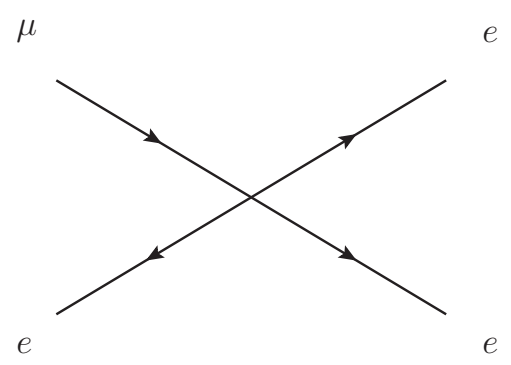

Figure 6.6: EFT Feynman diagram. Here the $Z$ propagator was broken down to a single 4-fermion interaction vertex

This diagram is easy to calculate, and gives us:

$$
\begin{aligned}
& \mathcal{M}_{t}=\frac{v^{2}}{4 \mu^{2}} \frac{\left(g_{1} \sin \theta+g_{2} \cos \theta\right)}{m_{Z}^{2}}\left[\Delta_{12}^{l} \bar{e}_{L} \gamma^{\mu} \mu_{L}-\Delta_{12}^{e} \bar{e}_{R} \gamma^{\mu} \mu_{R}\right]\left[\left(\frac{g_{1}}{2} \sin \theta\right.\right. \\
& \left.\left.+\frac{g_{2}}{2} \cos \theta\right) \bar{e}_{L} \gamma^{\mu} e_{L}+g_{1} \sin \theta \bar{e}_{R} \gamma^{\mu} e_{R}\right] \text {. }
\end{aligned}
$$

Assuming that the spin of the particles is not measured we need to average over the spins of the muon and sum over the spins of the eletrons in order to 
compute the full amplitude. The total amplitude squared is given by:

$$
|\mathcal{M}|^{2}=\left|\mathcal{M}_{t}\right|^{2}+\mathcal{M}_{u} \mathcal{M}_{t}^{\dagger}+\mathcal{M}_{t} \mathcal{M}_{u}^{\dagger}+\left|\mathcal{M}_{u}\right|^{2}
$$

where the amplitude for the u-channel diagram can be obtained by taking the result for $\mathcal{M}_{t}$ and simply swapping $p_{2}$ and $p_{4}$ and then apply the Fierz identity (78). The full calculation of the amplitudes, although straightforward, has a few minor mathematical challenges that can be overcome by employing some useful identities, such as the spinor identities, the gamma matrices identities and the Fierz identity. The identities are presented below:

Spinor identities:

$$
\sum_{s} u_{s}(p) \bar{u}_{s}(p)=\not p+m \quad ; \quad \sum_{s} v_{s}(p) \bar{v}_{s}(p)=\not p-m,
$$

where $u$ and $v$ are spinors for particle and antiparticle, respectively and $s$ is the spin.

Gamma matrices identities:

$$
\begin{gathered}
\left\{\gamma^{5}, \gamma^{\mu}\right\}=0 \\
\operatorname{Tr}\left(\gamma^{\mu}\right)=\operatorname{Tr}\left(\gamma^{\alpha} \gamma^{\beta} \gamma^{\mu}\right)=0 \\
\operatorname{Tr}\left(\gamma^{\mu} \gamma^{\nu}\right)=4 g^{\mu \nu} \\
\operatorname{Tr}\left(\gamma^{\alpha} \gamma^{\mu} \gamma^{\beta} \gamma^{\nu}\right)=4\left(g^{\alpha \mu} g^{\beta \nu}-g^{\alpha \beta} g^{\mu \nu}+g^{\alpha \nu} g^{\beta \mu}\right), \\
\operatorname{Tr}\left(\gamma^{5}\right)=\operatorname{Tr}\left(\gamma^{\mu} \gamma^{5}\right)=\operatorname{Tr}\left(\gamma^{\alpha} \gamma^{\mu} \gamma^{\beta} \gamma^{5}\right)=0, \\
\operatorname{Tr}\left(\gamma^{\alpha} \gamma^{\mu} \gamma^{\beta} \gamma^{\nu} \gamma^{5}\right)=-4 i \epsilon^{\alpha \mu \beta \nu}
\end{gathered}
$$

Antisymmetric tensor identities:

$$
\begin{gathered}
\epsilon^{\alpha \mu \beta \nu} \epsilon_{\alpha^{\prime} \mu \beta^{\prime} \nu}=2\left(\delta_{\alpha^{\prime}}^{\alpha} \delta_{\beta^{\prime}}^{\beta}-\delta_{\beta^{\prime}}^{\alpha} \delta_{\alpha^{\prime}}^{\beta}\right), \\
p_{1}^{\alpha} p_{2}^{\beta} p_{3}^{\mu} p_{4}^{\nu} \epsilon_{\alpha \beta \mu \nu}=0 \quad, \quad\left(\text { if } p_{1}^{\alpha}=p_{2}^{\beta}+p_{3}^{\mu}+p_{4}^{\nu}\right), \\
p_{1}^{\alpha} p_{2}^{\beta} p_{3}^{\lambda} p_{4}^{\lambda} \epsilon_{\alpha \beta \mu \nu} g^{\mu \nu}=0,
\end{gathered}
$$

where $p_{i}$ is the momentum of particle $i$. 
Fierz identity:

$$
\begin{aligned}
\left(\bar{\chi} \gamma^{\mu} \psi\right)\left(\bar{\psi}^{\prime} \gamma_{\mu} \chi^{\prime}\right)=\left(\bar{\chi} \chi^{\prime}\right) & \left(\bar{\psi}^{\prime} \psi\right)-\frac{1}{2}\left(\bar{\chi} \gamma^{\mu} \chi^{\prime}\right)\left(\bar{\psi}^{\prime} \gamma_{\mu} \psi\right) \\
& -\frac{1}{2}\left(\bar{\chi} \gamma^{\mu} \gamma_{5} \chi^{\prime}\right)\left(\bar{\psi}^{\prime} \gamma_{\mu} \gamma_{5} \psi\right)-\left(\bar{\chi} \gamma_{5} \chi^{\prime}\right)\left(\bar{\psi}^{\prime} \gamma_{5} \psi\right)
\end{aligned}
$$

where $\chi$ and $\psi$ are spinors.

The Particle Data Group has an expression for the three-body decay rate (79) that is very useful in this instance:

$$
d \Gamma=\frac{1}{(2 \pi)^{3}} \frac{1}{8 m_{\mu}}|\overline{\mathcal{M}}|^{2} d E_{2} d E_{3}
$$

where $m_{\mu}$ is the muon mass, $|\overline{\mathcal{M}}|^{2}$ is the amplitude squared averaged over the spins and $E_{2}$ and $E_{3}$ are the energies of the electron and the positron, respectively. The $\mathrm{BR}$ is given by:

$$
B R(\mu \rightarrow e e e)=\frac{\Gamma(\mu \rightarrow e e e)}{\Gamma(\mu \rightarrow e \nu \nu)} \quad, \quad \Gamma(\mu \rightarrow e \nu \nu)=\frac{G_{F}^{2} m_{\mu}^{5}}{192 \pi^{3}},
$$

where $G_{F}$ is the Fermi coupling constant and $m_{\mu}$ is the mass of the muon. After applying the identities listed above and integrating the amplitude squared over the energies, The result for the BR is the following:

$$
B R(\mu \rightarrow \text { eee })=\left(\frac{v}{\mu}\right)^{4} \frac{1}{8}\left[\left|\Delta_{12}^{e}\right|^{2}+4\left(1-\frac{m_{W}^{2}}{m_{Z}^{2}}\right)^{2}\left|\Delta_{12}^{l}\right|^{2}\right],
$$

where $v$ is the Higgs vacuum expectation value after symmetry breaking and $m_{W}, m_{Z}$ are the masses of the $\mathrm{W}$ and $\mathrm{Z}$ bosons, respectively. Is worth noting that, since $\frac{m_{W}}{m_{Z}} \approx 0.88$, one might expect a lesser contribution from $\Delta_{12}^{l}$ and a higher contribution from $\Delta_{12}^{e}$ in the simulation for the BR. However what occurs is quite the opposite, as, it will soon be explained, in general, $\Delta_{12}^{l} \gg \Delta_{12}^{e}$.

\subsection{1}

\section{Muon decay simulation}

The simulation run has set the pair of matrices $M, Q$ as random complex matrices of order 1 and used the previously tuned values for $N_{l}, N_{e}$ and $C_{e}(2$, $9,0.02$, respectively) and plotted the distribution of the BR over ten thousand runs. We then found the value for the clockwork mass scale $\mu$ that best fit the experimental data for the muon decay

$$
B R_{\text {exp }}(\mu \rightarrow e e e)<1.0 \times 10^{-12} .
$$


The procedure used was to at first run a simulation for an auxiliary distribution

$$
B R^{\prime}(\mu \rightarrow \text { eee })=\frac{1}{8}\left[\left|\Delta_{12}^{e}\right|^{2}+4\left(1-\frac{m_{W}^{2}}{m_{Z}^{2}}\right)^{2}\left|\Delta_{12}^{l}\right|^{2}\right],
$$

and then multiply this auxiliary distribution by a small parameter

$$
\epsilon^{4}=\left(\frac{v}{\mu}\right)^{4}
$$

such that it acts as a shift in the distribution and brings the distribution much closer to the experimental value for the BR. This procedure allow us to adjust the distribution and find the best fit value for the parameter $\epsilon$ such that $95 \%$ of the points in the shifted distribution are below the experimental data, that is:

$$
B R_{95 \%}=\epsilon^{4} B R^{\prime}<B R_{e x p}(\mu \rightarrow e e e)=1.0 \times 10^{-12} .
$$

Then we can take a lower limit for the mass scale $\mu$ given by:

$$
\mu>v\left(\frac{B R^{\prime}}{B R_{\exp }}\right)^{1 / 4} \sim 246 \times 10^{3}\left(B R^{\prime}\right)^{1 / 4} \mathrm{GeV} .
$$

This expression already gives an expectation that $\mu$ should be of the order a few hundred $\mathrm{TeV}$, which is quite large.

The main result obtained is a value for $\mu \sim 100 \mathrm{TeV}$, which confirms our expectation of a great suppression of the EFT. The distributions for BR, $\Delta_{12}^{e}$ and $\Delta_{12}^{l}$ are presented in Fig. 6.7 and Fig. 6.8 clearly demonstrate that $B R^{\prime}$ and $\left|\Delta_{12}^{l}\right|^{2}$ have a very similar distribution, which confirms our expectations that the BR has a very small dependence on $\Delta_{12}^{e}$, as it depends mainly on $\Delta_{12}^{l}$.

Another interesting result is the plot $B R \times \frac{m_{e}}{m_{\mu}}$, shown in Fig. 6.9, which reveals a small correlation between $B R$ and $\frac{m_{e}}{m_{\mu}}$ for small values of $B R$. This small correlation can be explained by the fact that, by construction of the clockwork mechanism, the interactions between fermions are exponentially suppressed, that is:

$$
Z_{x}^{-\frac{1}{2}} \sim\left(\begin{array}{ccc}
\tilde{z}_{1}^{x} & & \\
& \tilde{z}_{2}^{x} & \\
& & \tilde{z}_{3}^{x}
\end{array}\right) \Longrightarrow\left\{\begin{array}{c}
\Delta_{12}^{e} \sim \tilde{z}_{1}^{e} \tilde{z}_{2}^{e} \\
\Delta_{12}^{l} \sim \tilde{z}_{1}^{l} \tilde{z}_{2}^{l}
\end{array},\right.
$$

where $x$ can be $e$ or $l$, for the right handed singlet and the left handed doublet of $S U(2)$, respectively, and $\tilde{z}_{i}$ are the eigenvalues of $Z_{x}^{-\frac{1}{2}}$ and, as we have seen, typically one has $z_{1} \ll 1, z_{2}<1$ and $z_{3} \sim 1$. The mechanism also leads to suppressed Yukawas by similar factors of $\tilde{z}$ :

$$
y_{e} \sim \tilde{z}_{1}^{l} \tilde{z}_{1}^{e}, \quad y_{l} \sim \tilde{z}_{2}^{l} \tilde{z}_{2}^{e}
$$


In the previous chapter we have seen that a greater number of clockwork fermions generates a greater suppression on the interactions and on the Yukawas, this implies that:

$$
N_{e} \gg N_{l} \quad \Longrightarrow \quad \tilde{z}_{i}^{e} \ll \tilde{z}_{i}^{l}
$$

This is the case of our simulation. This observation is very useful, as it allow us to take out the factors of $\tilde{z}_{i}^{l}$ from the Yukawa relations on Eq. (6-70), given that the contribution from $\tilde{z}_{i}^{e}$ is much greater. The simplified Yukawa relations are:

$$
y_{e} \sim \tilde{z}_{1}^{e} \quad, \quad y_{l} \sim \tilde{z}_{2}^{e}
$$

Taking a look at the expression for the branching ratio on Eq. (6-64), we can see that it goes as $B R \sim\left|\Delta_{12}^{e}\right|^{2}$ while the mass ratio goes as $\frac{m_{e}}{m_{\mu}} \sim \frac{y_{e}}{y_{\mu}}$. The small correlation between $B R$ and $\frac{m_{e}}{m_{\mu}}$ becomes clear once one rewrites the expression for the branching ratio as factors of $\tilde{z}$ taken from Eq. (6-69):

$$
B R \sim\left(\tilde{z}_{1}^{e}\right)^{2}\left(\tilde{z}_{2}^{e}\right)^{2}
$$

$$
\frac{m_{e}}{m_{\mu}} \sim \frac{\tilde{z}_{1}^{e}}{\tilde{z}_{2}^{e}}
$$




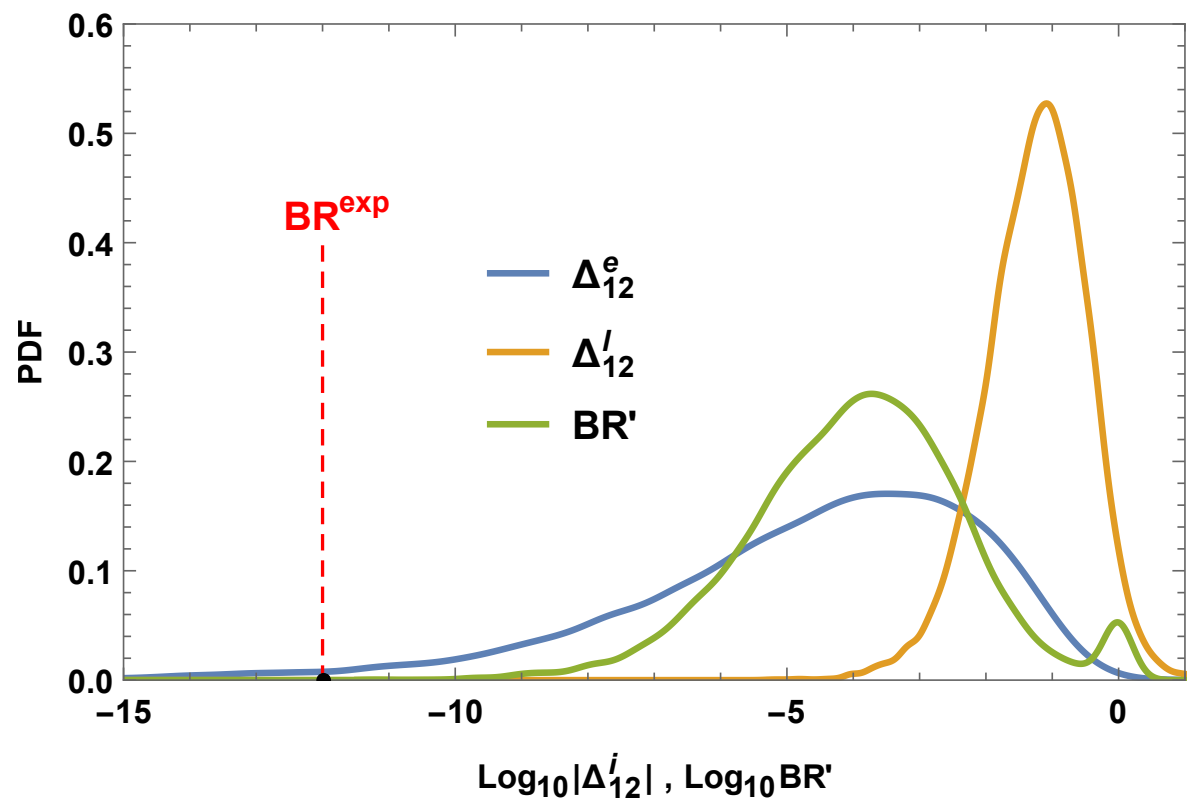

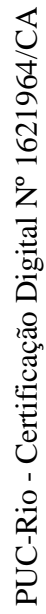

(a) Unshifted distributions

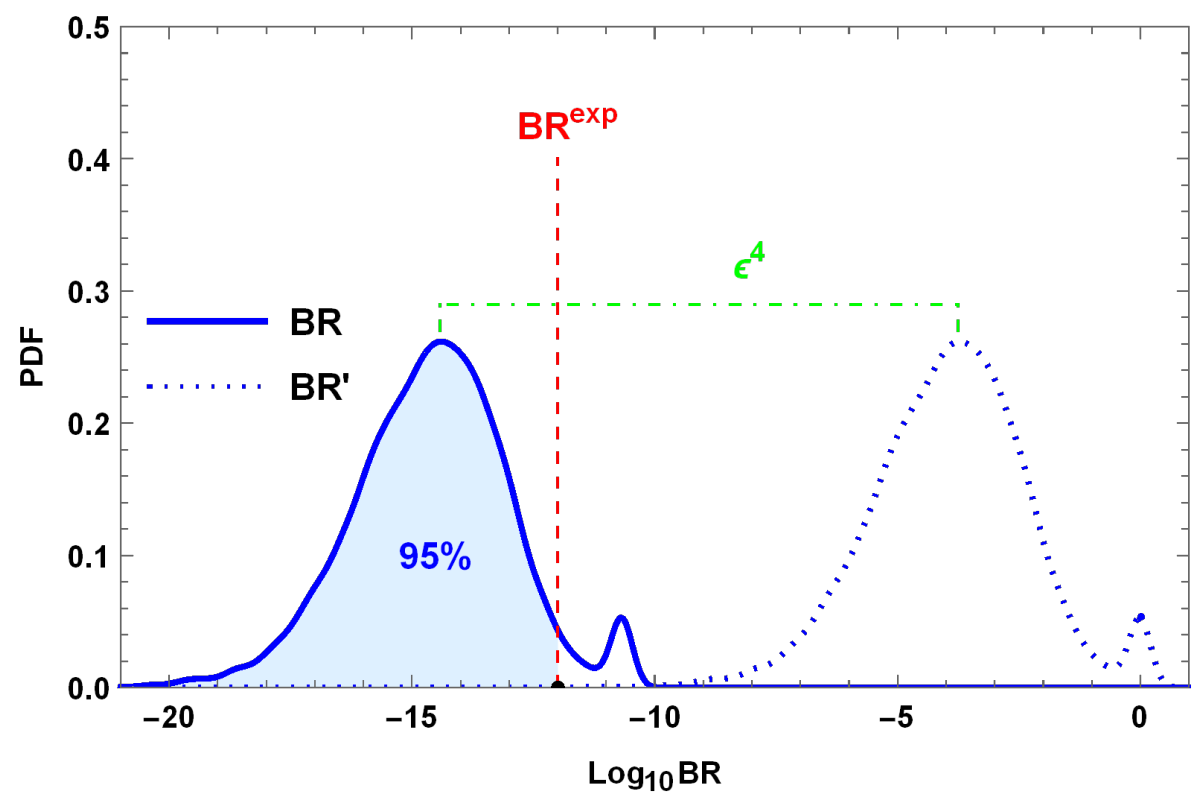

(b) Shifted BR distribution

Figure 6.7: BR distributions. Here the parameter $\epsilon^{4}$ is responsible for shifting the distribution to the point where $95 \%$ of the values are below the experimental value for the BR. 


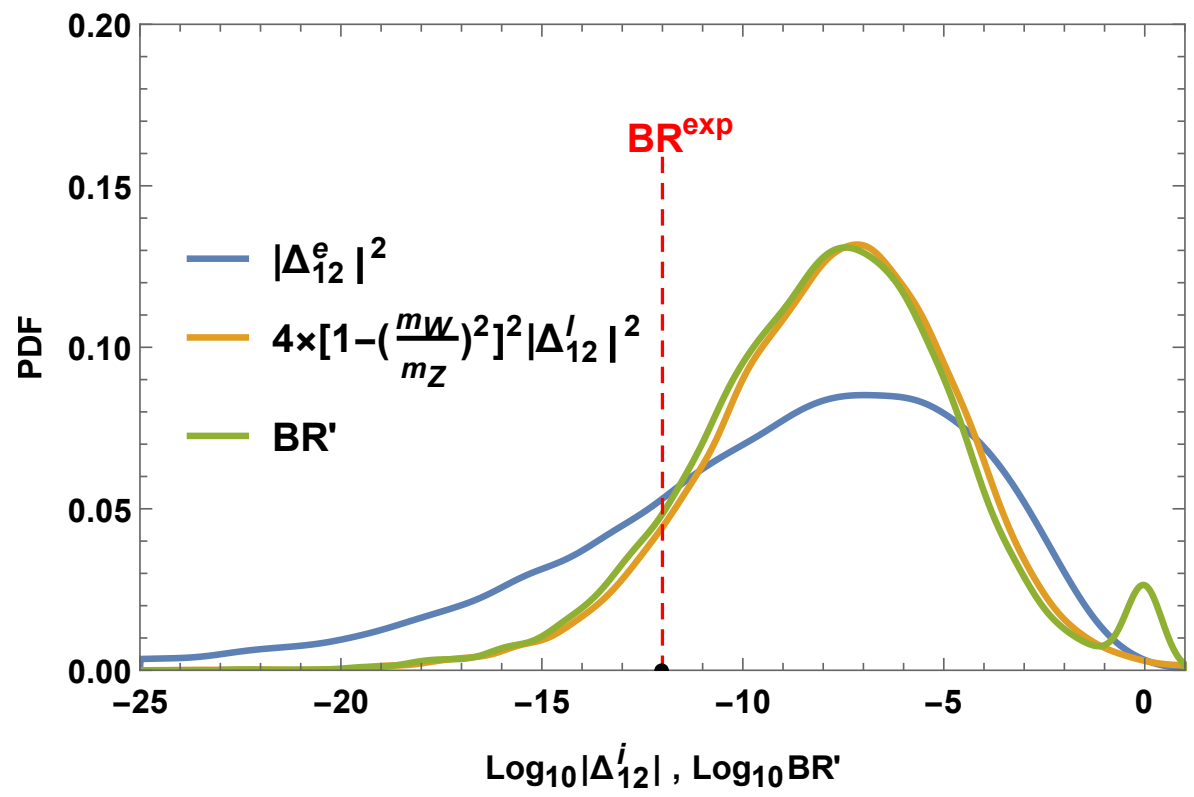

Figure 6.8: Distributions for $B R^{\prime}$ and $\left|\Delta_{12}^{i}\right|^{2}$. Here it becomes clear that $B R^{\prime}$

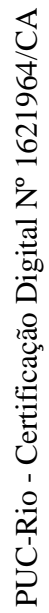
is heavily dependent on $\left|\Delta_{12}^{l}\right|^{2}$ and much less to $\left|\Delta_{12}^{e}\right|^{2}$.

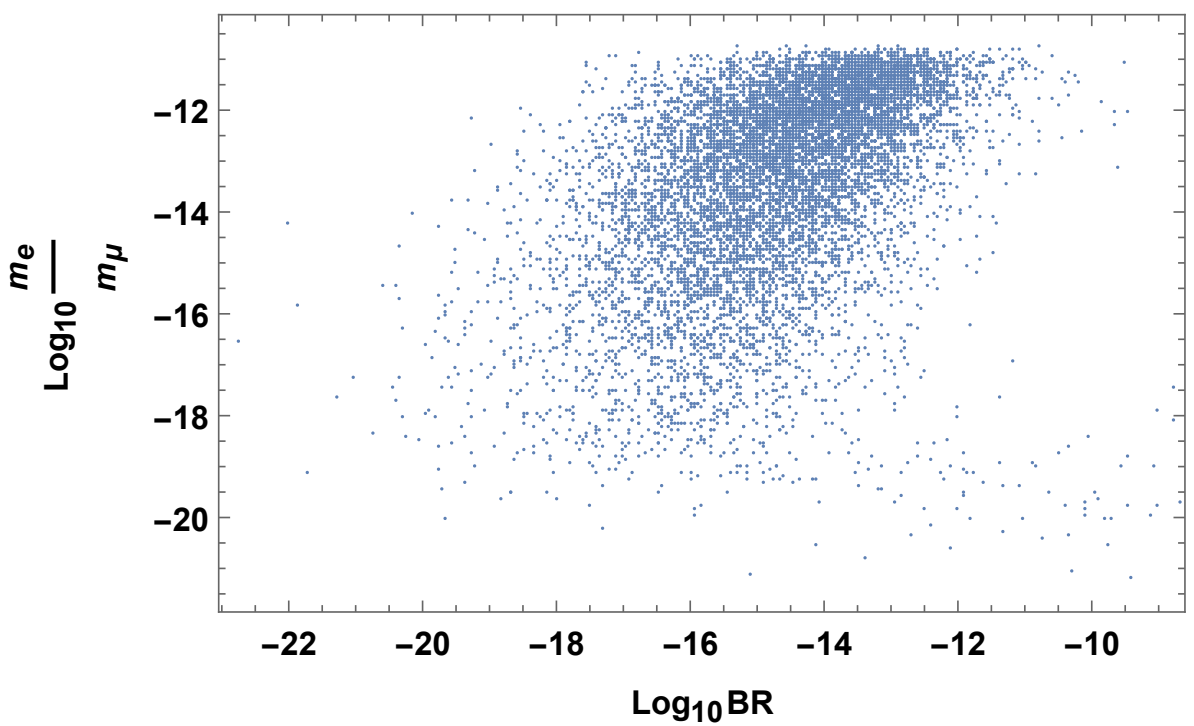

Figure 6.9: Correlation between $\frac{m_{e}}{m_{\mu}}$ and BR. Here one can see a small correlation between them. 


\section{Conclusion}

In view of all the results presented in this work, which have shown to be remarkably consistent with the experimental data, one can safely state that the Clockwork mechanism has proven to successfully reproduce all the hierarchical structure present in the fermion sector in a much more natural way than the SM, since it is very likely that the distributions for the Clockwork model, presented in Chapter 5, reproduce the observed hierarchies, whilst, as seen in Chapter 2, the distribution obtained via the SM configuration are extremely unlikely to reproduce the detected hierarchical spectra. In that sense, the Froggatt-Nielsen can also be regarded as more natural than the SM, as the latter has even produced distributions that fits a slightly better (lower value for $\chi^{2}$ ) than the Clockwork model. However, when comparing both of hierarchy models presented in this work, one can note that the distributions in the Clockwork model depend on fewer parameters than it is seen for the distributions in the Froggatt-Nielsen mechanism and, in that regard, the former can be considered to be simpler. The results obtained for the Clockwork simulations are summarized in Table 7.1 and Table 7.2.

Table 7.1: Summarized results for the quark sector best fit free parameters obtained through simulations.

\begin{tabular}{cc}
\hline Quantity & Value \\
\hline$N_{q}$ & 9 \\
$N_{u}$ & 9 \\
$N_{d}$ & 6 \\
\hline$c_{u}$ & 1.70 \\
$c_{d}$ & 0.03 \\
\hline$\chi_{\min }^{2}$ & 8.59 \\
\hline
\end{tabular}

It is also worth noting that another advantage of the Clockwork model over the Froggatt-Nielsen mechanism is that while the latter requires the use of higher dimension operators $(d>4)$ from the starting point, the former is exclusively build from renormalizable operators which makes it a UV-complete theory. 
Table 7.2: Summarized results for the lepton sector best fit free parameters obtained through simulations.

\begin{tabular}{cc}
\hline Quantity & Value \\
\hline$N_{l}$ & 2 \\
$N_{e}$ & 9 \\
$N_{\nu}$ & 0 \\
\hline$c_{e}$ & 0.02 \\
$m_{R}$ & $4.26 \times 10^{14} \mathrm{GeV}$ \\
\hline$\chi_{\min }^{2}$ & 1.86 \\
\hline
\end{tabular}

An important prediction made by the model is the existence of many new matter particles at the mass scale of at least around $100 \mathrm{TeV}$ which might be achievable by future generation of colliders over the next decades.

In conclusion, in view of all the points exposed across the present work, one can confidently say that the Clockwork mechanism provides a good solution to the fermion hierarchy problem and has shown to be a very interesting tool to build new theories in the context of BSM physics.

An interesting proposal for future works would be to look at how a extension to the Clockwork mechanism could work in the context of Grand Unified Theory (GUT). 


\section{Bibliography}

[1] HOOGEVEEN, F.. The standard model prediction for the electric dipole moment of the electron. Nuclear Physics B, 341(2):322 - 340, 1990.

[2] CHATRCHYAN, S.; OTHERS. Observation of a new boson at a mass of $125 \mathrm{GeV}$ with the CMS experiment at the LHC. Phys. Lett., B716:30-61, 2012.

[3] Bertone, G.; HOOPER, D. ; SILK, J.. Particle dark matter: Evidence, candidates and constraints. Phys. Rept., 405:279-390, 2005.

[4] ROVELLI, C.. Loop quantum gravity. Living Rev. Rel., 1:1, 1998.

[5] GEORGI, H.; QUINN, H. R. ; WEINBERG, S.. Hierarchy of Interactions in Unified Gauge Theories. Phys. Rev. Lett., 33:451-454, 1974.

[6] FROGgATT, C. D.; NIELSEN, H. B.. Hierarchy of Quark Masses, Cabibbo Angles and CP Violation. Nucl. Phys., B147:277-298, 1979.

[7] TANABASHI, M.; OTHERS. Review of Particle Physics. Phys. Rev., D98(3):030001, 2018.

[8] NELSON, P.. Naturalness in Theoretical Physics. Am. Sci., 73:60-67, 1985.

[9] 'T HOOFT, G.; ITZYKSON, C.; JAFFE, A.; LEHMANN, H.; MITTER, P. K.; SINGER, I. M. ; STORA, R.. Recent Developments in Gauge Theories. Proceedings, Nato Advanced Study Institute, Cargese, France, August 26 - September 8, 1979. NATO Sci. Ser. B, 59:pp.1438, 1980.

[10] CSÁKI, C.; TANEDO, P.. Beyond the Standard Model. In: Proceedings, 2013 European School of High-Energy Physics (ESHEP 2013): Paradfurdo, Hungary, June 5-18, 2013, p. 169-268, 2015.

[11] GIUDICE, G. F.. Naturally Speaking: The Naturalness Criterion and Physics at the LHC. p. 155-178, 2008. 
[12] GIUDICE, G. F.; MCCUllough, M.. A Clockwork Theory. JHEP, 02:036, 2017.

[13] CRAIG, N.; GARCIA GARCIA, I. ; SUTHERLAND, D.. Disassembling the Clockwork Mechanism. JHEP, 10:018, 2017.

[14] GIUDICE, G. F.; MCCULlOUGH, M.. Comment on "Disassembling the Clockwork Mechanism". 2017.

[15] VON GERSDORFF, G.. Natural Fermion Hierarchies from Random Yukawa Couplings. JHEP, 09:094, 2017.

[16] WILLENBROCK, S.. Symmetries of the standard model. In: Physics in $\mathrm{D}>=4$. Proceedings, Theoretical Advanced Study Institute in elementary particle physics, TASI 2004, Boulder, USA, June 6-July 2, 2004, p. 3-38, 2004.

[17] PESKIN, M. E.; SCHROEDER, D. V.. An Introduction to quantum field theory. Addison-Wesley, Reading, USA, 1995.

[18] SCHWARTZ, M. D.. Quantum Field Theory and the Standard Model. Cambridge University Press, 2014.

[19] GOLDSTONE, J.. Field Theories with Superconductor Solutions. Nuovo Cim., 19:154-164, 1961.

[20] ENGLERT, F.; BROUT, R.. Broken symmetry and the mass of gauge vector mesons. Phys. Rev. Lett., 13:321-323, Aug 1964.

[21] BROUT, R.; ENGLERT, F.. Spontaneous symmetry breaking in gauge theories: A Historical survey. In: High-energy physics. Proceedings, International Europhysics Conference, Jerusalem, Israel, August 19-25, 1997, p. 3-10, 1998.

[22] HIGGS, P. W.. Broken symmetries and the masses of gauge bosons. Phys. Rev. Lett., 13:508-509, Oct 1964.

[23] GURALNIK, G. S.; HAGEN, C. R. ; KIBBLE, T. W. B.. Global conservation laws and massless particles. Phys. Rev. Lett., 13:585-587, Nov 1964.

[24] GURALNIK, G. S.. The History of the Guralnik, Hagen and Kibble development of the Theory of Spontaneous Symmetry Breaking and Gauge Particles. Int. J. Mod. Phys., A24:2601-2627, 2009. 
[25] GLASHOW, S. L.. The renormalizability of vector meson interactions. Nucl. Phys., 10:107-117, 1959.

[26] WEINBERG, S.. A model of leptons. Phys. Rev. Lett., 19:1264-1266, Nov 1967.

[27] SALAM, A.; WARD, J. C.. Weak and electromagnetic interactions. II Nuovo Cimento (1955-1965), 11(4):568-577, Feb 1959.

[28] AAD, G.; OTHERS. Measurements of the Higgs boson production and decay rates and constraints on its couplings from a combined ATLAS and CMS analysis of the LHC pp collision data at $\sqrt{s}=7$ and $8 \mathrm{TeV}$. JHEP, 08:045, 2016.

[29] CABIBBO, N.. Unitary symmetry and leptonic decays. Phys. Rev. Lett., 10:531-533, Jun 1963.

[30] KOBAYASHI, M.; MASKAWA, T.. CP Violation in the Renormalizable Theory of Weak Interaction. Prog. Theor. Phys., 49:652-657, 1973.

[31] MINKOWSKI, P.. $\mu \rightarrow e \gamma$ at a rate of one out of 109 muon decays? Physics Letters B, 67(4):421 - 428, 1977.

[32] GELL-MANN, M.; RAMOND, P. ; SLANSKY, R.. Complex Spinors and Unified Theories. Conf. Proc., C790927:315-321, 1979.

[33] MOHAPATRA, R. N.; SENJANOVIĆ, G.. Neutrino mass and spontaneous parity nonconservation. Phys. Rev. Lett., 44:912-915, Apr 1980.

[34] YANAGIDA, T.. Horizontal gauge symmetry and masses of neutrinos. Conf. Proc., C7902131:95-99, 1979.

[35] KING, S. F.. Neutrino mass models. Rept. Prog. Phys., 67:107-158, 2004.

[36] ALTARELLI, G.; FERUGLIO, F.. Theoretical models of neutrino masses and mixings. Springer Tracts Mod. Phys., 190:169-207, 2003. [,169(2002)].

[37] PONTECORVO, B.. Inverse beta processes and nonconservation of lepton charge. Sov. Phys. JETP, 7:172-173, 1958. [Zh. Eksp. Teor. Fiz.34,247(1957)]. 
[38] MAKI, Z.; NAKAGAWA, M. ; SAKATA, S.. Remarks on the unified model of elementary particles. Prog. Theor. Phys., 28:870-880, 1962. [,34(1962)].

[39] HALL, L. J.; MURAYAMA, H. ; WEINER, N.. Neutrino mass anarchy. Phys. Rev. Lett., 84:2572-2575, 2000.

[40] MANOHAR, A. V.. Effective field theories. Lect. Notes Phys., 479:311362, 1997.

[41] KAPLAN, D. B.. Five lectures on effective field theory. 2005.

[42] BONNET, F.; HIRSCH, M.; OTA, T. ; WINTER, W.. Systematic study of the $d=5$ Weinberg operator at one-loop order. JHEP, 07:153, 2012.

[43] GRZADKOWSKI, B.; ISKRZYNSKI, M.; MISIAK, M. ; ROSIEK, J.. Dimension-Six Terms in the Standard Model Lagrangian. JHEP, 10:085, 2010.

[44] WEINBERG, S.. Baryon- and lepton-nonconserving processes. Phys. Rev. Lett., 43:1566-1570, Nov 1979.

[45] WILCZEK, F.; ZEE, A.. Operator analysis of nucleon decay. Phys. Rev. Lett., 43:1571-1573, Nov 1979.

[46] BRODSKY, S. J.; HOYER, P.. The $\bar{h}$ Expansion in Quantum Field Theory. Phys. Rev., D83:045026, 2011.

[47] MANOHAR, A.; GEORGI, H.. Chiral quarks and the non-relativistic quark model. Nuclear Physics B, 234(1):189 - 212, 1984.

[48] GIUDICE, G. F.; GROJEAN, C.; POMAROL, A. ; RATTAZZI, R.. The Strongly-Interacting Light Higgs. JHEP, 06:045, 2007.

[49] POMAROL, A.. Higgs Physics. In: Proceedings, 2014 European School of High-Energy Physics (ESHEP 2014): Garderen, The Netherlands, June 18 - July 01 2014, p. 59-77, 2016.

[50] BABU, K. S.. TASI Lectures on Flavor Physics. In: Proceedings of Theoretical Advanced Study Institute in Elementary Particle Physics on The dawn of the LHC era (TASI 2008): Boulder, USA, June 2-27, 2008, p. 49-123, 2010. 
[51] KAPLAN, D. E.; RATTAZZI, R.. Large field excursions and approximate discrete symmetries from a clockwork axion. Phys. Rev., D93(8):085007, 2016.

[52] $\mathrm{CHOI}, \mathrm{K}$.; IM, S. H.. Realizing the relaxion from multiple axions and its UV completion with high scale supersymmetry. JHEP, 01:149, 2016.

[53] AHMED, A.; DILLON, B. M.. Clockwork Goldstone Bosons. Phys. Rev., D96(11):115031, 2017.

[54] HONG, D. K.; KIM, D. H. ; SHIN, C. S.. Clockwork graviton contributions to muon $g-2$. Phys. Rev., D97(3):035014, 2018.

[55] PARK, S. C.; SHIN, C. S.. Clockwork seesaw mechanisms. Phys. Lett., B776:222-226, 2018.

[56] GIUDICE, G. F.; KATS, Y.; MCCULLOUGH, M.; TORRE, R. ; URBANO, A.. Clockwork/linear dilaton: structure and phenomenology. JHEP, 06:009, 2018.

[57] COY, R.; FRIGERIO, M. ; IBE, M.. Dynamical Clockwork Axions. JHEP, 10:002, 2017.

[58] KEHAGIAS, A.; RIOTTO, A.. Clockwork Inflation. Phys. Lett., B767:7380, 2017.

[59] LEE, H. M.. Gauged $U(1)$ clockwork theory. Phys. Lett., B778:79-87, 2018.

[60] KEHAGIAS, A.; RIOTTO, A.. The Clockwork Supergravity. JHEP, 02:160, 2018.

[61] IBANEZ, L. E.; MONTERO, M.. A Note on the WGC, Effective Field Theory and Clockwork within String Theory. JHEP, 02:057, 2018.

[62] KIM, J.; MCDONALD, J.. Freeze-In Dark Matter from a subHiggs Mass Clockwork Sector via the Higgs Portal. Phys. Rev., D98(12):123503, 2018.

[63] AGRAWAL, P.; FAN, J. ; REECE, M.. Clockwork Axions in Cosmology: Is Chromonatural Inflation Chrononatural? JHEP, 10:193, 2018.

[64] CHOI, K.; IM, S. H. ; SHIN, C. S.. General Continuum Clockwork. JHEP, 07:113, 2018. 
[65] TERESI, D.. Clockwork without supersymmetry. Phys. Lett., B783:16, 2018.

[66] NIEDERMANN, F.; PADILlA, A. ; SAFFIN, P. M.. Higher Order Clockwork Gravity. Phys. Rev., D98(10):104014, 2018.

[67] GOUDELIS, A.; MOHAN, K. A. ; SENGUPTA, D.. Clockworking FIMPs. JHEP, 10:014, 2018.

[68] ALONSO, R.; CARMONA, A.; DILLON, B. M.; KAMENIK, J. F.; MARTIN CAMALICH, J. ; ZUPAN, J.. A clockwork solution to the flavor puzzle. JHEP, 10:099, 2018.

[69] IBARRA, A.; KUSHWAHA, A. ; VEMPATI, S. K.. Clockwork for Neutrino Masses and Lepton Flavor Violation. Phys. Lett., B780:86-92, 2018.

[70] HABER, H. E.; KANE, G. L.. The Search for Supersymmetry: Probing Physics Beyond the Standard Model. Phys. Rept., 117:75263, 1985.

[71] LEE, T. D.. A Theory of Spontaneous T Violation. Phys. Rev., D8:1226-1239, 1973. [,516(1973)].

[72] PATEL, K. M.. Clockwork mechanism for flavor hierarchies. Phys. Rev., D96(11):115013, 2017.

[73] ARCHILLI, F.; BETTLER, M.-O.; OWEN, P. ; A. PETRIDIS, K.. Flavourchanging neutral currents making and breaking the standard model. Nature, 546:221-226, 062017.

[74] SIRUNYAN, A. M.; OTHERS. Search for the flavor-changing neutral current interactions of the top quark and the Higgs boson which decays into a pair of b quarks at $\sqrt{s}=13 \mathrm{TeV}$. JHEP, 06:102, 2018.

[75] ANDERSON, S.; OTHERS. Improved upper limits on the FCNC decays $B \rightarrow K \ell^{+} \ell^{-}$and $B \rightarrow \mathbf{K}^{*}(892) \ell^{+} \ell^{-}$. Phys. Rev. Lett., 87:181803, 2001.

[76] BARBERIO, E.; OTHERS. Averages of $b$-hadron and $c$-hadron Properties at the End of 2007. 2008.

[77] BECHER, T.; NEUBERT, M.. Analysis of $\mathrm{Br}($ anti-B $\rightarrow \mathrm{X}(\mathrm{s}$ gamma)) at NNLO with a cut on photon energy. Phys. Rev. Lett., 98:022003, 2007. 
[78] NISHI, C. C.. Simple derivation of general Fierz-like identities. Am. J. Phys., 73:1160-1163, 2005.

[79] Kinematics, cross-section formulae, and plots. Physics Letters B, 667(1):340 - 370, 2008. Review of Particle Physics. 